Key Words:

Sodium Bearing Waste

Steam Reforming

Durability Testing

THOR Treatment Technologies ${ }^{\circledR}$

Retention:

Permanent

\title{
DURABILITY TESTING OF FLUIDIZED BED STEAM REFORMER (FBSR) WASTE FORMS FOR SODIUM BEARING WASTE (SBW) AT IDAHO NATIONAL LABORATORY (INL)
}

\author{
Charles L. Crawford \\ Carol M. Jantzen
}

AUGUST 2007

Washington Savannah River Company
Savannah River Site
Aiken, SC 29808
$\begin{aligned} & \text { Prepared for the U.S. Department of Energy } \\ & \text { Under Contract Number DEAC09-96-SR18500 }\end{aligned}$


WSRC-STI-2007-00319, REV. 0

\section{DISCLAIMER}

This report was prepared for the United States Department of Energy under Contract No. DE-AC09-96SR18500 and is an account of work performed under that contract. Neither the United States Department of Energy, nor WSRC, nor any of their employees makes any warranty, expressed or implied, or assumes any legal liability or responsibility for accuracy, completeness, or usefulness, of any information, apparatus, or product or process disclosed herein or represents that its use will not infringe privately owned rights. Reference herein to any specific commercial product, process, or service by trade name, trademark, name, manufacturer or otherwise does not necessarily constitute or imply endorsement, recommendation, or favoring of same by Washington Savannah River Company or by the United States Government or any agency thereof. The views and opinions of the authors expressed herein do not necessarily state or reflect those of the United States Government or any agency thereof.

Printed in the United States of America

Prepared For

U.S. Department of Energy 
Key Words:

Sodium Bearing Waste

Steam Reforming

Durability Testing

THOR Treatment Technologies ${ }^{\circledR}$

Retention:

Permanent

DURABILITY TESTING OF FLUIDIZED BED STEAM REFORMER (FBSR) WASTE FORMS FOR SODIUM BEARING WASTE (SBW) AT IDAHO NATIONAL LABORATORY (INL)

\author{
Charles L. Crawford \\ Carol M. Jantzen
}

AUGUST 2007

\begin{tabular}{l} 
Washington Savannah River Company \\
Savannah River Site \\
Aiken, SC 29808 \\
\hline $\begin{array}{l}\text { Prepared for the U.S. Department of Energy } \\
\text { Under Contract Number DEAC09-96-SR18500 }\end{array}$
\end{tabular}




\section{REVIEWS AND APPROVALS}

\section{AUTHOR(S):}

Charles L. Crawford, Process Science and Engineering

Carol M. Jantzen, Process Science and Engineering

\section{TECHNICAL REVIEWER:}

John M. Pareizs, Process Science and Engineering

\section{APPROVERS:}

David A. Crowley, Process Science and Engineering

Date

Richard E. Edwards, Manager, Process Science and Engineering

Date
Date

Date 
WSRC-STI-2007-00319, REV. 0

\section{EXECUTIVE SUMMARY}

Fluidized Bed Steam Reforming (FBSR) processing of Sodium Bearing Waste simulants was performed in December 2006 by THOR $^{\mathrm{sm}}$ Treatment Technologies LLC (TTT) The testing was performed at the Hazen Research Inc. (HRI) pilot plant facilities in Golden, CO. FBSR products from these pilot tests on simulated waste representative of the SBW at the Idaho Nuclear Technology and Engineering Center (INTEC) were subsequently transferred to the Savannah River National Laboratory (SRNL) for characterization and leach testing. Four asreceived Denitration and Mineralization Reformer (DMR) granular/powder samples and four High Temperature Filter (HTF) powder samples were received by SRNL. FBSR DMR samples had been taken from the "active" bed, while the HTF samples were the fines collected as carryover from the DMR. The process operated at high fluidizing velocities during the mineralization test such that nearly all of the product collected was from the HTF. Active bed samples were collected from the DMR to monitor bed particle size distribution.

Characterization of these crystalline powder samples shows that they are primarily $\mathrm{Al}, \mathrm{Na}$ and $\mathrm{Si}$, with $>1 \mathrm{wt} \% \mathrm{Ca}, \mathrm{Fe}$ and $\mathrm{K}$. The DMR samples contained less than $1 \mathrm{wt} \%$ carbon and the HTF samples ranged from 13 to $26 \mathrm{wt} \%$ carbon. X-ray diffraction analyses show that the DMR samples contained significant quantities of the $\mathrm{Al}_{2} \mathrm{O}_{3}$ startup bed. The DMR samples became progressively lower in starting bed alumina with major $\mathrm{Na} / \mathrm{Al} / \mathrm{Si}$ crystalline phases (nepheline and sodium aluminosilicate) present as cumulative bed turnover occurred but $100 \%$ bed turnover was not achieved. The HTF samples also contained these major crystalline phases.

Durability testing of the DMR and HTF samples using the ASTM C1285 Product Consistency Test (PCT) 7-day leach test at $90^{\circ} \mathrm{C}$ was performed along with several reference glass samples. Comparison of the normalized leach rates for the various DMR and HTF components was made with the reference glasses and the Low Activity Waste (LAW) specification for the Hanford Waste Treatment and Vitrification Plant (WTP). Normalized releases from the DMR and HTF samples were all less than $1 \mathrm{~g} / \mathrm{m}^{2}$. For comparison, normalized release from the High-Level Waste (HLW) benchmark Environmental Assessment (EA) glass for $\mathrm{Si}, \mathrm{Li}, \mathrm{Na}$ and $\mathrm{B}$ ranges from 2 to $8 \mathrm{~g} / \mathrm{m}^{2}$. The normalized release specification for LAW glass for the Hanford WTP is $2 \mathrm{~g} / \mathrm{m}^{2}$.

The Toxicity Characteristic Leach Test (TCLP) was performed on DMR and HTF asreceived samples and the tests showed that these products meet the criteria for the EPA RCRA Universal Treatment Standards for all of the constituents contained in the starting simulants such as $\mathrm{Cr}, \mathrm{Pb}$ and $\mathrm{Hg}$ (RCRA characteristically hazardous metals) and $\mathrm{Ni}$ and $\mathrm{Zn}$ (RCRA metals required for listed wastes). 


\section{TABLE OF CONTENTS}

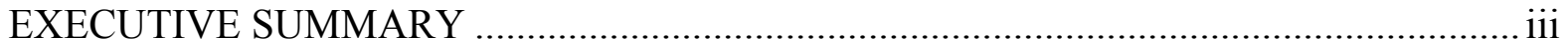

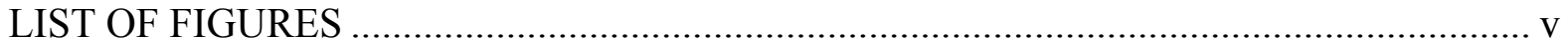

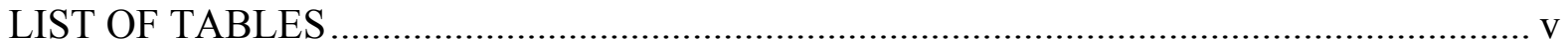

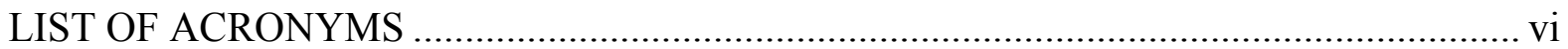

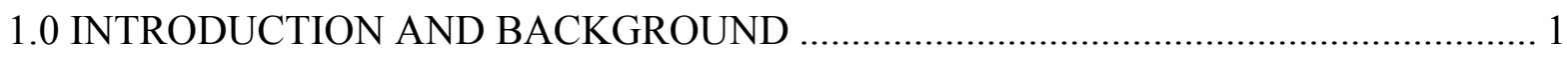

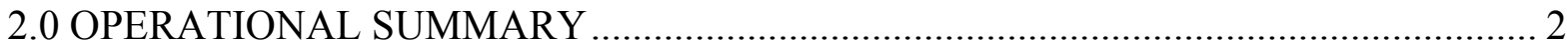

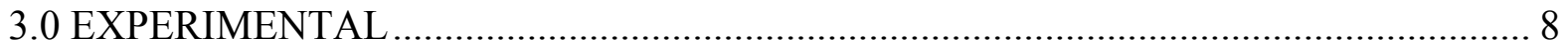

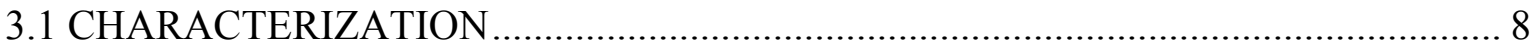

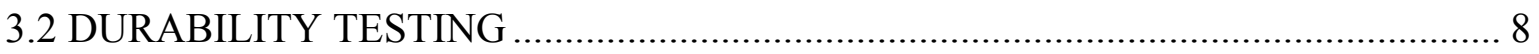

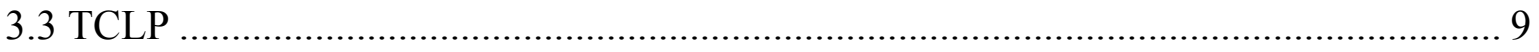

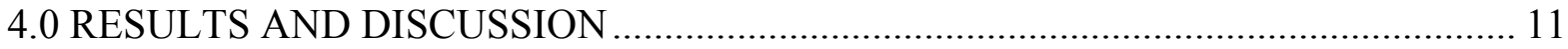

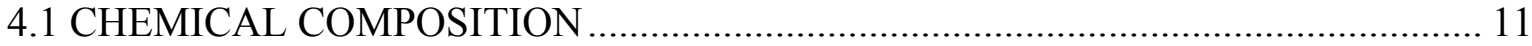

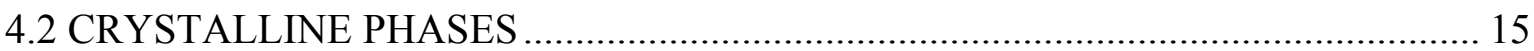

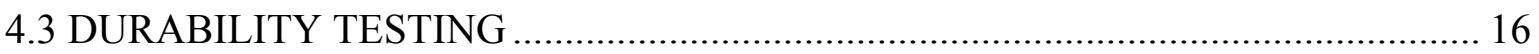

4.3.1 Comparison of Normalized Release to High-Level Waste ..................................... 22

4.3.2 Comparison of Normalized Release to Low Activity Waste ................................... 22

4.3.3 Comparison of 2007 FBSR Product Durability Testing to Previous Work ............ 25

4.4 TOXICITY CHARACTERISTIC LEACHING PROCEDURE ................................... 28

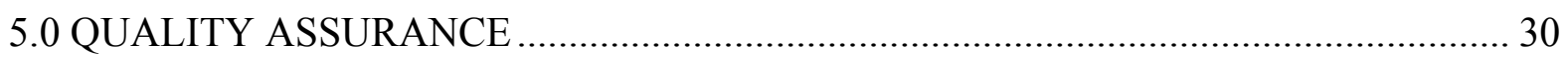

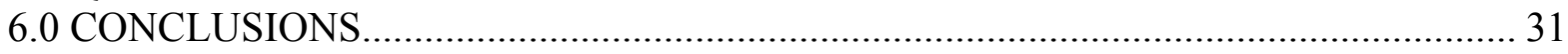

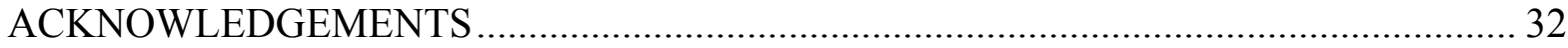

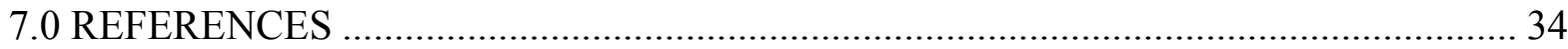

APPENDIX A - XRD SPECTRA OF DMR AND HTF SAMPLES ................................... 36

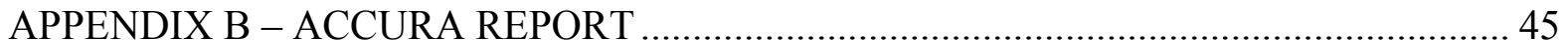




\section{LIST OF FIGURES}

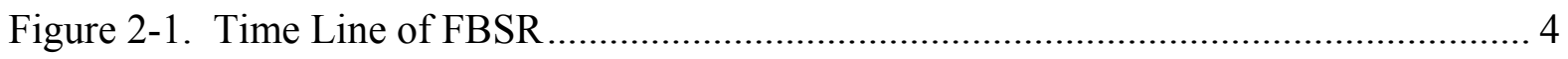

Figure 2-2. Ternary Plot Showing DMR and HTF Samples ............................................. 5

Figure 2-3. Ternary Plot Showing Adjusted DMR Compositions Relative to the Target

Compositions Defined by the Starting Simulant and the Starting Clay by Adjustment for

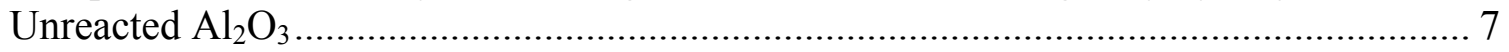

Figure 4-1. Normalized Release for DMR vs. EA Glass................................................... 23

Figure 4-2. Normalized Release for HTF vs. EA Glass ..................................................... 23

Figure 4-3. Normalized Release for DMR vs. LRM Glass and Hanford Contract LAW...... 24

Figure 4-4. Normalized Release for HTF vs. LRM Glass and Hanford Contract LAW ....... 24

Figure 4-5. Linearity of Alkali (NL(Na)) and Alumina Released to Solution........................ 25

Figure 4-6. Linearity of Alkali (NL(Cs)) and Alumina Released to Solution....................... 26

Figure 4-7. Release of Re, S, and Si to the PCT Leachates as a Function of the $\mathrm{pH}$ of the

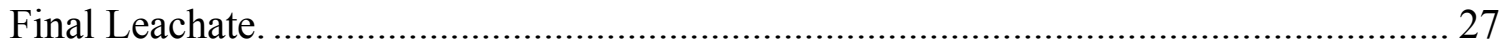

Figure 4-8. Relation of Re to $\mathrm{S}, \mathrm{Re}$ to $\mathrm{Si}$, and $\mathrm{S}$ to $\mathrm{Si}$ in the PCT Leachates of the FBSR

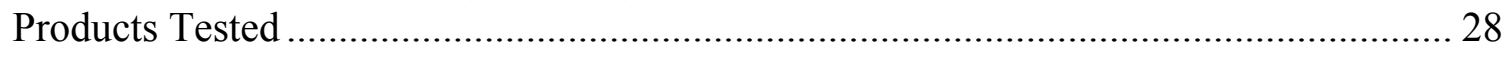

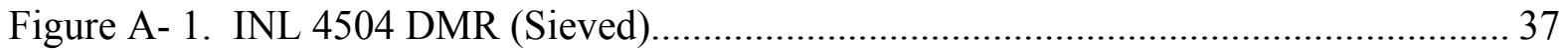

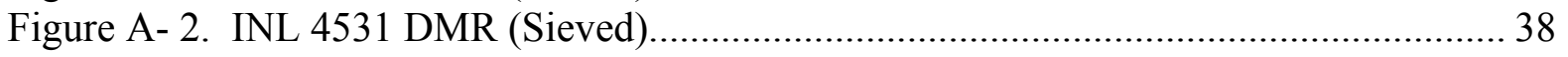

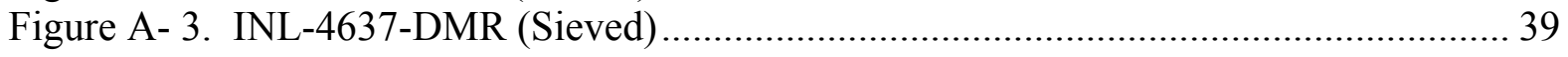

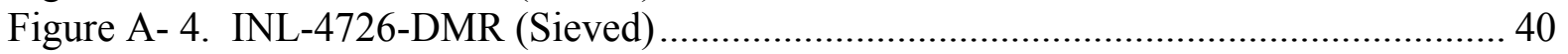

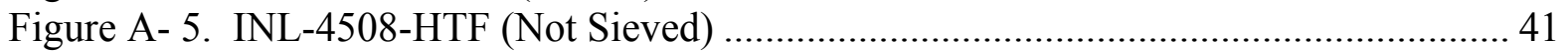

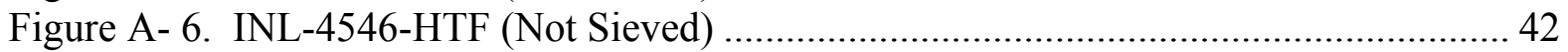

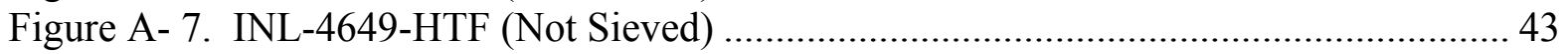

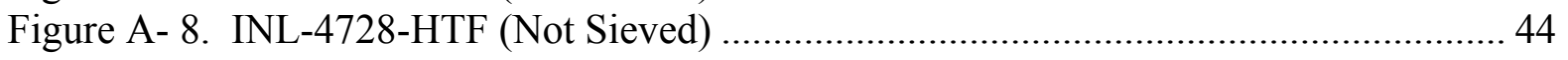

\section{LIST OF TABLES}

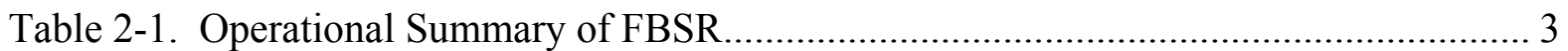

Table 2-2. MINCALC Results for DMR Adjustments for Unreacted $\mathrm{Al}_{2} \mathrm{O}_{3}$........................ 6

Table 4-1. Elemental Composition - Wt\% ……………................................................. 12

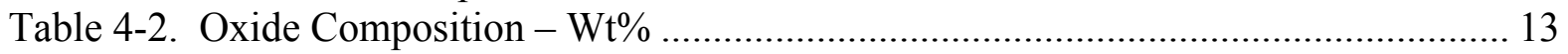

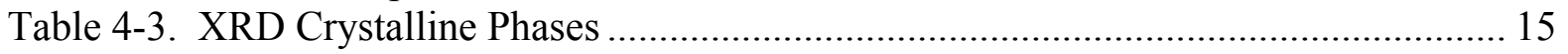

Table 4-4. BET and Geometric Surface Area Data ………............................................... 17

Table 4-5. PCT Leachate Standards and Blanks (ppm) ………............................................. 18

Table 4-6. PCT Leachate Standards and Blanks $(\mathrm{g} / \mathrm{L})$.......................................................... 19

Table 4-7. PCT Leachate Concentrations (ppm) ……….................................................. 20

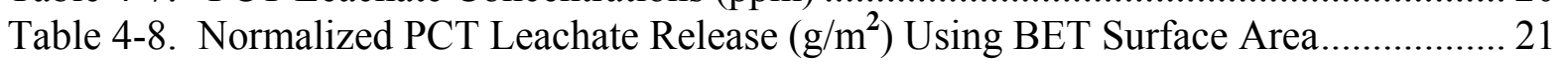

Table 4-9. Toxicity Characteristic Leaching Procedure (TCLP) Results ............................... 29 


\section{LIST OF ACRONYMS}

$\begin{array}{ll}\text { ARM-1 } & \text { Approved Reference Material - 1 } \\ \text { ASTM } & \text { American Society for Testing and Materials } \\ \text { DMR } & \text { Denitration and Mineralization Reformer } \\ \text { DOE } & \text { Department of Energy } \\ \text { EA } & \text { Environmental Assessment } \\ \text { EPA } & \text { Environmental Protection Agency } \\ \text { FBSR } & \text { Fluidized Bed Steam Reformer } \\ \text { HLW } & \text { High Level Waste } \\ \text { HRI } & \text { Hazen Research Inc. } \\ \text { HTF } & \text { High Temperature Filter } \\ \text { ICP-AES } & \text { Inductively Coupled Plasma - Atomic Emission Spectroscopy } \\ \text { ICP-MS } & \text { Inductively Coupled Plasma - Mass Spectroscopy } \\ \text { INTEC } & \text { Idaho Nuclear Technology and Engineering Center } \\ \text { LAW } & \text { Low Activity Waste } \\ \text { LRM } & \text { Low-activity Reference Material } \\ \text { NAS } & \text { Sodium Aluminosilicates } \\ \text { PCT } & \text { Product Consistency Testing } \\ \text { RCRA } & \text { Resource Conservation and Recovery Act } \\ \text { SA/V } & \text { Surface Area to Volume } \\ \text { SBW } & \text { Sodium Bearing Waste } \\ \text { SRNL } & \text { Savannah River National Laboratory } \\ \text { TCLP } & \text { Toxicity Characteristic Leaching Procedure } \\ \text { TRU } & \text { Transuranic } \\ \text { TTT } & \text { THOR } \\ \text { UTS Treatment Technologies } & \text { Universal Treatment Standards } \\ \text { WAPS } & \text { Waste Acceptance Product Specifications } \\ \text { WFO } & \text { Work for Others } \\ \text { WIPP } & \text { Waste Isolation Pilot Plant } \\ \text { XRD } & \text { X-ray Diffraction } \\ & \end{array}$




\subsection{INTRODUCTION AND BACKGROUND}

Fluidized Bed Steam Reforming (FBSR) has been selected by the United States Department of Energy (DOE) as the preferred treatment technology for the Sodium Bearing Waste (SBW) at the Idaho Nuclear Technology and Engineering Center (INTEC) [Fed. Register 2005]. The FBSR application for treatment of SBW simulants has been reported in previous studies [Mason 2006, Burket 2005, Soelberg 2004a, Soelberg 2004b]. Characterization and durability testing of FBSR bed and fines material produced at pilot scale facilities have been reported [Pareizs et al., 2005, Jantzen et al, 2006a, Jantzen et al, 2006b]. Durability testing of those materials in monolithic form was also studied [Jantzen 2006c].

Recent FBSR processing of simulated Idaho SBW was performed in December of 2006 at the Hazen Research Inc. (HRI) Facility in Golden, CO [Pilot Plant Report 2007]. Bed product materials and filter fines were produced and transferred to the Savannah River National Laboratory (SRNL) for characterization and durability testing as part of a Work for Others (WFO) project. This report presents the results of characterization, durability testing, and toxicity characteristic leaching procedure (TCLP) testing on these materials. 


\subsection{OPERATIONAL SUMMARY}

The HRI facility tests were performed December 12-21, 2006. Figure 2-1 and Table 2-1 show operational details that were provided by TTT to SRNL. A total of four as-received Denitration and Mineralization Reformer (DMR) samples and four High Temperature Filter (HTF) samples were received by SRNL.

The DMR samples were taken from the "active" bed, and HTF are the fines collected downstream of the DMR. The process operated at high fluidization during the mineralization test such that nearly all of the product collected was from the HTF. Active bed samples were collected from the DMR to monitor bed particle size distribution.

The major difference in the samples (and tests) is the quantity of clay that was combined with the liquid waste to "mineralize" the granular solid product that was produced in the DMR and HTF. Table 2-1 shows which samples are associated with the test condition (clay addition). The numerical value of the sample log numbers reflects the time line. The test was started with 228 , then 276 , then 339 , and finally $200 \mathrm{~g}$ clay/liter SBW.

A ternary plot showing the DMR and HTF 'as-received' sample compositions is shown in Figure 2-2. Note that as-received sample compositions will be presented in detail later in this report. This plot shows progression away from starting alumina bed turnover for the successive DMR samples. The earliest DMR sample 'DMR 4504' shown as the light green data point is nearest to the 'pure' $\mathrm{Al}_{2} \mathrm{O}_{3}$ point on the ternary diagram. As testing proceeded, successive DMR product sample compositions got further away from the $\mathrm{Al}_{2} \mathrm{O}_{3}$ portion of the ternary plot and closer to the targeted composition of the HTF samples.

One can use the software MINCALC process control strategy* to adjust the as-received DMR product compositions to account for the excess starting bed $\mathrm{Al}_{2} \mathrm{O}_{3}$. Table 2-2 shows the MINCALC results for calculated portions of starting bed $\mathrm{Al}_{2} \mathrm{O}_{3}$ and DMR product. These adjusted compositions can be normalized based on the ternary components $\left(\mathrm{Al}_{2} \mathrm{O}_{3}, \mathrm{M}_{2} \mathrm{O}=\mathrm{Na}_{2} \mathrm{O}\right.$ $+\mathrm{K}_{2} \mathrm{O}+\mathrm{Cs}_{2} \mathrm{O}$, and $\mathrm{SiO}_{2}$ ), and plotted on the ternary plot shown in Figure 2-3. Figure 2-3 also shows the $\mathrm{Na}_{2} \mathrm{O} / \mathrm{Al}_{2} \mathrm{O}_{3}$ starting point for the $\mathrm{SBW}$ simulant as well as the $\mathrm{SiO}_{2} / \mathrm{Al}_{2} \mathrm{O}_{3}$ starting point for the clay. Comparison of the adjusted DMR data shown on Figure 2-3 ternary indicates that the product portion of the as-received DMR samples are indeed close to the target composition as defined by the line connecting the SWB simulant and the clay.

\footnotetext{
* MINCALC is a spreadsheet that aids in Steam Reforming batch preparations (clay and waste amounts, carbon additions, etc.) by predicting product mineral phases. Further details of MINCALC have been previously presented in Pareizs et al., 2005.
} 
Table 2-1. Operational Summary of FBSR

\begin{tabular}{|c|c|c|c|l|}
\hline Sample Log \# & Date & Time & Location & Test Condition \\
\hline 4504 & $12 / 15 / 06$ & 0115 & DMR & $\begin{array}{l}\text { Production Tests P-1 \& P-2 } \\
(228 \text { g clay / L SBW Lite })\end{array}$ \\
\hline 4508 & $12 / 15 / 06$ & 0115,0145 & HTF & (same as above) \\
\hline 4531 & $12 / 16 / 06$ & 0015 & DMR & $\begin{array}{l}\text { Production Tests P-3 } \\
(276 \text { g clay / L SBW Lite })\end{array}$ \\
\hline 4546 & $12 / 16 / 06$ & $0215-0245$ & HTF & (same as above) \\
\hline 4637 & $12 / 18 / 06$ & 0100 & DMR & $\begin{array}{l}\text { Production Tests P-4 } \\
(339 \text { g clay / L SBW Lite })\end{array}$ \\
\hline 4649 & $12 / 18 / 06$ & $0100-0140$ & HTF & (same as above) \\
\hline 4726 & $12 / 20 / 06$ & 0100 & DMR & $\begin{array}{l}\text { Production Tests P-5A } \\
(200 \text { g clay / L SBW Lite })\end{array}$ \\
\hline 4728 & $12 / 20 / 06$ & 0030 & HTF & (same as above) \\
\hline
\end{tabular}




\section{ESTD Mineralizing (Production) Time Line}

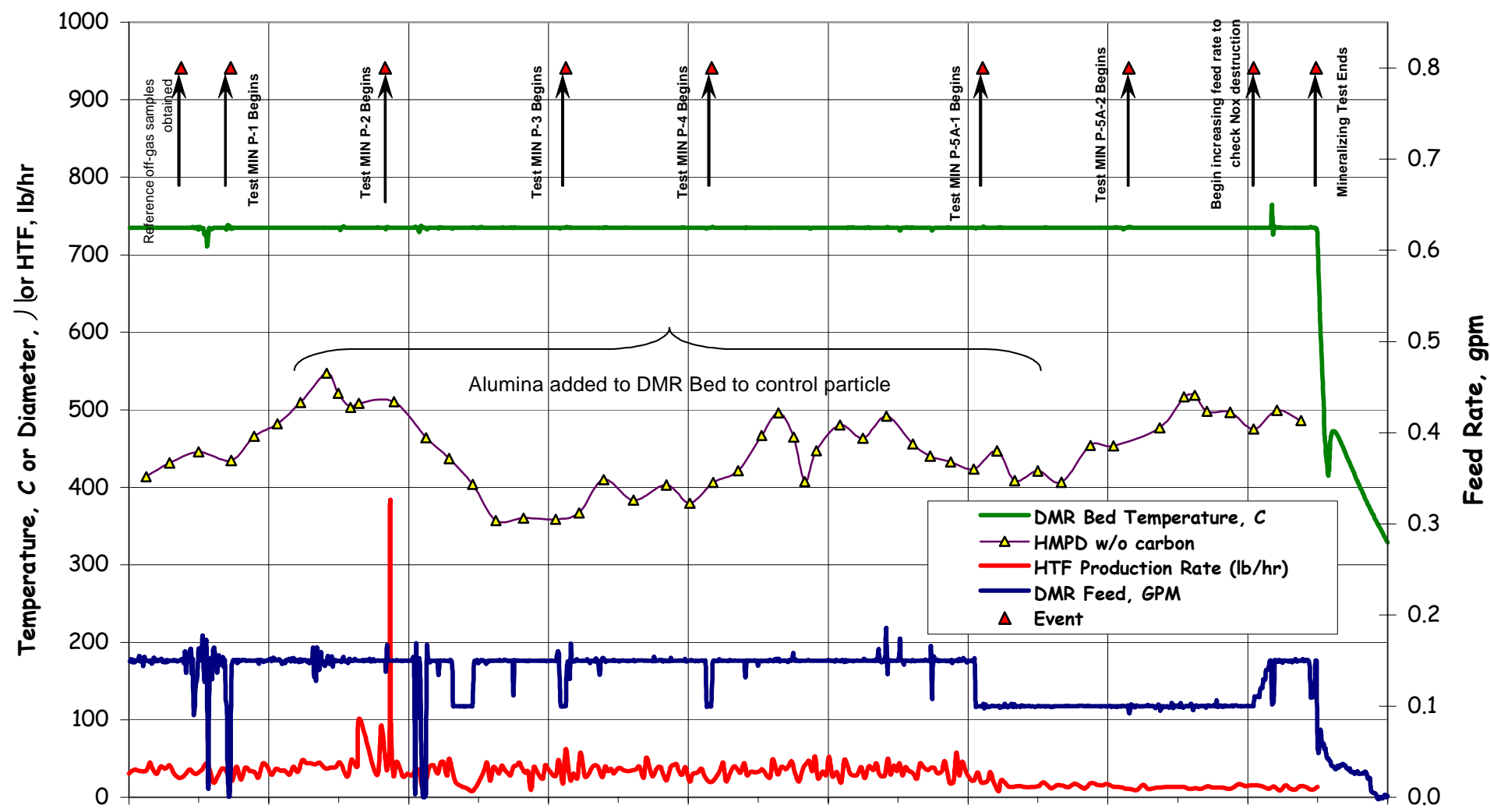

12/12/06 0:00 12/13/06 0:00 12/14/06 0:00 12/15/06 0:00 12/16/06 0:00 12/17/06 0:00 12/18/06 0:00 12/19/06 0:00 12/20/06 0:00 12/21/06 0:00

Date/Time

Figure 2-1. Time Line of FBSR 


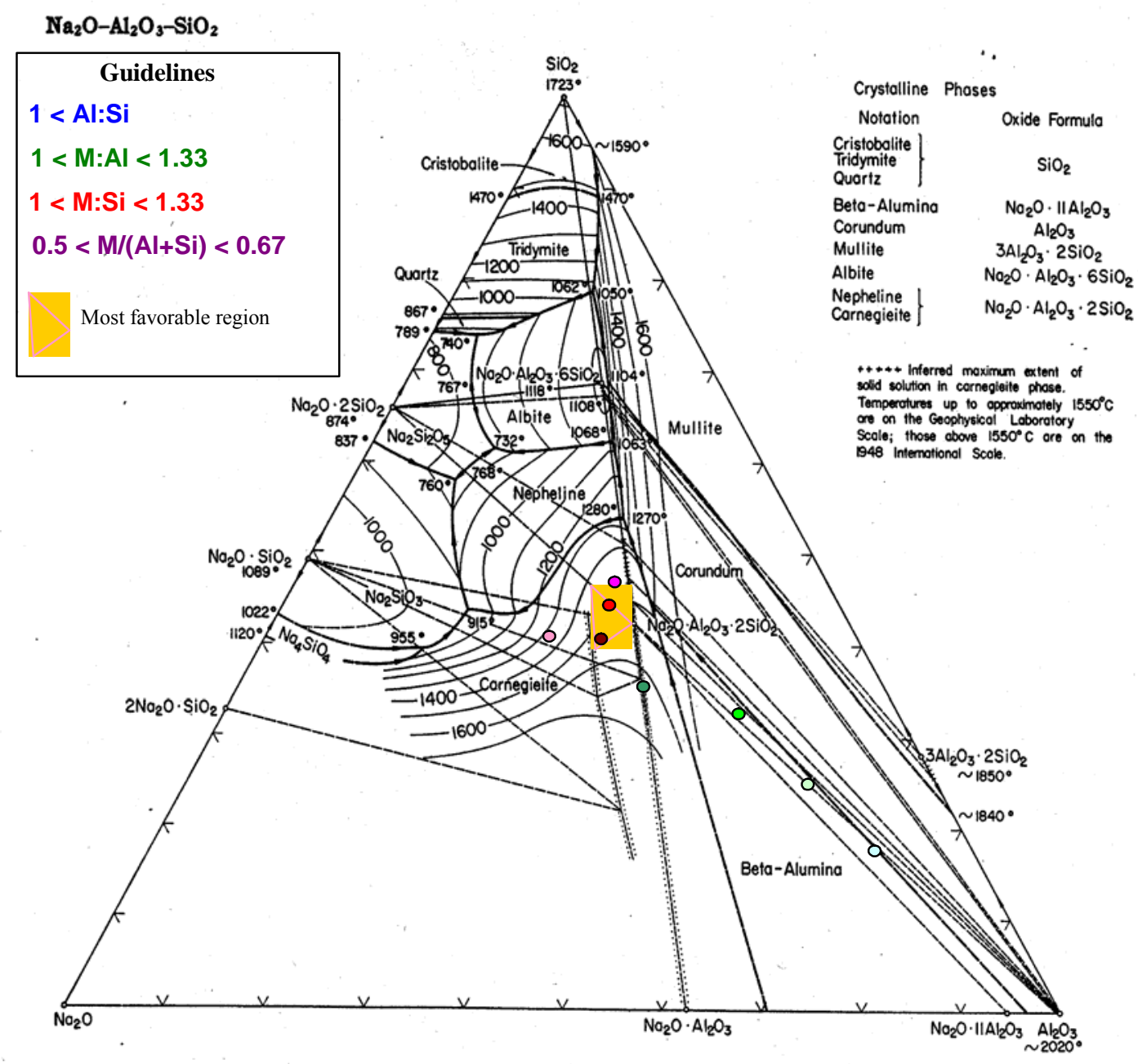

FIG. 501.-System $\mathrm{Na}_{2} \mathrm{O}-\mathrm{Al}_{2} \mathrm{O}_{8}-\mathrm{SiO}_{2}$; composite.

E. F. Osborn and Arnulf Muan, revised and redrawn "Phase Equilibrium Diagrams of Oxide Systems," Plate 4, published by the American Ceramic Society and the Edward Orton, Jr., Ceramic Foundation, 1960.

\begin{tabular}{rrrrrr}
\multicolumn{2}{r}{ DMR Samples } & g clay I L & \multicolumn{2}{c}{ HTF Samples } & g clay I L \\
$\bigcirc$ & 4504 & 228 & $\bigcirc$ & 4508 & 228 \\
$\bigcirc$ & 4531 & 276 & $\bigcirc$ & 4546 & 276 \\
$\bigcirc$ & 4637 & 339 & $\bigcirc$ & 4649 & 339 \\
$\bigcirc$ & 4726 & 200 & $\bigcirc$ & 4728 & 200
\end{tabular}

Figure 2-2. Ternary Plot Showing DMR and HTF Samples 
WSRC-STI-2007-00319, REV. 0

Table 2-2. MINCALC Results for DMR Adjustments for Unreacted $\mathrm{Al}_{2} \mathrm{O}_{3}$

\begin{tabular}{|c|c|c|c|c|c|c|}
\hline $\begin{array}{c}\text { DMR Sample } \\
\#\end{array}$ & Date & Time & & $\begin{array}{c}\text { As } \\
\text { Received }\end{array}$ & $\begin{array}{c}\text { Adjusted } \\
\text { for } \\
\text { Unreacted } \\
\mathrm{Al}_{2} \mathrm{O}_{3} \\
\end{array}$ & $\begin{array}{c}\text { Percentage Starting Bed } \\
\text { vs. Product }\end{array}$ \\
\hline 4504 & $12 / 15 / 06$ & 0115 & $\begin{array}{l}\mathrm{SiO}_{2} \\
\mathrm{Alkali} \\
\mathrm{Al}_{2} \mathrm{O}_{3} \\
\mathrm{Sum}\end{array}$ & $\begin{array}{c}17.30 \\
9.85 \\
72.85 \\
100.00\end{array}$ & $\begin{array}{c}43.17 \\
24.57 \\
32.26 \\
100.00\end{array}$ & $\begin{array}{c}60 \% \text { Starting Bed vs. } \\
40 \% \text { Product }\end{array}$ \\
\hline 4531 & $12 / 16 / 06$ & 0015 & $\begin{array}{l}\mathrm{SiO}_{2} \\
\mathrm{Alkali} \\
\mathrm{Al}_{2} \mathrm{O}_{3} \\
\mathrm{Sum}\end{array}$ & $\begin{array}{c}24.57 \\
12.68 \\
62.74 \\
100.00 \\
\end{array}$ & $\begin{array}{c}44.13 \\
22.77 \\
33.10 \\
100.00 \\
\end{array}$ & $\begin{array}{l}44 \% \text { Starting Bed vs. } \\
56 \% \text { Product }\end{array}$ \\
\hline 4637 & $12 / 18 / 06$ & 0100 & $\begin{array}{l}\mathrm{SiO}_{2} \\
\mathrm{Alkali} \\
\mathrm{Al}_{2} \mathrm{O}_{3} \\
\mathrm{Sum} \\
\end{array}$ & $\begin{array}{c}32.35 \\
15.83 \\
51.82 \\
100.00 \\
\end{array}$ & $\begin{array}{c}44.72 \\
21.88 \\
33.40 \\
100.00 \\
\end{array}$ & $\begin{array}{l}28 \% \text { Starting Bed vs. } \\
72 \% \text { Product }\end{array}$ \\
\hline 4726 & $12 / 20 / 06$ & 0100 & $\begin{array}{l}\mathrm{SiO}_{2} \\
\mathrm{Alkali} \\
\mathrm{Al}_{2} \mathrm{O}_{3} \\
\text { Sum }\end{array}$ & $\begin{array}{c}35.55 \\
23.87 \\
40.57 \\
100.00 \\
\end{array}$ & $\begin{array}{c}36.93 \\
24.80 \\
38.27 \\
100.00 \\
\end{array}$ & $\begin{array}{l}\text { 4\% Starting Bed vs. } \\
\text { 96\% Product }\end{array}$ \\
\hline
\end{tabular}


WSRC-STI-2007-00319, REV. 0

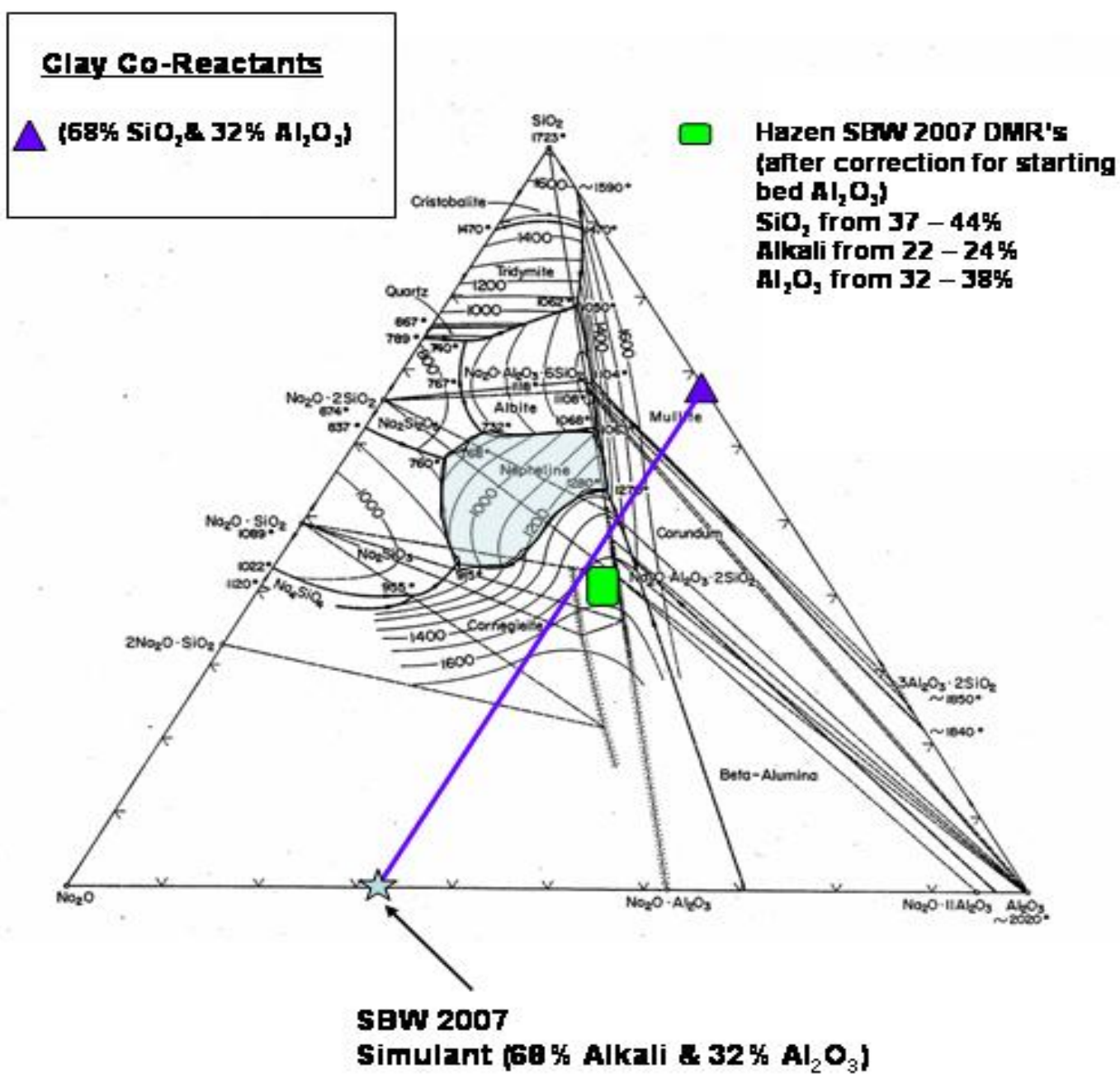

Figure 2-3. Ternary Plot Showing Adjusted DMR Compositions Relative to the Target Compositions Defined by the Starting Simulant and the Starting Clay by Adjustment for Unreacted $\mathrm{Al}_{2} \mathrm{O}_{3}$ 
WSRC-STI-2007-00319, REV. 0

\subsection{EXPERIMENTAL}

\subsection{CHARACTERIZATION}

Elemental and anion compositions of the steam reforming materials were measured for as received samples before and after heating of the samples at $525^{\circ} \mathrm{C}$ overnight. Elemental and anion analyses were performed on lithium tetraborate fusion $\left(1000^{\circ} \mathrm{C}\right)$ and sodium peroxide digestion $\left(650{ }^{\circ} \mathrm{C}\right)$, respectively. These methods used nominally $0.1 \mathrm{~g}$ of powder solid sample to $0.1 \mathrm{~L}$ of dissolved solution and have been described in detail previously (Pareizs et al., 2005). The digestion methods for elemental analysis involves the use of acids for dissolution. Water is used in place of acid in the sodium peroxide digestion for anion analysis. All elemental concentrations (except for $\mathrm{Hg}, \mathrm{Re}$ and $\mathrm{Cs}$ ) were determined by Inductively Coupled Plasma Atomic Emission Spectroscopy (ICP-AES). The Re and Cs were measured by Inductively Coupled Plasma Mass Spectroscopy (ICP-MS). Mercury was measured by performing a separate microwave dissolution, followed by cold vapor atomic absorption spectroscopy. REDOX (iron (II) to total iron ratio) was determined on samples that were not subjected to carbon removal, using a dissolution and absorption spectroscopy method. As received samples were also examined by powder X-ray Diffraction (XRD) to investigate the formation of the mineral phases in the FBSR waste forms.

\subsection{DURABILITY TESTING}

The chemical durability of the steam reformer products was determined using the Product Consistency Test (PCT) ASTM procedure C 1285-02 [ASTM 2002]. Prior to sizing and washing, carbon was removed from the material by heating overnight at $525^{\circ} \mathrm{C}$. The DMR product samples were sized between (-) 100 and (+) 200 mesh $(74 \mu \mathrm{m}$ to $149 \mu \mathrm{m})$, which is the same size fraction used to express glass waste form performance. The HTF fines material was sieved to (-) 200 mesh. The sized material was washed six times with $100 \%$ ethanol to remove electrostatic fines. Water was not used for washing so no potential water soluble phases would be removed prior to leaching as cautioned by the ASTM C1285-02 procedure. Portions of the washed and dried DMR and HTF powders were analyzed using Microtrac - S3000 instrumentation for particle size analysis by laser light scattering. BET surface area measurements via gas adsorption, and nitrogen gas pycnometry density measurements (Quantachrome Corp.) were also performed on the sieved/washed/dried portions of the powders used for PCT. For all samples, ASTM Type I water was used as leachant, a constant leachant to sample ratio of $10 \mathrm{~cm}^{3} / \mathrm{g}$ was used, the test temperature was $90^{\circ} \mathrm{C}$, and the test duration was seven days. Test duration and temperature are the nominal test conditions used for testing glass waste form performance under the PCT-A [ASTM 2002].

The PCT results can be expressed as a normalized concentration $\left(\mathrm{NC}_{\mathrm{i}}\right)$ which has units of $\mathrm{g}$ waste form $/ \mathrm{L}_{\text {leachant}}$, or as a normalized release $\left(\mathrm{NL}_{\mathrm{i}}\right)$ in $\mathrm{g}$ waste form $/ \mathrm{m}^{2}$. Normalized concentrations are calculated using Equation 1 and normalized release is calculated using Equation 2.

$\mathrm{NC}_{\mathrm{i}}=\mathrm{C}_{\mathrm{i}}($ sample $) / f_{\mathrm{i}}$

(Equation 1) 
Where $\quad \mathrm{C}_{\mathrm{i}}$ (sample) is the measured leachate concentration

$f_{\mathrm{i}}$ is the elemental weight fraction

$\mathrm{SA} / \mathrm{V}$ is the surface area to volume ratio

In order to calculate $\mathrm{NL}_{\mathrm{i}}$, the units used to express LAW glass durability, the surface area of the material being tested must either be calculated per ASTM C 1285, Appendix X1, or measured. In this study the SA/V was calculated using the average particle size diameter as determined from the geometric surface area and the powder bulk density via Equation 3.
$\mathrm{SA} / \mathrm{V}_{\text {calc }}=6 /(\rho \cdot \mathrm{d} \cdot \mathrm{V})$
(Equation 3)

Where $\quad \mathrm{SA} / \mathrm{V}_{\text {calc }}$ is the calculated surface area to volume ratio based on the average particle diameter and the waste form powder density

$\mathrm{d}$ is the average particle diameter $(\mathrm{m})$

$\rho$ is the waste form particle density $\left(\mathrm{g} / \mathrm{m}^{3}\right)$

$\mathrm{V}$ is the volume of leachant $\mathrm{V}$ per $\mathrm{g}$ of waste form $(\mathrm{L} / \mathrm{g})$

The other method for SA/V determination involves a measurement of the surface area by the BET method. In this method, the amount of an inert gas that condenses on a powdered sample is measured at a temperature near the boiling point of the gas. The amount of gas condensed on the sample is measured by the pressure change in the system upon exposure to the sample. This method measures all open pores, inclusions, irregularities, etc. that are penetrable by the inert gas. The $\mathrm{SA} / \mathrm{V}$ ratio is calculated by dividing the measured BET surface area by the leachant volume via Equation 4.

$\mathrm{SA} / \mathrm{V}_{\mathrm{BET}}=\mathrm{SA}_{\mathrm{BET}} / \mathrm{V}$

(Equation 4)

\subsection{TCLP}

The INL SBW is a listed waste under the EPA Resource Conservation and Recovery Act (RCRA). When treated, the waste form must retain the hazardous components at the Universal Treatment Standard (UTS) limits [Land Disposal Restrictions 2004]. The Land Disposal Restrictions (LDRs) do not apply to Transuranic (TRU) waste forms disposed of at the Waste Isolation Pilot Plant (WIPP). However, if a mineralized waste form were to be disposed of at the Federal Repository (such as Yucca Mountain), then LDRs (UTS) would apply.

All DMR and HTF samples were evaluated for retention of the hazardous metals by the EPA Toxicity Characteristic Leaching Procedure, Method 1311 (TCLP) [TCLP Method 1311, 1986]. Greater than $100 \mathrm{~g}$ samples of as-received material were submitted to Accura Analytical Laboratory, Inc. of Norcross, GA, an EPA-certified laboratory. In the leaching procedure, $100 \mathrm{~g}$ samples are extracted by an acidic fluid for 18 hours. The extraction fluid (leachate) is then filtered and analyzed for elements of interest. Since organics are destroyed in the FBSR process, only the following RCRA hazardous inorganic species were measured: $\mathrm{As}, \mathrm{Ba}, \mathrm{Cd}, \mathrm{Cr}, \mathrm{Pb}, \mathrm{Se}$, 
$\mathrm{Ag}, \mathrm{Hg}, \mathrm{Ni}$, and $\mathrm{Zn}$ It should be noted that although all of these constituents were analyzed for in the TCLP, the elements As, Ba, Cd, Se and Ag were not added to the SBW simulant that was processed. If the concentration of a hazardous inorganic species from the simulated waste form is higher than the UTS limits, then it is assumed that a real waste treated in a similar manner would fail the UTS limits and require further remediation. 


\subsection{RESULTS AND DISCUSSION}

\subsection{CHEMICAL COMPOSITION}

Tables 4-1 and 4-2 show wt\% elemental and oxide species, respectively, for the DMR and HTF samples. Data was obtained for both 'before ashing' samples (indicated with '-B' labels in Tables 4-1 and 4-2) and for samples that had been ashed at $525^{\circ} \mathrm{C}$ for $\sim 8 \mathrm{hrs}$ until no further mass change (indicated with '-A' labels in Tables 4-1 and 4-2). Boron and fluoride were added to the SBW simulants at $\sim 0.2 \mathrm{~g} / \mathrm{L}$ and $0.6 \mathrm{~g} / \mathrm{L}$ but were not detected in the dissolution/analysis of the DMR or HTF samples. Zirconium and cerium were also added to the SBW but were not analyzed in this characterization. Table 4-1 data indicates that no detectable mercury was present in any of the samples above the instrument detection limit of $0.004 \mathrm{wt} \%$. Elemental analysis of the samples shows that $\mathrm{Al}>\mathrm{Si} \sim \mathrm{Na}$ are the main components, with greater than $1 \mathrm{wt} \%$ of $\mathrm{Ca}, \mathrm{Fe}$ and $\mathrm{K}$ also present. Table 4-2 shows that no detectable nitrate anions were present in the DMR and HTF samples, and that some samples showed detectable nitrite levels with the HTF samples having $>1 \mathrm{wt} \% \mathrm{NO}_{2}{ }^{-}$. The carbon content of the HTF samples was in the range of $13-26 \mathrm{wt} \%$ and the DMR samples contained $<1 \mathrm{wt} \%$ carbon. All of the iron determined in the HTF samples was in the reduced ferrous $\left(\mathrm{Fe}^{2+}\right)$ state, as indicated by the REDOX values of $\left(\mathrm{Fe}^{2+} / \mathrm{Fe}\right.$ total) equal to one. Summation of all species on an oxide basis including the carbon content gives totals in an acceptable range of $96-104 \mathrm{wt} \%$. 
WSRC-STI-2007-00319, REV. 0

Table 4-1. Elemental Composition - Wt\%

\begin{tabular}{|c|c|c|c|c|c|c|c|c|c|c|c|c|c|c|c|c|c|c|c|c|c|c|c|c|c|}
\hline Sample ID & Al & $B^{*}$ & $\mathrm{Ca}$ & & $\mathrm{Cr}$ & $\mathrm{Fe}$ & $\mathrm{K}$ & $\mathrm{Mg}$ & $\mathrm{Mn}$ & $\mathrm{Na}$ & & $\mathrm{Ni}$ & $\mathbf{P}$ & & $\mathrm{Pb}$ & & $\mathrm{s}$ & $\mathrm{Si}$ & & $\mathrm{Sr}$ & $\mathrm{Ti}$ & $\mathrm{Zn}$ & Cs & $\operatorname{Re}$ & $\mathrm{Hg}^{* *}$ \\
\hline 4504-DMR-B & 35.35 & 0.1 & 1.83 & & 0.04 & 3.43 & 1.05 & 0.16 & 0.14 & 4.11 & & 0.02 & 0.07 & & 0.01 & $<$ & 0.03 & 5.14 & & 0.02 & 0.25 & 0.09 & $6.1 \mathrm{E}-03$ & $1.2 \mathrm{E}-03$ & 0.004 \\
\hline 4504-DMR-A & 33.70 & 0.1 & 2.45 & & 0.05 & 2.91 & 1.41 & 0.18 & 0.17 & 5.16 & & 0.02 & 0.08 & & 0.01 & & 0.04 & 7.11 & $<$ & 0.01 & 0.31 & 0.11 & $6.1 \mathrm{E}-03$ & $1.4 \mathrm{E}-03$ & NA \\
\hline 4531-DMR-B & 32.30 & 0.1 & 2.70 & & 0.05 & 4.07 & 1.54 & 0.20 & 0.19 & 5.10 & & 0.02 & 0.06 & & 0.01 & $<$ & 0.03 & 7.48 & & 0.02 & 0.38 & 0.14 & 7.1E-03 & $1.6 \mathrm{E}-03$ & 0.004 \\
\hline 4531-DMR-A & 28.85 & 0.1 & 3.26 & & 0.06 & 3.52 & 2.19 & 0.22 & 0.22 & 6.11 & & 0.03 & 0.09 & & 0.02 & & 0.04 & 9.68 & $<$ & 0.01 & 0.43 & 0.16 & $6.3 \mathrm{E}-03$ & $1.7 \mathrm{E}-03$ & NA \\
\hline 4637-DMR-B & 22.70 & 0.1 & 3.42 & & 0.06 & 2.93 & 2.53 & 0.25 & 0.22 & 8.42 & & 0.03 & 0.05 & & 0.02 & $<$ & 0.03 & 13.70 & & 0.02 & 0.65 & 0.19 & $1.0 \mathrm{E}-02$ & $3.1 \mathrm{E}-03$ & 0.004 \\
\hline 4637-DMR-A & 24.30 & 0.1 & 3.25 & & 0.06 & 3.15 & 2.46 & 0.23 & 0.21 & 8.31 & & 0.05 & 0.04 & & 0.02 & $<$ & 0.03 & 13.40 & & 0.01 & 0.57 & 0.19 & $1.1 \mathrm{E}-02$ & $1.6 \mathrm{E}-03$ & NA \\
\hline 4726-DMR-B & 19.35 & 0.1 & 4.26 & & 0.05 & 3.20 & 3.37 & 0.35 & 0.33 & 12.65 & & 0.02 & 0.08 & $<$ & 0.01 & & 0.04 & 14.85 & & 0.02 & 0.67 & 0.06 & $4.0 \mathrm{E}-03$ & $8.0 \mathrm{E}-04$ & 0.004 \\
\hline 4726-DMR-A & 19.25 & 0.1 & 4.78 & & 0.05 & 2.36 & 3.37 & 0.40 & 0.35 & 12.90 & & 0.02 & 0.08 & $<$ & 0.01 & & 0.04 & 15.00 & & 0.02 & 0.72 & 0.07 & $2.6 \mathrm{E}-03$ & $9.5 \mathrm{E}-04$ & NA \\
\hline 4508-HTF-B & 13.35 & 0.1 & 5.17 & & 0.06 & 1.34 & 2.99 & 0.29 & 0.24 & 9.86 & & 0.03 & 0.08 & & 0.03 & & 0.68 & 13.65 & $<$ & 0.01 & 0.65 & 0.10 & $9.3 \mathrm{E}-02$ & $6.8 \mathrm{E}-03$ & 0.004 \\
\hline 4508-HTF-A & 15.00 & 0.1 & 6.33 & & 0.07 & 1.66 & 3.82 & 0.32 & 0.28 & 11.85 & & 0.04 & 0.11 & & 0.04 & & 0.70 & 16.05 & & 0.02 & 0.77 & 0.10 & 1.1E-01 & $1.5 \mathrm{E}-02$ & NA \\
\hline 4546-HTF-B & 11.60 & 0.1 & 5.11 & & 0.05 & 1.14 & 3.01 & 0.26 & 0.22 & 8.77 & & 0.03 & 0.08 & & 0.03 & & 0.54 & 14.30 & & 0.01 & 0.67 & 0.07 & $9.1 \mathrm{E}-02$ & $6.3 \mathrm{E}-03$ & 0.004 \\
\hline 4546-HTF-A & 14.90 & 0.1 & 6.14 & & 0.07 & 1.50 & 3.41 & 0.32 & 0.28 & 10.90 & & 0.03 & 0.10 & & 0.05 & & 0.68 & 18.10 & & 0.02 & 0.83 & 0.10 & 1.0E-01 & 1.8E-02 & NA \\
\hline 4649-HTF-B & 10.30 & 0.1 & 6.36 & & 0.04 & 0.61 & 2.41 & 0.27 & 0.20 & 6.99 & & 0.02 & 0.06 & & 0.04 & & 0.54 & 13.50 & & 0.02 & 0.61 & 0.06 & $9.3 \mathrm{E}-02$ & $3.2 \mathrm{E}-03$ & 0.004 \\
\hline 4649-HTF-A & 14.35 & 0.1 & 8.59 & & 0.06 & 0.82 & 3.33 & 0.35 & 0.26 & 9.50 & & 0.03 & 0.08 & & 0.05 & & 0.68 & 18.55 & & 0.03 & 0.79 & 0.08 & $1.3 \mathrm{E}-01$ & $8.8 \mathrm{E}-03$ & NA \\
\hline 4728-HTF-B & 10.35 & 0.1 & 5.64 & $<$ & 0.01 & 0.70 & 3.74 & 0.30 & 0.26 & 11.35 & $<$ & 0.01 & 0.07 & & 0.02 & & 0.76 & 13.20 & $<$ & 0.01 & 0.61 & 0.02 & $4.5 \mathrm{E}-03$ & 1.3E-04 & 0.004 \\
\hline 4728-HTF-A & 12.70 & 0.1 & 7.13 & $<$ & 0.01 & 0.80 & 4.70 & 0.34 & 0.31 & 13.75 & $<$ & 0.01 & 0.09 & & 0.02 & & 0.90 & 16.25 & & 0.02 & 0.71 & 0.03 & $6.2 \mathrm{E}-03$ & $2.7 \mathrm{E}-04$ & NA \\
\hline
\end{tabular}

$*$ Note Boron values are 'less than' values; boron was added to SBW simulant at $\sim 0.2 \mathrm{~g} / \mathrm{L}$;

** Note $\mathrm{Hg}<0.004 \mathrm{wt} \%$

$\mathrm{NA}-\mathrm{Hg}$ analysis not performed on after-ashed samples. 
WSRC-STI-2007-00319, REV. 0

Table 4-2. Oxide Composition - Wt\%

\begin{tabular}{|c|c|c|c|c|c|c|c|c|c|c|c|c|c|c|c|c|c|c|c|c|c|c|c|c|}
\hline $\begin{array}{c}\text { Sample } \\
\text { ID }\end{array}$ & $\mathrm{Al} 2 \mathrm{O} 3$ & $\mathrm{~B} 2 \mathrm{O} 3$ & $\mathrm{CaO}$ & & $\mathrm{Cr} 2 \mathrm{O} 3$ & $\mathrm{Fe} 2 \mathrm{O} 3$ & $\mathrm{~K} 2 \mathrm{O}$ & $\mathrm{MgO}$ & $\mathrm{MnO} 2$ & $\mathrm{Na} 2 \mathrm{O}$ & & $\mathrm{NiO}$ & $\mathrm{P2O5}$ & & $\mathrm{PbO}$ & & $\mathrm{SO} 3$ & $\mathrm{SiO} 2$ & & Sro & TiO2 & $\mathrm{ZnO}$ & $\mathrm{Cs} 2 \mathrm{O}$ & $\mathrm{ReO} 2$ \\
\hline $\begin{array}{l}4504- \\
\text { DMR-B } \\
\end{array}$ & 66.80 & 0.32 & 2.55 & & 0.06 & 4.90 & 1.26 & 0.26 & 0.22 & 5.54 & & 0.03 & 0.15 & & 0.01 & $<$ & 0.08 & 10.98 & & 0.02 & 0.41 & 0.11 & $6.5 \mathrm{E}-03$ & $1.4 \mathrm{E}-03$ \\
\hline $\begin{array}{c}4504- \\
\text { DMR-A }\end{array}$ & 63.68 & 0.32 & 3.43 & & 0.07 & 4.16 & 1.70 & 0.30 & 0.27 & 6.96 & & \begin{tabular}{|l|}
0.03 \\
\end{tabular} & 0.19 & & $\begin{array}{l} \\
0.01 \\
\end{array}$ & & 0.10 & 15.21 & $<$ & 0.01 & 0.52 & 0.14 & $6.5 \mathrm{E}-03$ & $1.7 \mathrm{E}-03$ \\
\hline $\begin{array}{c}4531- \\
\text { DMR-B }\end{array}$ & 61.03 & 0.32 & 3.78 & & 0.07 & 5.81 & 1.86 & 0.33 & 0.30 & 6.87 & & 0.03 & 0.14 & & 0.01 & $<$ & 0.08 & 15.99 & & 0.02 & 0.63 & 0.18 & $7.5 \mathrm{E}-03$ & $1.9 \mathrm{E}-03$ \\
\hline $\begin{array}{c}4531- \\
\text { DMR-A } \\
\end{array}$ & 54.51 & 0.32 & 4.55 & & 0.08 & 5.03 & 2.63 & 0.36 & 0.34 & 8.23 & & 0.03 & 0.19 & & \begin{tabular}{|l} 
\\
\end{tabular} & & 0.09 & 20.71 & $<$ & 0.01 & 0.72 & 0.20 & $6.6 \mathrm{E}-03$ & $2.0 \mathrm{E}-03$ \\
\hline $\begin{array}{c}4637- \\
\text { DMR-B }\end{array}$ & 42.89 & 0.32 & 4.78 & & 0.09 & 4.19 & 3.05 & 0.41 & 0.36 & 11.34 & & \begin{tabular}{|l|} 
\\
\end{tabular} & 0.12 & & 0.02 & $<$ & 0.08 & 29.31 & & 0.03 & 1.08 & 0.24 & $1.1 \mathrm{E}-02$ & $3.6 \mathrm{E}-03$ \\
\hline $\begin{array}{l}4637- \\
\text { DMR-A } \\
\end{array}$ & 45.92 & 0.32 & 4.54 & & 0.09 & 4.50 & 2.96 & 0.38 & 0.33 & 11.20 & & 0.06 & 0.10 & & \begin{tabular}{|l} 
\\
\end{tabular} & $<$ & 0.08 & 28.67 & & 0.01 & 0.96 & 0.24 & $1.2 \mathrm{E}-02$ & $1.8 \mathrm{E}-03$ \\
\hline $\begin{array}{c}4726- \\
\text { DMR-B }\end{array}$ & 36.56 & 0.32 & 5.95 & & 0.07 & 4.57 & 4.06 & 0.59 & 0.52 & 17.05 & & 0.03 & 0.18 & $<$ & \begin{tabular}{|l} 
\\
\end{tabular} & & 0.09 & 31.77 & & 0.02 & 1.11 & 0.07 & $4.3 \mathrm{E}-03$ & $9.4 \mathrm{E}-04$ \\
\hline $\begin{array}{c}4726- \\
\text { DMR-A }\end{array}$ & 36.37 & 0.32 & 6.68 & & 0.08 & 3.37 & 4.05 & 0.66 & 0.55 & 17.39 & & 0.03 & 0.19 & $<$ & \begin{tabular}{|l} 
\\
\end{tabular} & & 0.09 & 32.09 & & 0.02 & 1.19 & 0.08 & $2.8 \mathrm{E}-03$ & $1.1 \mathrm{E}-03$ \\
\hline $\begin{array}{l}4508- \\
\text { HTF-B }\end{array}$ & 25.23 & 0.32 & 7.23 & & 0.08 & 1.92 & 3.60 & 0.48 & 0.37 & 13.29 & & 0.04 & 0.18 & & 0.03 & & 1.70 & 29.20 & $<$ & 0.01 & 1.09 & 0.13 & $9.8 \mathrm{E}-02$ & $8.0 \mathrm{E}-03$ \\
\hline $\begin{array}{l}4508- \\
\text { HTF-A }\end{array}$ & 28.34 & 0.32 & 8.85 & & 0.10 & 2.37 & 4.60 & 0.54 & 0.44 & 15.97 & & 0.05 & 0.25 & & \begin{tabular}{|l|}
0.04 \\
\end{tabular} & & 1.75 & 34.33 & & 0.02 & 1.29 & 0.13 & $1.1 \mathrm{E}-01$ & $1.7 \mathrm{E}-02$ \\
\hline $\begin{array}{l}4546- \\
\text { HTF-B }\end{array}$ & 21.92 & 0.32 & 7.15 & & 0.08 & 1.63 & 3.63 & 0.43 & 0.35 & 11.82 & & 0.03 & 0.18 & & \begin{tabular}{|l|} 
\\
\end{tabular} & & 1.34 & 30.59 & & 0.01 & 1.12 & 0.09 & $9.7 \mathrm{E}-02$ & $7.3 \mathrm{E}-03$ \\
\hline $\begin{array}{l}4546- \\
\text { HTF-A }\end{array}$ & 28.15 & 0.32 & 8.58 & & 0.10 & 2.14 & 4.10 & 0.53 & 0.44 & 14.69 & & 0.04 & 0.23 & & 0.05 & & 1.70 & 38.72 & & 0.02 & 1.39 & 0.13 & $1.1 \mathrm{E}-01$ & 2.1E-02 \\
\hline $\begin{array}{l}4649- \\
\text { HTF-B } \\
\end{array}$ & 19.46 & 0.32 & 8.90 & & 0.06 & 0.87 & 2.90 & 0.44 & 0.32 & 9.42 & & \begin{tabular}{|l}
0.02 \\
\end{tabular} & 0.14 & & 0.04 & & 1.34 & 28.88 & & 0.03 & 1.02 & 0.07 & $9.9 \mathrm{E}-02$ & $3.8 \mathrm{E}-03$ \\
\hline $\begin{array}{l}4649- \\
\text { HTF-A }\end{array}$ & 27.12 & 0.32 & 12.01 & & 0.08 & 1.17 & 4.01 & 0.57 & 0.41 & 12.81 & & \begin{tabular}{|l|} 
\\
\end{tabular} & 0.18 & & \begin{tabular}{|l|} 
\\
\end{tabular} & & 1.70 & 39.68 & & 0.04 & 1.31 & 0.10 & $1.4 \mathrm{E}-01$ & $1.0 \mathrm{E}-02$ \\
\hline $\begin{array}{l}4728- \\
\text { HTF-B }\end{array}$ & 19.56 & 0.32 & 7.88 & $<$ & 0.01 & 1.00 & 4.50 & 0.49 & 0.42 & 15.30 & $<$ & 0.01 & 0.16 & & 0.03 & & 1.90 & 28.24 & $<$ & 0.01 & 1.02 & 0.03 & $4.8 \mathrm{E}-03$ & 1.5E-04 \\
\hline $\begin{array}{l}4728- \\
\text { HTF-A }\end{array}$ & 24.00 & 0.32 & 9.97 & $<$ & 0.01 & 1.15 & 5.66 & 0.57 & 0.49 & 18.53 & $\leq$ & 0.01 & 0.20 & & \begin{tabular}{|l|} 
\\
\end{tabular} & & 2.25 & 34.76 & & 0.02 & 1.18 & 0.04 & $6.6 \mathrm{E}-03$ & 3.1E-04 \\
\hline
\end{tabular}

* Note $\mathrm{B}_{2} \mathrm{O}_{3}$ values are 'less than' values; boron was added to SBW simulant at $\sim 0.2 \mathrm{~g} / \mathrm{L}$ 
WSRC-STI-2007-00319, REV. 0

Table 4-2. Oxide Composition - Wt\% - continued

\begin{tabular}{|c|c|c|c|c|c|c|c|c|c|c|c|c|c|}
\hline Sample ID & & $\mathrm{HgO}^{*}$ & & $\mathbf{F}$ & & $\mathrm{NaCl}$ & & $\mathrm{NO} 2$ & & $\mathrm{NO3}$ & Carbon & $\begin{array}{l}\text { Sum } \\
\text { wt\% }\end{array}$ & $\begin{array}{c}\text { Redox } \\
\text { Fe2 }{ }^{+} / \text {Fe total } \\
\end{array}$ \\
\hline 4504-DMR-B & $<$ & 0.005 & $<$ & 0.1 & $<$ & 0.16 & & 1 & $<$ & 0.1 & 0.98 & 96.33 & 0.41 \\
\hline 4504-DMR-A & $<$ & NA & $<$ & 0.1 & $<$ & 0.16 & $<$ & 0.1 & $<$ & 0.1 & 0 & 97.79 & NA \\
\hline 4531-DMR-B & $<$ & 0.005 & $<$ & 0.1 & $<$ & 0.16 & $<$ & 0.1 & $<$ & 0.1 & 0.31 & 98.50 & 0.27 \\
\hline 4531-DMR-A & $<$ & NA & $<$ & 0.1 & $<$ & 0.16 & & 1.16 & $<$ & 0.1 & 0 & 99.83 & NA \\
\hline 4637-DMR-B & $<$ & 0.005 & $<$ & 0.1 & $<$ & 0.16 & & 0.978 & $<$ & 0.1 & 0.8 & 100.76 & 0.44 \\
\hline 4637-DMR-A & $<$ & NA & $<$ & 0.1 & $<$ & 0.16 & & 1.12 & $<$ & 0.1 & 0 & 102.12 & NA \\
\hline 4726-DMR-B & $<$ & 0.005 & $<$ & 0.1 & $<$ & 0.16 & $<$ & 0.1 & $<$ & 0.1 & 0.4 & 104.12 & 0.29 \\
\hline 4726-DMR-A & $<$ & NA & $<$ & 0.1 & $<$ & 0.16 & & 1.15 & $<$ & 0.1 & 0 & 104.98 & NA \\
\hline 4508-HTF-B & $<$ & 0.005 & $<$ & 0.1 & & 0.59 & & 1.99 & $<$ & 0.1 & 13.15 & 101.21 & 1.00 ** \\
\hline 4508-HTF-A & $<$ & NA & $<$ & 0.1 & & 0.81 & & 1.09 & $<$ & 0.1 & 0 & 101.90 & NA \\
\hline 4546-HTF-B & $<$ & 0.005 & $<$ & 0.1 & & 0.47 & & 1.62 & $<$ & 0.1 & 20.87 & 104.25 & $1.00 * \star$ \\
\hline 4546-HTF-A & $<$ & NA & $<$ & 0.1 & & 0.90 & & 1.07 & $<$ & 0.1 & 0 & 103.93 & NA \\
\hline 4649-HTF-B & $<$ & 0.005 & $<$ & 0.1 & & 0.45 & & 1.91 & $<$ & 0.1 & 26.47 & 103.65 & $1.00^{* *}$ \\
\hline 4649-HTF-A & $<$ & NA & $<$ & 0.1 & & 0.89 & & 1.27 & $<$ & 0.1 & 0 & 104.42 & NA \\
\hline 4728-HTF-B & $<$ & 0.005 & $<$ & 0.1 & & 0.91 & & 2.52 & $<$ & 0.1 & 19.49 & 104.29 & $1.00^{* *}$ \\
\hline 4728-HTF-A & $<$ & NA & $<$ & 0.1 & & 1.43 & & 1.25 & $<$ & 0.1 & 0 & 102.37 & NA \\
\hline
\end{tabular}

* Note NA - Hg analysis not performed on after-ashed samples.

** Essentially all iron in the reduced +2 state for the HTF samples. 


\subsection{CRYSTALLINE PHASES}

In Table 4-3 the various crystalline phases observed in the coarse-sieved DMR and unsieved HTF samples are summarized. The individual XRD spectrums for each powder sample are collected in an Appendix A to this report. The DMR 4504 sample appears to have major crystalline species corundum attributed to the alumina starting bed and alumina added during processing for particle size control. The DMR 4531 sample appears to also show major corundum, some nepheline and minor phase of quartz $\left(\mathrm{SiO}_{2}\right)$. The last two DMR samples appear to be mostly nepheline and sodium aluminum silicate, with only minor phases of corundum and quartz. These XRD spectra and crystalline identification are in agreement with the progression of DMR samples shown previously in the ternary diagram, i.e., progressive 'turnover' of the starting alumina (corundum phase) bed in going from DMR-4504 through final DMR-4726. All HTF samples are similar with major nepheline and sodium aluminum silicate phases with minor conundrum and quartz phases present. Solid mineral phases produced from using clay as coreactant in the FBSR are sodium aluminosilicates (NAS) comprised of nepheline group crystals and sodalite group crystals including nosean [Pareizs et al., 2005]. These DMR and HTF samples appear to have mainly the nepheline and sodium aluminum silicate crystals. If the sodalite/nosean crystals are present, they are likely below the nominal $\sim 1-2$ vol\% crystalline content of the powder-XRD method used in this work.

Table 4-3. XRD Crystalline Phases

\begin{tabular}{|c|c|c|c|c|c|}
\hline $\begin{array}{l}\text { DMR } \\
4504\end{array}$ & $\begin{array}{l}\text { (Major) } \\
\text { Nepheline - } \\
\mathrm{NaAlSiO}_{4} \\
\end{array}$ & $\begin{array}{l}\text { (Major) } \\
\text { Diaoyudaoite - } \\
\mathrm{NaAl}_{11} \mathrm{O}_{17} \\
\end{array}$ & $\begin{array}{l}\text { (Major) } \\
\text { Corundum - } \\
\mathrm{Al}_{2} \mathrm{O}_{3} \\
\end{array}$ & - & - \\
\hline $\begin{array}{l}\text { DMR } \\
4531\end{array}$ & $\begin{array}{l}\text { (Major) } \\
\text { Nepheline - } \\
\mathrm{NaAlSiO}_{4}\end{array}$ & $\begin{array}{l}\text { (Major) } \\
\text { Diaoyudaoite - } \\
\mathrm{NaAl}_{11} \mathrm{O}_{17}\end{array}$ & $\begin{array}{l}\text { (Major) } \\
\text { Corundum - } \\
\mathrm{Al}_{2} \mathrm{O}_{3}\end{array}$ & $\begin{array}{l}\text { (Minor) Quartz } \\
-\mathrm{SiO}_{2}\end{array}$ & - \\
\hline $\begin{array}{l}\text { DMR } \\
4637\end{array}$ & $\begin{array}{l}\text { (Major) } \\
\text { Nepheline - } \\
\mathrm{NaAlSiO}_{4}\end{array}$ & - & $\begin{array}{l}\text { (Minor) } \\
\text { Corundum - } \\
\mathrm{Al}_{2} \mathrm{O}_{3}\end{array}$ & Quartz $-\mathrm{SiO}_{2}$ & $\begin{array}{l}\text { (Major) Sodium } \\
\text { Aluminum Silicate - } \\
\mathrm{Na}_{1.75} \mathrm{Al}_{1.75} \mathrm{Si}_{0.25} \mathrm{O}_{4}\end{array}$ \\
\hline $\begin{array}{l}\text { DMR } \\
4726\end{array}$ & $\begin{array}{l}\text { (Major) } \\
\text { Nepheline - } \\
\mathrm{NaAlSiO}_{4} \\
\end{array}$ & - & $\begin{array}{l}\text { (Minor) } \\
\text { Corundum - } \\
\mathrm{Al}_{2} \mathrm{O}_{3} \\
\end{array}$ & $\begin{array}{l}\text { (Minor) Quartz } \\
-\mathrm{SiO}_{2}\end{array}$ & $\begin{array}{l}\text { (Major) Sodium } \\
\text { Aluminum Silicate - } \\
\mathrm{Na}_{1.75} \mathrm{Al}_{1.75} \mathrm{Si}_{0.25} \mathrm{O}_{4}\end{array}$ \\
\hline $\begin{array}{l}\text { HTF } \\
4508\end{array}$ & $\begin{array}{l}\text { (Major) } \\
\text { Nepheline - } \\
\mathrm{NaAlSiO}_{4}\end{array}$ & - & $\begin{array}{l}\text { (Minor) } \\
\text { Corundum - } \\
\mathrm{Al}_{2} \mathrm{O}_{3}\end{array}$ & $\begin{array}{l}\text { (Minor) Quartz } \\
-\mathrm{SiO}_{2}\end{array}$ & $\begin{array}{l}\text { (Major) Sodium } \\
\text { Aluminum Silicate - } \\
\mathrm{Na}_{1.75} \mathrm{Al}_{1.75} \mathrm{Si}_{0.25} \mathrm{O}_{4}\end{array}$ \\
\hline $\begin{array}{l}\text { HTF } \\
4546\end{array}$ & $\begin{array}{l}\text { (Major) } \\
\text { Nepheline - } \\
\mathrm{NaAlSiO}_{4} \\
\end{array}$ & - & $\begin{array}{l}\text { (Minor) } \\
\text { Corundum - } \\
\mathrm{Al}_{2} \mathrm{O}_{3} \\
\end{array}$ & $\begin{array}{l}\text { (Minor) Quartz } \\
-\mathrm{SiO}_{2}\end{array}$ & $\begin{array}{l}\text { (Major) Sodium } \\
\text { Aluminum Silicate - } \\
\mathrm{Na}_{1.75} \mathrm{Al}_{1.75} \mathrm{Si}_{0.25} \mathrm{O}_{4}\end{array}$ \\
\hline $\begin{array}{l}\mathrm{HTF} \\
4649\end{array}$ & $\begin{array}{l}\text { (Major) } \\
\text { Nepheline - } \\
\mathrm{NaAlSiO}_{4} \\
\end{array}$ & - & $\begin{array}{l}\text { (Minor) } \\
\text { Corundum - } \\
\mathrm{Al}_{2} \mathrm{O}_{3}\end{array}$ & $\begin{array}{l}\text { (Minor) Quartz } \\
-\mathrm{SiO}_{2}\end{array}$ & $\begin{array}{l}\text { (Major) Sodium } \\
\text { Aluminum Silicate - } \\
\mathrm{Na}_{1.75} \mathrm{Al}_{1.75} \mathrm{Si}_{0.25} \mathrm{O}_{4}\end{array}$ \\
\hline $\begin{array}{l}\mathrm{HTF} \\
4728\end{array}$ & $\begin{array}{l}\text { (Major) } \\
\text { Nepheline - } \\
\mathrm{NaAlSiO}_{4} \\
\end{array}$ & - & $\begin{array}{l}\text { (Minor) } \\
\text { Corundum - } \\
\mathrm{Al}_{2} \mathrm{O}_{3} \\
\end{array}$ & $\begin{array}{l}\text { (Minor) Quartz } \\
-\mathrm{SiO}_{2}\end{array}$ & $\begin{array}{l}\text { (Major) Sodium } \\
\text { Aluminum Silicate- } \\
\mathrm{Na}_{1.75} \mathrm{Al}_{1.75} \mathrm{Si}_{0.25} \mathrm{O}_{4}\end{array}$ \\
\hline
\end{tabular}




\subsection{DURABILITY TESTING}

Table 4-4 shows the results of surface area to volume ratios calculated from either measured BET surface area or from particle size measurements and density. The HTF samples that were sieved through 200 mesh sieves all showed average particle diameters in the range of 7 - 13 microns. Similar measurements on the DMR samples sieved through 100-200 mesh sieves measured average particle diameters similar to those of glass put through similar sieves, in the range of $106-115$ microns. The BET surface areas are all greater than corresponding geometric surface areas. The ratio of the BET surface area to geometric surface area is calculated as the 'surface roughness' shown in the last column of Table 4-4. The BET surface areas for the HTF samples are 15 to 36 times larger than the geometric surface areas. The BET surface areas for the DMR samples are 135 to 246 times larger than the geometric surface areas. 
WSRC-STI-2007-00319, REV. 0

Table 4-4. BET and Geometric Surface Area Data

\begin{tabular}{|c|c|c|c|c|c|c|c|c|c|}
\hline & $\begin{array}{c}\text { Sample } \\
\text { Mass } \\
(\mathrm{g})\end{array}$ & $\begin{array}{c}\begin{array}{c}\text { Leachant } \\
\text { Volume } \\
(\mathrm{mL})\end{array} \\
\end{array}$ & $\begin{array}{c}\text { BET } \\
\text { surface } \\
\text { area } \\
\left(\mathrm{m}^{2} / \mathrm{g}\right) \\
\end{array}$ & $\begin{array}{l}\text { SA/V } \\
\left(\mathbf{m}^{-1}\right)\end{array}$ & $\begin{array}{c}\text { Particle } \\
\text { Diameter } \\
\text { microns }\end{array}$ & $\begin{array}{c}\text { Particle } \\
\text { Density } \\
\text { g/mL }\end{array}$ & $\begin{array}{c}\text { Geometric } \\
\text { surface area } \\
\left(\mathrm{m}^{2} / \mathrm{g}\right)\end{array}$ & $\begin{array}{l}\text { SA/V } \\
\left(\mathbf{m}^{-1}\right)\end{array}$ & $\begin{array}{c}\text { Surface } \\
\text { Roughness }_{\mathrm{SA}_{\mathrm{BET}} / \mathrm{SA}_{\mathrm{geo}}}\end{array}$ \\
\hline HTF4508-1 P76 & 1.508 & 15.03 & 4.526 & 454116 & 7.182 & 2.794 & 0.2990 & 29997 & 15 \\
\hline HTF4508-2 P81 & 1.498 & 15.001 & 4.526 & 451976 & 7.182 & 2.794 & 0.2990 & 29855 & 15 \\
\hline HTF4508-3 P83 & 1.504 & 15.072 & 4.526 & 451649 & 7.182 & 2.794 & 0.2990 & 29834 & 15 \\
\hline Avg & & & & 452580 & & & & 29895 & \\
\hline Stdev & & & & 1340 & & & & 88 & \\
\hline HTF4546-1 P86 & 1.5 & 15.018 & 5.625 & 561836 & 13.87 & 2.748 & 0.1574 & 15723 & 36 \\
\hline HTF4546-2 P92 & 1.5 & 14.995 & 5.625 & 562698 & 13.87 & 2.748 & 0.1574 & 15747 & 36 \\
\hline HTF4546-3 P93 & 1.498 & 15.05 & 5.625 & 559894 & 13.87 & 2.748 & 0.1574 & 15669 & 36 \\
\hline Avg & & & & 561476 & & & & 15713 & \\
\hline Stdev & & & & 1436 & & & & 40 & \\
\hline HTF4649-1 P97 & 1.507 & 15.091 & 6.413 & 640378 & 11.74 & 2.599 & 0.1966 & 19635 & 33 \\
\hline HTF4649-2 P98 & 1.504 & 15.004 & 6.413 & 642809 & 11.74 & 2.599 & 0.1966 & 19710 & 33 \\
\hline HTF4649-3 P99 & 1.499 & 15.008 & 6.413 & 640501 & 11.74 & 2.599 & 0.1966 & 19639 & 33 \\
\hline Avg & & & & 641229 & & & & 19661 & \\
\hline Stdev & & & & 1369 & & & & 42 & \\
\hline HTF4728-1 P100 & 1.501 & 15.084 & 5.1939 & 516842 & 12.2 & 2.646 & 0.1858 & 18493 & 28 \\
\hline HTF4728-2 P100 & 1.604 & 15.038 & 5.1939 & 553998 & 12.2 & 2.646 & 0.1858 & 19822 & 28 \\
\hline HTF4728-3 P100 & 1.501 & 15.003 & 5.1939 & 519632 & 12.2 & 2.646 & 0.1858 & 18593 & 28 \\
\hline Avg & & & & 530157 & & & & 18969 & \\
\hline Stdev & & & & 20693 & & & & 740 & \\
\hline DMR4726-1 P103 & 1.5 & 15.029 & 2.82 & 281456 & 104.9 & 2.736 & 0.0209 & 2087 & 135 \\
\hline DMR4726-2 P104 & 1.503 & 15.026 & 2.82 & 282075 & 104.9 & 2.736 & 0.0209 & 2091 & 135 \\
\hline DMR4726-3 P110 & 1.505 & 15.013 & 2.82 & 282695 & 104.9 & 2.736 & 0.0209 & 2096 & 135 \\
\hline Avg & & & & 282075 & & & & 2091 & \\
\hline Stdev & & & & 620 & & & & 5 & \\
\hline DMR4637-1 P112 & 1.504 & 15.06 & 4.279 & 427362 & 115.3 & 2.986 & 0.0174 & 1740 & 246 \\
\hline DMR4637-2 P113 & 1.503 & 15.12 & 4.279 & 425383 & 115.3 & 2.986 & 0.0174 & 1732 & 246 \\
\hline DMR4637-3 P114 & 1.502 & 15.042 & 4.279 & 427304 & 115.3 & 2.986 & 0.0174 & 1740 & 246 \\
\hline Avg & & & & 426683 & & & & 1737 & \\
\hline Stdev & & & & 1126 & & & & 5 & \\
\hline DMR4531-1 P121 & 1.503 & 15.032 & 3.492 & 349184 & 112.4 & 3.155 & 0.0169 & 1692 & 206 \\
\hline DMR4531-2 P122 & 1.5 & 15.044 & 3.492 & 348209 & 112.4 & 3.155 & 0.0169 & 1687 & 206 \\
\hline DMR4531-3 P170 & 1.5 & 15.046 & 3.492 & 348162 & 112.4 & 3.155 & 0.0169 & 1687 & 206 \\
\hline Avg & & & & 348518 & & & & 1688 & \\
\hline Stdev & & & & 577 & & & & 3 & \\
\hline DMR4504-1 P171 & 1.501 & 15.036 & 2.515 & 251095 & 106.5 & 3.204 & 0.0176 & 1755 & 143 \\
\hline DMR4504-2 P172 & 1.503 & 15.029 & 2.515 & 251547 & 106.5 & 3.204 & 0.0176 & 1759 & 143 \\
\hline DMR4504-3 P178 & 1.498 & 15.012 & 2.515 & 250994 & 106.5 & 3.204 & 0.0176 & 1755 & 143 \\
\hline Avg & & & & 251212 & & & & 1756 & \\
\hline Stdev & & & & 294 & & & & 2 & \\
\hline
\end{tabular}


All DMR and HTF samples were durability tested in triplicate per the PCT procedure. Glass standards were used to ensure test control as required by the PCT procedure. The standard glasses agreed with previous round robin testing of the glasses for the ARM-1 glass [Jantzen et al., 1995], the EA glass [Jantzen et al., 1993], and the LRM glass [Ebert and Wolf, 2000]. Table 4-5 shows $\mathrm{pH}$ and concentration results for the standard glasses and Table 4-6 shows the results on a normalized concentration basis.

Table 4-5. PCT Leachate Standards and Blanks (ppm)

\begin{tabular}{|c|c|c|c|c|c|c|c|c|c|c|}
\hline Sample ID & pH & & Al & & B & & $\mathbf{L i}$ & & $\mathrm{Na}$ & Si \\
\hline Blank 1 & 7.05 & $<$ & 0.11 & $<$ & 0.11 & $<$ & 1.10 & $<$ & 0.11 & 0.16 \\
\hline Blank 2 & 6.28 & $<$ & 0.11 & $<$ & 0.11 & $<$ & 1.10 & $<$ & 0.11 & 0.18 \\
\hline ARM 1 -P64 & 10.38 & & 4.40 & & 18.9 & & 15.9 & & 42.3 & 68.4 \\
\hline ARM 2 - P65 & 10.35 & & 4.20 & & 18.9 & & 16.0 & & 42.2 & 69.9 \\
\hline ARM 3 - P67 & 10.36 & & 4.15 & & 17.1 & & 15.3 & & 42.4 & 66.5 \\
\hline Average & 10.36 & & 4.25 & & & & & & 42.31 & 68.27 \\
\hline St.Dev. & 0.02 & & 0.13 & & & & & & $\mathbf{0 . 0 7}$ & 1.72 \\
\hline Avg.(St.dev.) * & $10.17(0.29)$ & & $4.85(0.5)$ & & & & & & $36.22(2.45)$ & $61.23(4.07)$ \\
\hline \multicolumn{11}{|c|}{ *See Jantzen et al., 1995} \\
\hline EA-1 P68 & 11.71 & $<$ & 2.0 & & 492.0 & & 161.6 & & 1384.9 & 793.7 \\
\hline EA-2 P69 & 11.77 & $<$ & 2.0 & & 493.6 & & 164.3 & & 1428.5 & 794.5 \\
\hline EA-3 P70 & 11.75 & $<$ & 2.0 & & 493.4 & & 159.2 & & 1364.8 & 787.9 \\
\hline Average & 11.74 & & & & 493.0 & & 161.7 & & 1392.7 & 792.0 \\
\hline St.Dev. & 0.03 & & & & 0.9 & & 2.6 & & 32.6 & 3.6 \\
\hline Avg.(St.dev.) $* *$ & $11.85(0.1)$ & & & & $587(43)$ & & $190(14.5)$ & & $1662(112)$ & $893(86)$ \\
\hline \multicolumn{11}{|c|}{ **See Jantzen et al., 1993} \\
\hline LRM-1 P71 & 10.96 & & 14.1 & & 25.7 & $<$ & 2.8 & & 161.2 & 83.7 \\
\hline LRM-2 P72 & 10.93 & & 13.2 & & 23.4 & $<$ & 2.8 & & 158.7 & 76.2 \\
\hline LRM-3 P74 & 11.05 & & 14.7 & & 22.7 & $<$ & 2.8 & & 152.8 & 106.1 \\
\hline Average & 10.98 & & 13.98 & & 23.93 & & & & 157.58 & 88.65 \\
\hline St.Dev. & 0.06 & & 0.74 & & 1.57 & & & & 4.34 & 15.60 \\
\hline Avg.(St.dev.) $* * *$ & $10.92(0.092)$ & & $14.3(2.61)$ & & $26.7(1.83)$ & & & & $160(11.5)$ & $82(3.53)$ \\
\hline
\end{tabular}


Table 4-6. PCT Leachate Standards and Blanks (g/L)

\begin{tabular}{|c|c|c|c|c|c|}
\hline Sample ID & Al & $\mathbf{B}$ & $\mathbf{L i}$ & $\mathrm{Na}$ & Si \\
\hline ARM 1 -P64 & 0.149 & NA & $\mathrm{NA}$ & 0.590 & 0.315 \\
\hline ARM 2 - P65 & 0.142 & NA & NA & 0.589 & 0.322 \\
\hline ARM 3 - P67 & 0.141 & NA & NA & 0.591 & 0.306 \\
\hline Average & 0.144 & NA & NA & 0.590 & 0.314 \\
\hline St.Dev. & 0.004 & NA & NA & 0.001 & 0.008 \\
\hline Avg.(St.dev.)* & $0.155(0.0172)$ & NA & NA & $0.505(0.0539)$ & $0.282(0.03)$ \\
\hline \multicolumn{6}{|c|}{ *See Jantzen et al., 1995} \\
\hline EA-1 P68 & NA & 14.10 & 8.21 & 11.08 & 3.48 \\
\hline EA-2 P69 & NA & 14.14 & 8.35 & 11.43 & 3.49 \\
\hline EA-3 P70 & NA & 14.14 & 8.09 & 10.92 & 3.46 \\
\hline Average & NA & 14.13 & 8.22 & 11.14 & 3.48 \\
\hline St.Dev. & $\mathrm{NA}$ & 0.02 & 0.13 & 0.26 & 0.02 \\
\hline Avg.(St.dev.)** & NA & $16.695(1.222)$ & $9.565(0.735)$ & $13.346(0.902)$ & $3.922(0.376)$ \\
\hline \multicolumn{6}{|c|}{ **See Jantzen et al., 1993} \\
\hline LRM-1 P71 & 0.28 & 1.05 & NA & 1.08 & 0.330 \\
\hline LRM-2 P72 & 0.26 & 0.96 & NA & 1.07 & 0.301 \\
\hline LRM-3 P74 & 0.29 & 0.93 & NA & 1.03 & 0.419 \\
\hline Average & 0.28 & 0.98 & NA & 1.06 & 0.35 \\
\hline St.Dev. & 0.01 & 0.06 & NA & 0.03 & 0.06 \\
\hline Avg. *** & 0.28 & 1.10 & NA & 1.08 & 0.32 \\
\hline St.Dev. $* * *$ & 0.05 & 0.08 & NA & 0.08 & 0.01 \\
\hline
\end{tabular}

$\mathrm{NA}=$ not applicable; the measured normalized concentrations values not shown - reference values are not available for comparison

Triplicate PCT leachate concentration and $\mathrm{pH}$ data for the DMR and HTF samples are shown in Table 4-7. All $\mathrm{pH}$ values for the DMR and HTF sample leachates were in the range of 12 to 12.7. This $\mathrm{pH}$ range is slightly higher than the reported average from the EA glass of $\sim 11.8$. The leachate concentration data shown in Table 4-7 were normalized using the elemental compositions shown previously in Table 4-1 and the BET surface area shown in Table 4-4, via Equations 1, 2 and 4 . The normalized release data $\left(\mathrm{NL}_{\mathrm{i}}\right)$ are shown in Table 4-8. All of the normalized releases for the DMR and HTF samples were less than $1 \mathrm{~g} / \mathrm{m}^{2}$. Because the elemental concentration for $\mathrm{S}$ in sample DMR-4637 was below detection, the NL(S) values in Table 4-8 are shown as '>' values. 
WSRC-STI-2007-00319, REV. 0

Table 4-7. PCT Leachate Concentrations (ppm)

\begin{tabular}{|c|c|c|c|c|c|c|c|c|}
\hline Sample ID & $\mathbf{p H}$ & Al & $\mathbf{K}$ & $\mathrm{Na}$ & $\mathbf{S}$ & Si & Cs & $\mathbf{R e}$ \\
\hline HTF4508-1 P76 & 12.81 & 1971.3 & 574.7 & 5479.5 & 958.9 & 15.4 & 10.5 & 30.2 \\
\hline HTF4508-2 P81 & 12.65 & 2089.7 & 573.0 & 5426.6 & 1011.2 & 16.2 & 11.0 & 29.6 \\
\hline HTF4508-3 P83 & 12.79 & 1916.5 & 574.9 & 5413.2 & 961.6 & 15.9 & 10.7 & 29.6 \\
\hline Avg. & 12.75 & 1992.5 & 574.2 & 5439.8 & $\mathbf{9 7 7 . 2}$ & 15.8 & 10.7 & 29.8 \\
\hline St. Dev. & 0.09 & 88.6 & 1.1 & 35.0 & 29.4 & 0.4 & 0.3 & 0.4 \\
\hline HTF4546-1 P86 & 12.37 & 2232.0 & 480.1 & 4087.7 & 798.1 & 22.0 & 19.8 & 25.5 \\
\hline HTF4546-2 P92 & 12.38 & 2173.6 & 478.4 & 3916.4 & 797.3 & 19.3 & 20.7 & 25.5 \\
\hline HTF4546-3 P93 & 12.39 & 2090.9 & 456.0 & 3781.4 & 831.4 & 18.9 & 21.4 & 25.4 \\
\hline Avg. & 12.38 & 2165.5 & 471.5 & 3928.5 & 808.9 & 20.1 & 20.6 & 25.5 \\
\hline St. Dev. & 0.01 & 70.9 & 13.5 & 153.5 & 19.4 & 1.7 & 0.8 & 0.0 \\
\hline HTF4649-1 P97 & 12.12 & 945.2 & 266.6 & 2224.1 & 581.0 & 7.6 & 19.8 & 22.0 \\
\hline HTF4649-2 P98 & 12.14 & 926.4 & 264.9 & 2210.6 & 565.8 & 7.9 & 20.0 & 22.1 \\
\hline HTF4649-3 P99 & 12.15 & 929.7 & 258.4 & 2192.3 & 546.7 & 5.9 & 20.3 & 22.3 \\
\hline Avg. & 12.14 & 933.8 & 263.3 & 2209.0 & 564.5 & 7.1 & 20.0 & 22.1 \\
\hline St. Dev. & 0.02 & 10.1 & 4.4 & 15.9 & 17.2 & 1.1 & 0.3 & 0.2 \\
\hline HTF4728-1 P100 & 12.50 & 1586.0 & 895.6 & 5850.5 & 1178.4 & 13.8 & 0.4 & 1.3 \\
\hline HTF4728-2 P100 & 12.88 & 1624.0 & 870.4 & 5784.0 & 1190.2 & 13.3 & 1.6 & 1.4 \\
\hline HTF4728-3 P100 & 12.61 & 1579.5 & 829.8 & 5781.3 & 1286.3 & 12.7 & 0.5 & 1.4 \\
\hline Avg. & 12.66 & 1596.5 & 865.3 & 5805.2 & 1218.3 & 13.2 & 0.8 & 1.4 \\
\hline St. Dev. & 0.20 & 24.0 & 33.2 & 39.2 & 59.2 & 0.6 & 0.7 & 0.0 \\
\hline DMR4726-1 P103 & 12.40 & 2611.9 & 79.7 & 3417.8 & 25.3 & 62.5 & 0.1 & 0.2 \\
\hline DMR4726-2 P104 & 12.58 & 2546.5 & 79.2 & 3376.9 & 29.1 & 63.4 & 0.1 & 0.2 \\
\hline DMR4726-3 P110 & 12.57 & 2595.1 & 78.2 & 3449.1 & 27.5 & 62.3 & 0.1 & 0.2 \\
\hline Avg. & 12.52 & 2584.5 & 79.0 & 3414.6 & 27.3 & 62.7 & 0.1 & 0.2 \\
\hline St. Dev. & 0.10 & 34.0 & 0.8 & 36.2 & 1.9 & 0.6 & 0.0 & 0.0 \\
\hline DMR4637-1 P112 & 12.01 & 649.4 & 20.3 & 930.9 & 9.8 & 43.5 & 1.4 & 0.8 \\
\hline DMR4637-2 P113 & 12.08 & 651.9 & 19.7 & 927.8 & 9.6 & 43.5 & 1.3 & 0.8 \\
\hline DMR4637-3 P114 & 12.09 & 650.2 & 20.1 & 971.1 & 10.7 & 44.9 & 1.3 & 0.8 \\
\hline Avg. & 12.06 & 650.5 & 20.0 & 943.2 & 10.0 & 44.0 & 1.3 & 0.8 \\
\hline St. Dev. & 0.04 & 1.3 & 0.3 & 24.2 & 0.6 & 0.8 & 0.0 & 0.0 \\
\hline DMR4531-1 P121 & 12.08 & 908.5 & 31.8 & 1237.3 & 26.8 & 46.0 & 0.8 & 0.7 \\
\hline DMR4531-2 P122 & 12.06 & 914.8 & 33.2 & 1202.1 & 27.1 & 45.2 & 0.8 & 0.7 \\
\hline DMR4531-3 P170 & 12.15 & 920.3 & 32.5 & 1231.7 & 25.0 & 44.5 & 0.8 & 0.7 \\
\hline Avg. & 12.10 & 914.5 & 32.5 & 1223.7 & 26.3 & 45.2 & 0.8 & 0.7 \\
\hline St. Dev. & 0.05 & 5.9 & 0.7 & 19.0 & 1.1 & 0.7 & 0.0 & 0.0 \\
\hline DMR4504-1 P171 & 12.27 & 1292.7 & 45.2 & 1820.4 & 38.0 & 56.4 & 1.0 & 0.5 \\
\hline DMR4504-2 P172 & 12.32 & 1314.5 & 44.0 & 1913.2 & 34.5 & 52.6 & 0.9 & 0.6 \\
\hline DMR4504-3 P178 & 12.25 & 1311.3 & 44.0 & 1823.6 & 33.7 & 52.1 & 0.9 & 0.5 \\
\hline Avg. & 12.28 & 1306.2 & 44.4 & 1852.4 & 35.4 & 53.7 & 1.0 & 0.5 \\
\hline St. Dev. & 0.04 & 11.8 & 0.7 & 52.7 & 2.3 & 2.4 & 0.1 & 0.0 \\
\hline
\end{tabular}


WSRC-STI-2007-00319, REV. 0

Table 4-8. Normalized PCT Leachate Release $\left(\mathrm{g} / \mathrm{m}^{2}\right)$ Using BET Surface Area

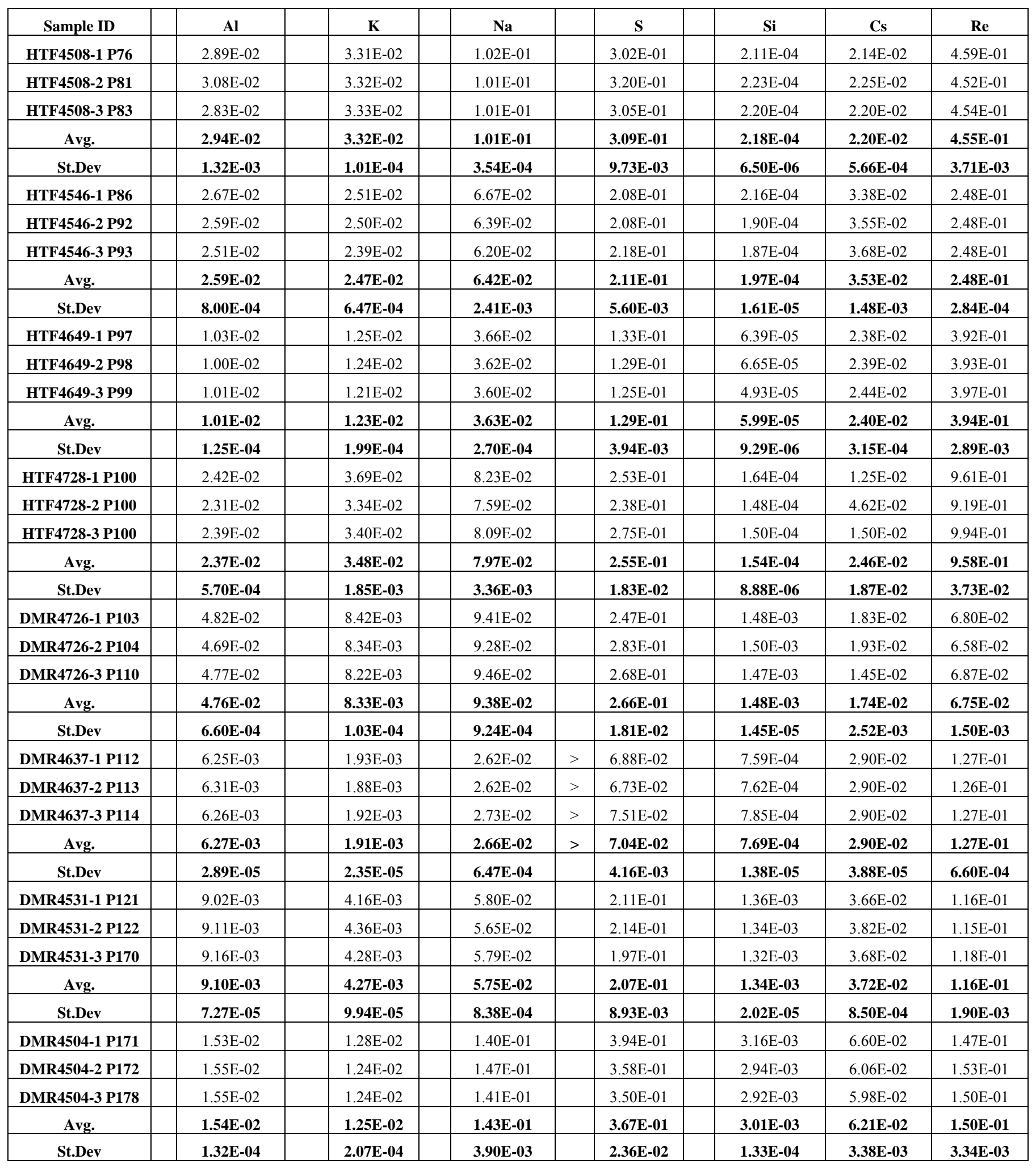


WSRC-STI-2007-00319, REV. 0

\subsubsection{Comparison of Normalized Release to High-Level Waste}

Normalized sodium and silicon release values for the DMR and HTF samples are shown in comparison to those of the High Level Waste (HLW) reference EA glass in Figure 4-1 and Figure 4-2. The EA glass normalized release values are calculated from the data shown previously in Table 4-6 and using Equations 1, 2 and 3. Normalized release EA data are shown as the average and as the average minus two standard deviations. Comparison of a given HLW waste form normalized release vs. the EA values minus two standard deviations is one method suggested by the Waste Acceptance Product Specifications (WAPS) for showing adequate waste form durability [WAPS 2003]. Figure 4-1 and Figure 4-2 clearly show that the normalized releases for $\mathrm{Na}$ and $\mathrm{Si}$ from the FBSR products are orders of magnitude lower than the corresponding average EA normalized release values.

\subsubsection{Comparison of Normalized Release to Low Activity Waste}

Normalized releases for $\mathrm{Na}$ and $\mathrm{Si}$ and from other elements in the DMR and HTF samples are also compared to the normalized release rate from the Low Activity Waste (LAW) target LRM glass (plotted on both a BET surface area and a geometric surface area basis) in Figure 4-3 and Figure 4-4. The Hanford River Protection Project (RPP) contract specification [WTP Contract 2007] of $2 \mathrm{~g} / \mathrm{m}^{2}$ is also plotted in the figure. Figure 4-3 and Figure 4-4 show that the normalized release for the major FBSR product elements (Al, Na, and $\mathrm{Si}$ ), as well as the minor elements (K, S, Cs and $\mathrm{Re}$ ) are all less than half of the $2 \mathrm{~g} / \mathrm{m}^{2}$ limit. Careful comparison of the various normalized release elements (Al, Na, Si, K, S, Cs and Re) for the DMR and HTF samples indicates that NL(S) and NL(Re) appear to be highest. The NL(Re) values for the HTF samples shown in Figure 4-4 also appear higher than the corresponding NL(Re) values for the DMR samples. The oxide forms of both $\mathrm{S}$ and Re are known to be REDOX-dependent. For instance sulfur can exist in an oxidized state as $\mathrm{Na}_{2} \mathrm{SO}_{4}$ or in a reduced state of $\mathrm{Na}_{2} \mathrm{~S}$. Similarly, $\mathrm{Re}$ can be in the +7 state as $\mathrm{Re}_{2} \mathrm{O}_{7}$ or $\mathrm{NaReO}_{4}$ or in a more reduced state of $\mathrm{Re}_{2} \mathrm{O}$. A possible explanation for the higher $\mathrm{NL}(\mathrm{Re})$ values for the HTF samples (very reduced REDOX values from Table 4-2) vs. the NL(Re) values for the DMR samples (more oxidized REDOX values from Table 4-2) is that the more oxidized form of $\mathrm{NaReO}_{4}$ can be incorporated into the cage-like structure of the product minerals but $\mathrm{ReO}_{2}$ cannot. The durability of these $\mathrm{S} / \mathrm{Re}$ oxide forms and their incorporation into various mineral phases has been previously considered and discussed by Lorier et al., 2005, in durability testing that used single-pass flowthrough (SPFT) testing. 


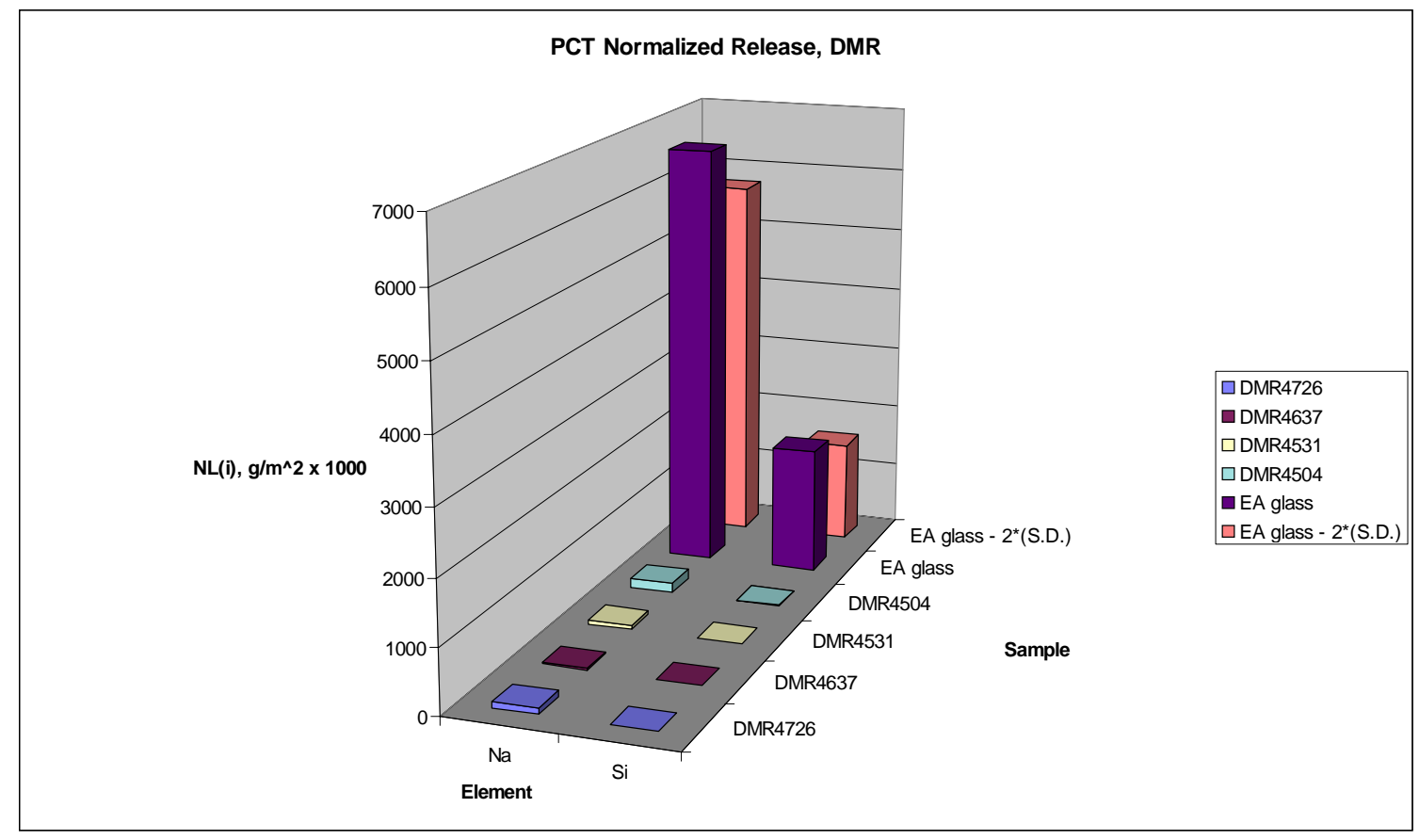

Figure 4-1. Normalized Release for DMR vs. EA Glass

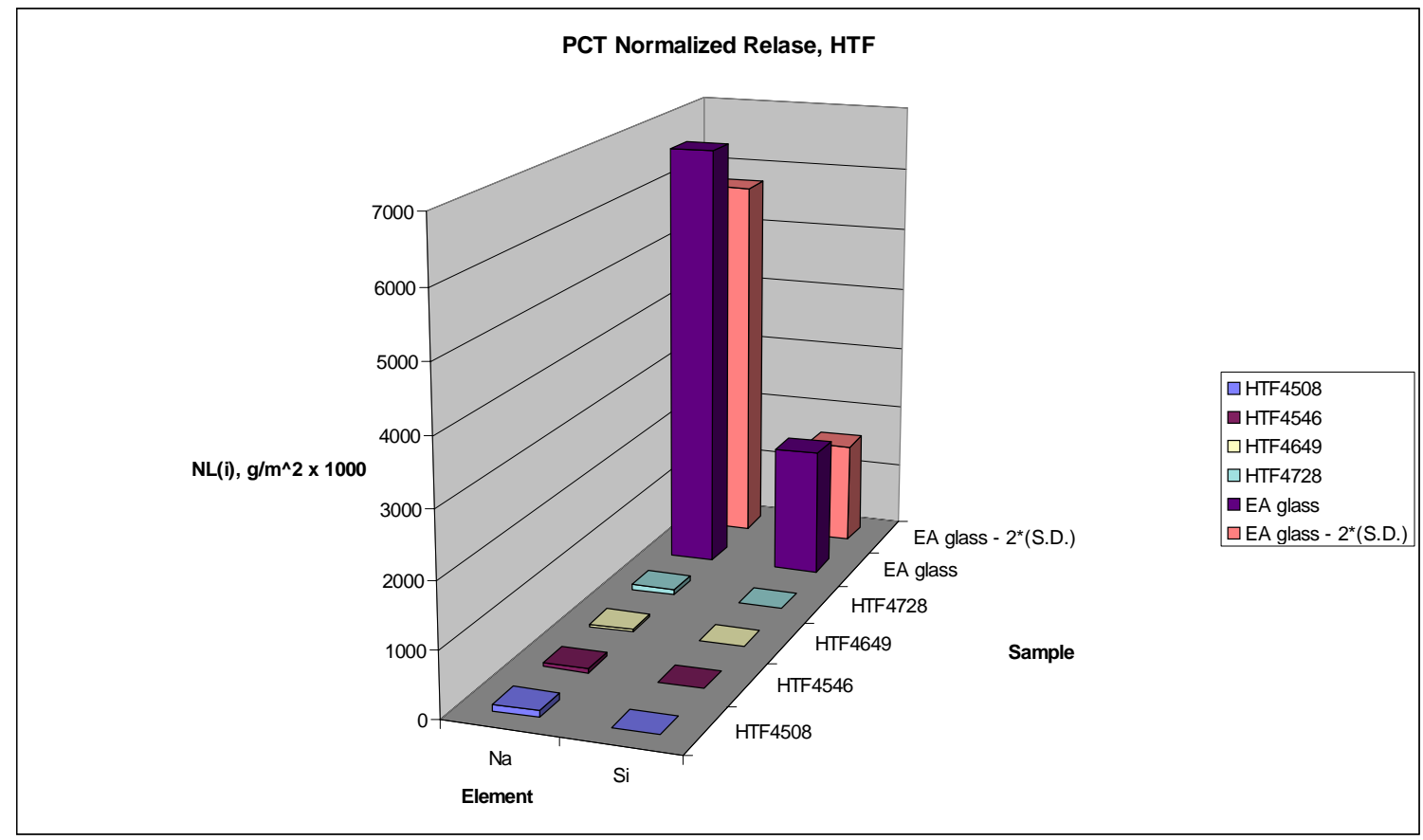

Figure 4-2. Normalized Release for HTF vs. EA Glass 


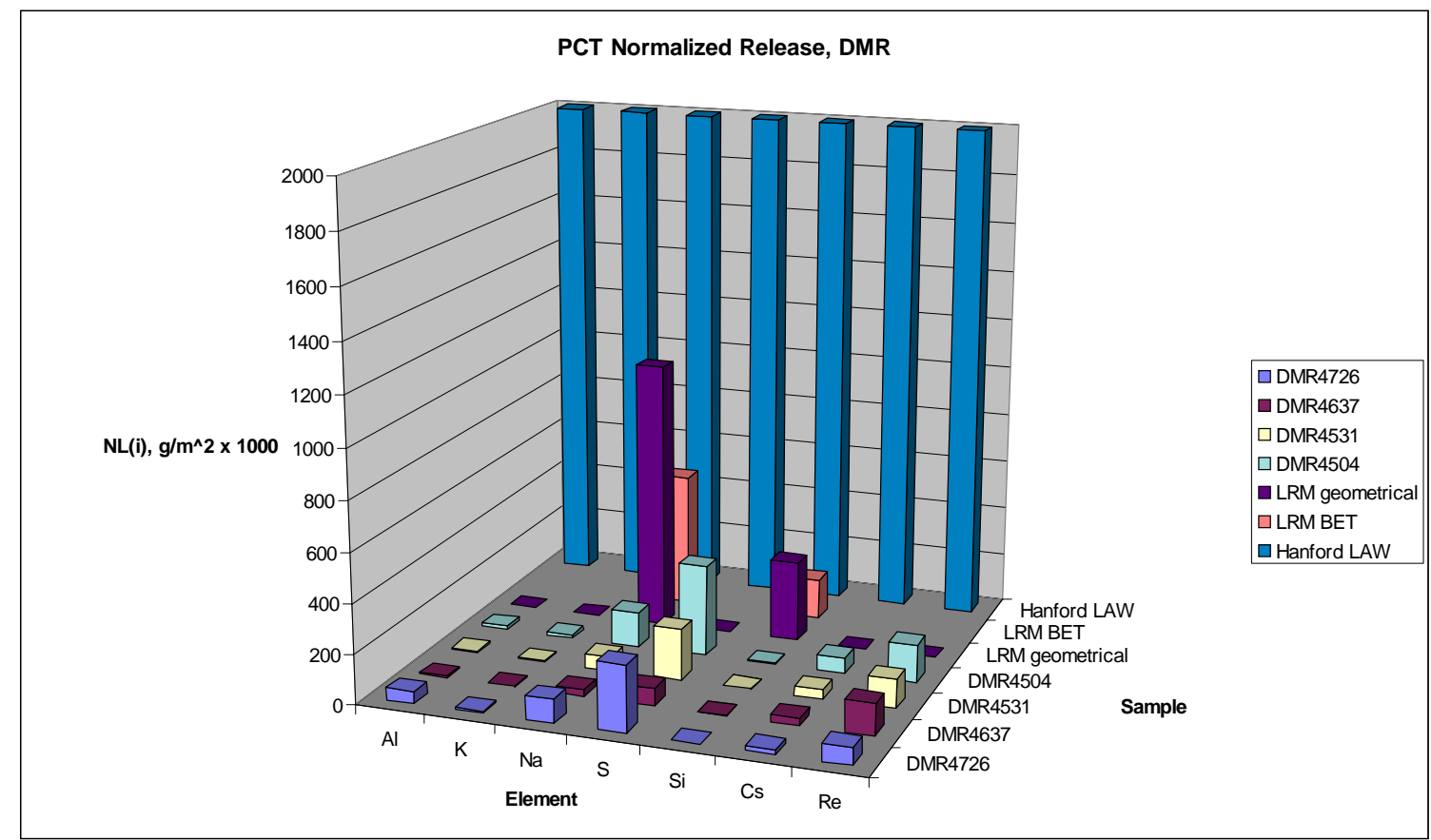

Figure 4-3. Normalized Release for DMR vs. LRM Glass and Hanford Contract LAW

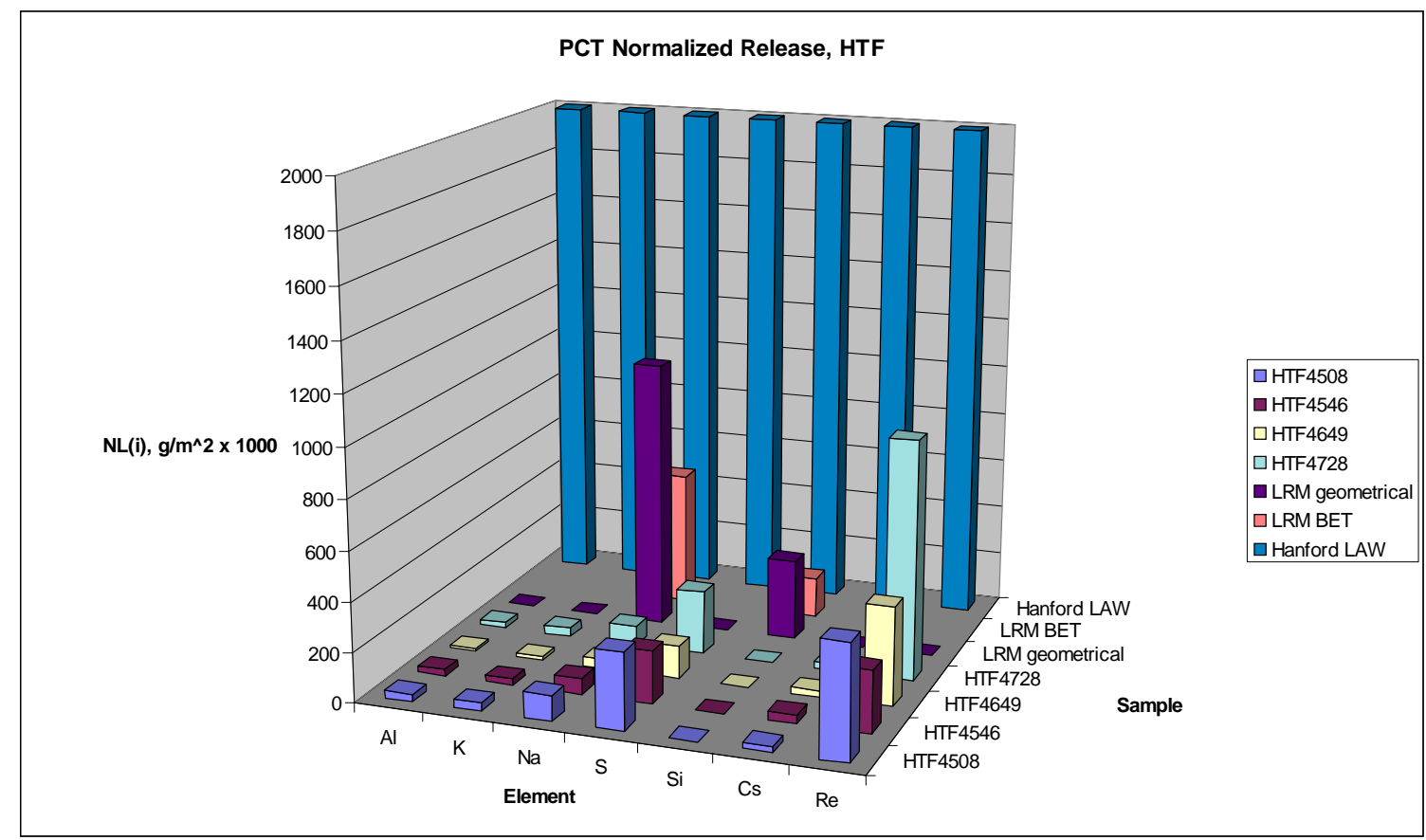

Figure 4-4. Normalized Release for HTF vs. LRM Glass and Hanford Contract LAW 


\subsubsection{Comparison of 2007 FBSR Product Durability Testing to Previous Work}

Previous durability testing of FBSR products compared data sets from various FBSR campaign SBW simulants and LAW simulants (see Table 4-1 of Pareizs et al., 2005). It is therefore instructive to also compare our recent 2007 PCT results from the Hazen 2006 SBW to previous testing. Figures 4-5 and 4-6 show the recent DMR and HTF PCT results for NL(Na) vs. NL(Al) and NL(Cs) vs. NL(Al) overlaid onto the previous comparison plots from Pareizs et al., 2005). The solid line in these figures represents the best fit of the Pareizs et al. data and the dotted lines indicate the 95\% confidence interval. Figure 4-5 indicates that all current DMR and HTF values (except for the early processing DMR 4504 and DMR 4531 points) fall within the previous fit of 0.76 slope. These 2 DMR samples not fitting the correlation contained the highest fraction of the startup bed and the beta-alumina phase $\left(\mathrm{NaAl}_{11} \mathrm{O}_{17}\right)$ shown previously in Table 4-3. Similar conclusions are drawn from the NL(Cs) vs. the NL(Al). Current DMR and HTF values are in good agreement with the correlation that shows a 1.09 slope, except for the single DMR 4726 sample.

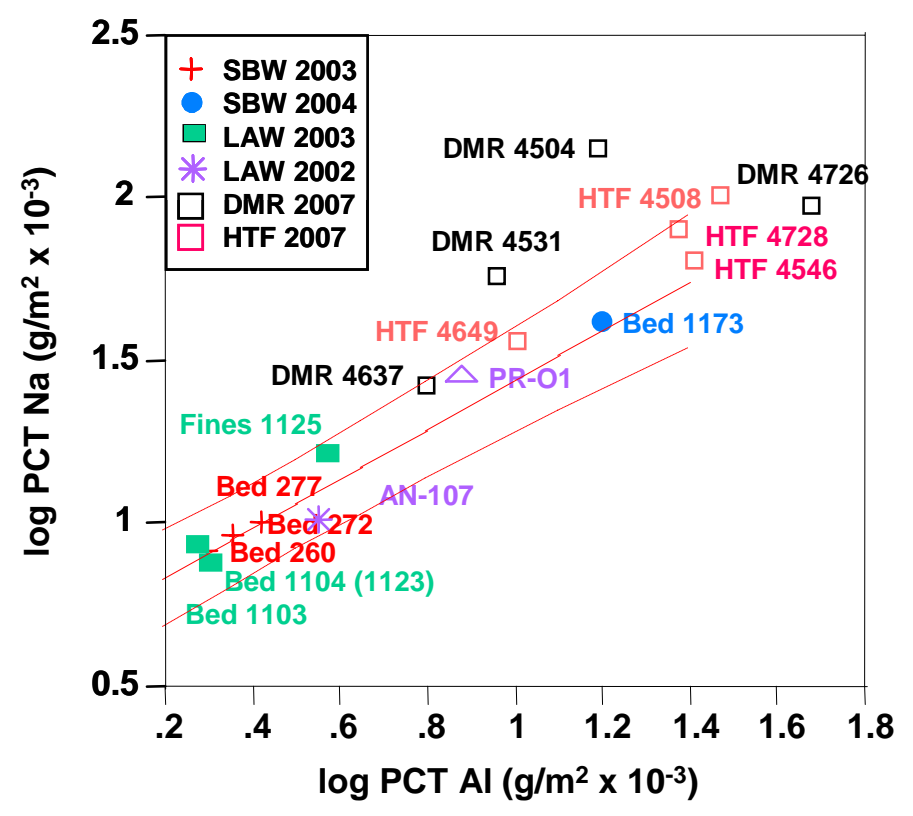

Figure 4-5. Linearity of Alkali (NL(Na)) and Alumina Released to Solution. 
WSRC-STI-2007-00319, REV. 0

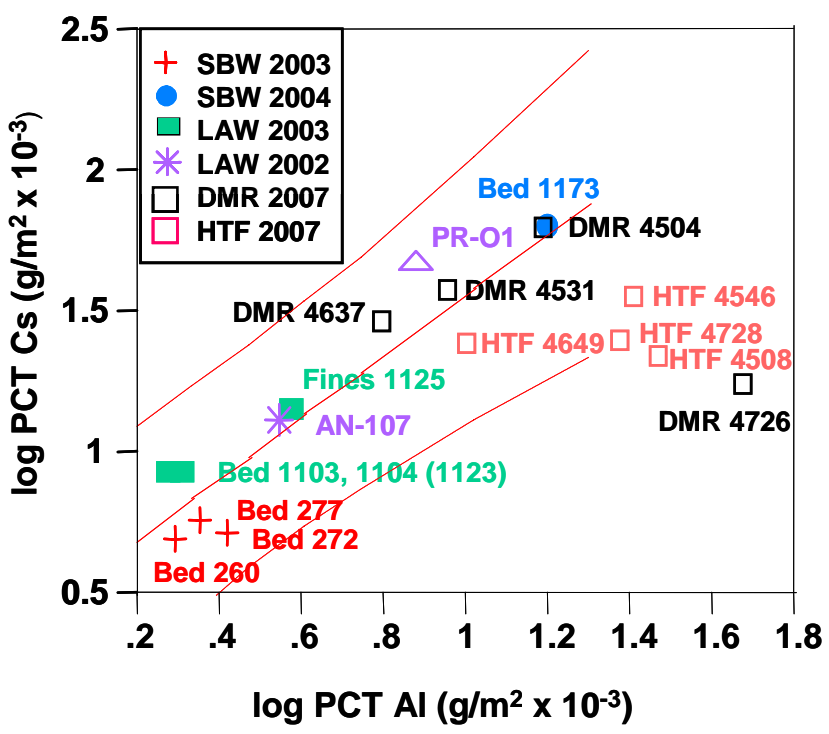

Figure 4-6. Linearity of Alkali (NL(Cs)) and Alumina Released to Solution.

Previous PCT testing indicated that $\mathrm{Re}, \mathrm{S}$ and Si released to the PCT leachate is a strong function of the leachate final $\mathrm{pH}$ as controlled by the aluminosilicate reaction mechanisms discussed in Section 5.3.1 of Pareizs et al., 2005. The plots of Figure 5-11 from Pareizs et al., 2005 have been reproduced here with the recent 2007 PCT data as shown in Figure 4-7. The PCT response for Re vs. pH for the 2007 samples shows that the HTF samples do not correlate well with previous testing and that the DMR samples are only slightly better. Similar comparisons for 2007 PCT data for $\mathrm{S}$ vs. $\mathrm{pH}$ indicate excellent correlation with previous testing. The Si values from current 2007 testing show good correlation for the DMR samples, but the HTF samples are outside the $95 \%$ confidence interval of the previous correlation. 
WSRC-STI-2007-00319, REV. 0
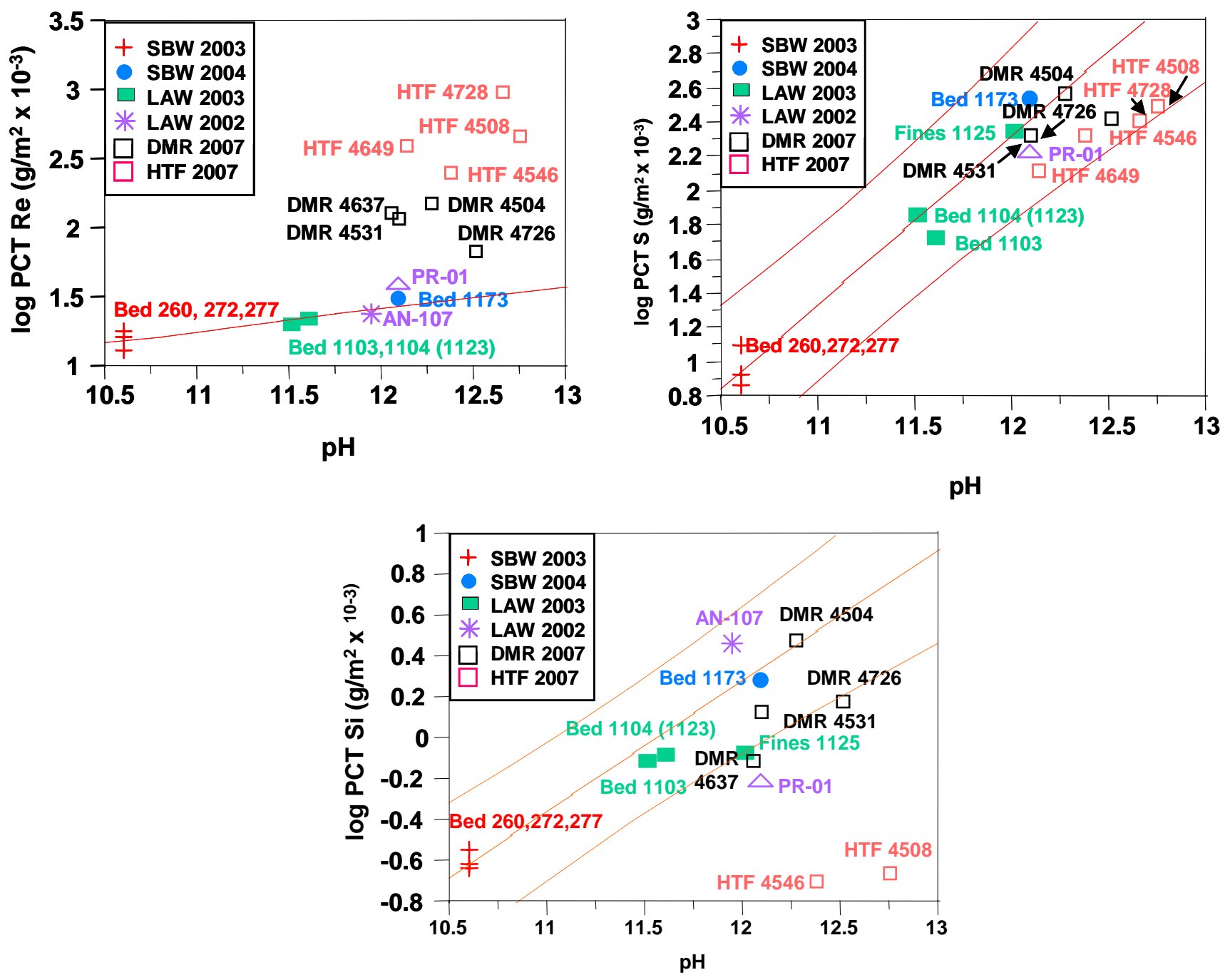

Figure 4-7. Release of Re, S, and Si to the PCT Leachates as a Function of the pH of the Final Leachate.

Previous correlations of $\mathrm{Re}, \mathrm{S}$ and $\mathrm{Si}$ that showed strong correlation to $\mathrm{pH}$ also showed correlation to each other as in Figure 5-12 of Pareizs et al, 2005. Those plots are presented below in Figure 4-8 for comparison. In all cases, the current 2007 NL(i) values for all HTF samples (Re vs. S, Re vs. Si, and S vs. Si) correlate higher than previous comparisons. The current 2007 NL(i) values for the DMR samples are all closer to the previous correlations, with the S vs. Si DMR correlation falling within the $95 \%$ confidence interval of previous testing. 
WSRC-STI-2007-00319, REV. 0
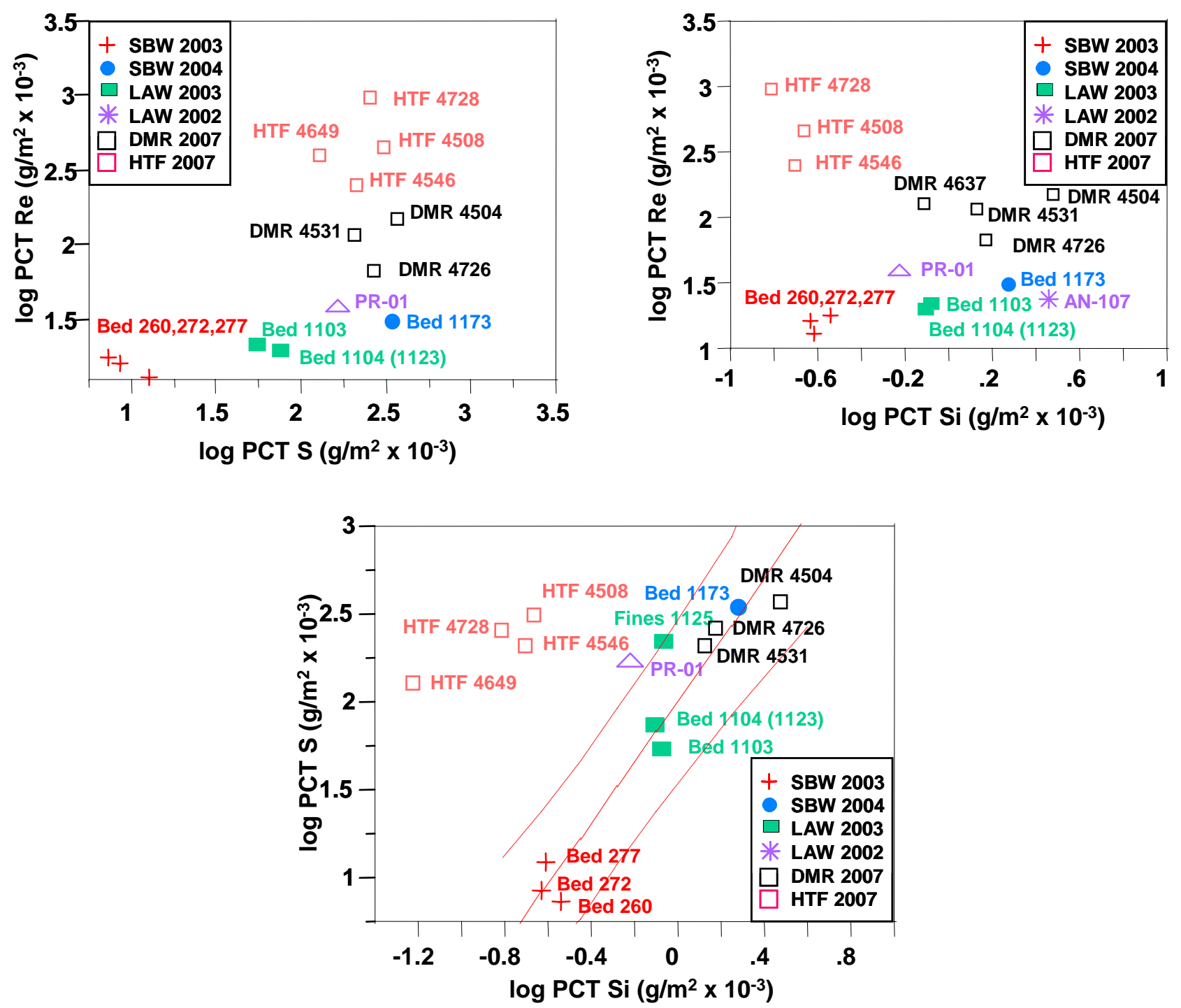

Figure 4-8. Relation of Re to S, Re to Si, and S to $\mathrm{Si}$ in the PCT Leachates of the FBSR Products Tested

\subsection{TOXICITY CHARACTERISTIC LEACHING PROCEDURE}

TCLP results for the DMR and HTF samples are presented in Table 4-9. The samples submitted for TCLP were as-received and were not sized or sieved or ashed to remove carbon. The samples met the criteria for the EPA RCRA Universal Treatment Standards for all of the constituents in the simulants. The TCLP analyses were performed for all eight RCRA metals and the additional UTS metals $\mathrm{Ni}$ and $\mathrm{Zn}$. However, only $\mathrm{Cr}, \mathrm{Pb}$ and $\mathrm{Hg}$ RCRA metals were present in the SBW simulants. A complete Analytical Report for the TCLP tests and analyses is presented in Appendix B to this report. 
WSRC-STI-2007-00319, REV. 0

Table 4-9. Toxicity Characteristic Leaching Procedure (TCLP) Results

\begin{tabular}{|c|c|c|c|c|c|c|c|c|c|c|c|c|}
\hline Element & DMR4504 & DMR4531 & DMR4637 & DMR4726 & HTF4508 & HTF4546 & HTF4649 & HTF4728 & $\begin{array}{c}\text { Reporting } \\
\text { Limit }\end{array}$ & $\begin{array}{c}\text { Method } \\
\text { Detection } \\
\text { Limit } \\
\end{array}$ & $\begin{array}{c}\text { Characteristic } \\
\text { RCRA Limits } \\
*\end{array}$ & UTS $* *$ \\
\hline & $(\mathrm{mg} / \mathrm{L})$ & $(\mathrm{mg} / \mathrm{L})$ & $(\mathrm{mg} / \mathrm{L})$ & $(\mathrm{mg} / \mathrm{L})$ & $(\mathrm{mg} / \mathrm{L})$ & $(\mathrm{mg} / \mathrm{L})$ & $(\mathrm{mg} / \mathrm{L})$ & $(\mathrm{mg} / \mathrm{L})$ & $(\mathrm{mg} / \mathrm{L})$ & $(\mathrm{mg} / \mathrm{L})$ & $(\mathrm{mg} / \mathrm{L})$ & $(\mathrm{mg} / \mathrm{L})$ \\
\hline As & $0^{\mathrm{U}}$ & $0^{\mathrm{U}}$ & $0.011^{\mathrm{J}}$ & $0.014^{\mathrm{J}}$ & $0^{\mathrm{U}}$ & $0^{\mathrm{U}}$ & $0^{\mathrm{U}}$ & $0^{\mathrm{U}}$ & 1 & 0.0086 & 5 & 5 \\
\hline $\mathrm{Ba}$ & $0.23^{\mathrm{J}}$ & $0.2^{\mathrm{J}}$ & $0.29^{\mathrm{J}}$ & $0.27^{\mathrm{J}}$ & $0.29^{\mathrm{J}}$ & $0.19^{\mathrm{J}}$ & $0.34^{\mathrm{J}}$ & $0.26^{\mathrm{J}}$ & 1 & 0.0016 & 100 & 21 \\
\hline $\mathrm{Cd}$ & $0^{\mathrm{U}}$ & $0^{\mathrm{U}}$ & $0^{\mathrm{U}}$ & $0^{\mathrm{U}}$ & $0^{\mathrm{U}}$ & $0^{\mathrm{U}}$ & $0^{\mathrm{U}}$ & $0^{\mathrm{U}}$ & 1 & 0.0022 & 1 & 0.11 \\
\hline $\mathrm{Cr}$ & $0.19^{\mathrm{J}}$ & $0.15^{\mathrm{J}}$ & $0.44^{\mathrm{J}}$ & $0.055^{\mathrm{J}}$ & $0.11^{\mathrm{J}}$ & $0.016^{\mathrm{J}}$ & $0.012^{\mathrm{J}}$ & $0^{\mathrm{U}}$ & 1 & 0.004 & 5 & 0.6 \\
\hline $\mathrm{Pb}$ & $0.12^{\mathrm{J}}$ & $0.074^{\mathrm{J}}$ & $0.2^{\mathrm{J}}$ & $0.024^{\mathrm{J}}$ & $0.02^{\mathrm{J}}$ & $0.011^{\mathrm{J}}$ & $0.013^{\mathrm{J}}$ & $0.016^{\mathrm{J}}$ & 1 & 0.006 & 5 & 0.75 \\
\hline $\mathrm{Se}$ & $0.13^{\mathrm{JB}}$ & $0.13^{\mathrm{JB}}$ & $0.12^{\mathrm{JB}}$ & $0.1^{\mathrm{JB}}$ & $0.13^{\mathrm{JB}}$ & $0.12^{\mathrm{JB}}$ & $0.11^{\mathrm{JB}}$ & $0.14^{\mathrm{JB}}$ & 1 & 0.023 & 1 & 5.7 \\
\hline $\mathrm{Ag}$ & $0^{\mathrm{U}}$ & $0^{\mathrm{U}}$ & $0^{\mathrm{U}}$ & $0^{\mathrm{U}}$ & $0^{\mathrm{U}}$ & $0^{\mathrm{U}}$ & $0^{\mathrm{U}}$ & $0^{\mathrm{U}}$ & 1 & 0.0038 & 5 & 0.14 \\
\hline $\mathrm{Hg}$ & $0^{\mathrm{U}}$ & $0^{\mathrm{U}}$ & $0^{\mathrm{U}}$ & $0^{\mathrm{U}}$ & $0^{\mathrm{U}}$ & $0^{\mathrm{U}}$ & $0^{\mathrm{U}}$ & $0^{\mathrm{U}}$ & 0.02 & 0.00086 & 0.2 & 0.025 \\
\hline $\mathrm{Ni}$ & $0.37^{\mathrm{J}}$ & $0.34^{\mathrm{J}}$ & $0.37^{\mathrm{J}}$ & $0.046^{\mathrm{J}}$ & $0.0044^{\mathrm{J}}$ & $0.01^{\mathrm{J}}$ & $0.005^{\mathrm{J}}$ & $0^{\mathrm{U}}$ & 20 & 0.0016 & - & 11 \\
\hline $\mathrm{Zn}$ & $0.4^{\mathrm{J}}$ & $0.57^{\mathrm{J}}$ & $0.76^{\mathrm{J}}$ & $0.1^{\mathrm{JB}}$ & $0.018^{\mathrm{JB}}$ & $0.017^{\mathrm{JB}}$ & $0.019^{\mathrm{JB}}$ & $0^{\mathrm{U}}$ & 40 & 0.012 & - & 4.3 \\
\hline
\end{tabular}

*40 CFR 261.24 - “Toxicity Characteristic'

**40 CFR 268.48 - "Universal Treatment Standards (UTS)"

$\mathrm{U}=$ results less than $\mathrm{MDL}$, or " $\mathrm{U}$ " flag

$\mathrm{J}=$ results below Reporting Limit but above the MDL, or "J" flag

$\mathrm{JB}=$ results below Reporting Limit but above the MDL, and detectable amounts in blank ( $\mathrm{Se}=0.078 \mathrm{mg} / \mathrm{L}$ and $\mathrm{Zn}=0.024 \mathrm{mg} / \mathrm{L})$, or "JB" flag 


\subsection{QUALITY ASSURANCE}

This work was completed in accordance with WSRC and SRNL quality assurance requirements. The waste form characterization and testing was conducted in accordance with DOE/RW-0214, DOE/RW-0333P, and ASME NQA-1 based quality assurance programs.

The data is recorded in the following notebook: WSRC-NB-2007-00096. 


\subsection{CONCLUSIONS}

The FBSR process was performed on INTEC simulated SBW in a recent pilot-scale demonstration that produced representative DMR and HTF samples. These powdered samples were analyzed and leach tested in this task. The following conclusions can be drawn from the testing.

- Elemental compositional analysis of the DMR and HTF samples indicates that they contain primarily $\mathrm{Al}, \mathrm{Si}$ and $\mathrm{Na}$, with $>1 \mathrm{wt} \%$ of $\mathrm{Ca}, \mathrm{Fe}$ and $\mathrm{K}$ also present. No detectable mercury was observed. The DMR samples all contained less than $1 \%$ carbon. The HTF samples containing carbon in the range of $13-26 \mathrm{wt} \% \mathrm{had}$ detectable nitrite anion levels and their REDOX values show that they were completely reduced with $\mathrm{Fe}^{2+} / \mathrm{Fe}_{\text {total }}$ ratios of one.

- Crystalline phases observed in the coarse-sieved DMR samples support increasing alumina bed turnover in the FBSR processing, with the final two DMR samples comprised mostly of nepheline group mineral phases. All of the HTF samples contained nepheline.

- $\quad$ PCT durability testing of the DMR and HTF samples along with appropriate HLW and LAW reference or benchmark glasses shows that the FBSR products are orders of magnitude more durable than the HLW EA glass, and at least 2X more durable than the current Hanford RPP WTP limit for LAW product disposal.

- REDOX results indicate that the HTF samples were overly reduced to form the desired Re host phase. This likely influenced the durability of the Re and S oxide components in these samples, contributing to slightly higher normalized release when compared to other components in the HTF products.

- The TCLP results performed on the FBSR samples by an independent off-site EPAcertified laboratory indicate that these products meet the criteria for the EPA RCRA Universal Treatment Standards for all of the constituents $(\mathrm{Cr}, \mathrm{Pb}$ and $\mathrm{Hg})$ in the simulants. 


\section{ACKNOWLEDGEMENTS}

The authors wish to acknowledge many colleagues who contributed to the completion of this effort. Sherry Vissage prepared the samples for all compositional analysis and leach testing. Dave Best and Pat Toole performed the DMR/HTF chemical analyses and REDOX using ICP-AES in the SRNL Process Science Analytical Laboratory (PSAL). Several different SRNL Analytical Development personnel contributed. Art Jurgensen and David Missimer performed the XRD analyses. Curtis Johnson directed the ICP-MS analyses and performed the $\mathrm{Hg}$ dissolutions and analyses.

The authors would also like to thank Kevin Ryan of WGI and Arlin L. Olson, Idaho Cleanup Project, CH2M WG Idaho, LLC for technical advice and fruitful discussions regarding this work.

Finally, the authors would like to thank Mimi Jones for her support in the technical editing of this report. 


\section{Distribution:}

Savannah River National Laboratory

J. E. Marra, 773-A

R. E. Edwards, 773-A

D. A. Crowley, 999-W

A. B. Barnes, 999-W

C. C. Herman, 999-W

D. B. Burns, 786-5A

N. E. Bibler, 773-A

C. M. Jantzen, 773-A

C. L. Crawford, 773-41A

J. C. Marra, 773-42A

J. R. Harbour, 773-42A

G. G. Wicks, 773-A

J. M. Pareizs, 773-A

D. K. Peeler, 999-W

W. E. Daniel, 999-W

M. R. Williams, 786-5A

P. R. Burket, 773-42A

M. E. Smith, 999-W

A. D. Cozzi, 999-W

\section{$\underline{\text { THOR Treatment Technologies }}{ }^{\circledR}$ :}

Kevin Ryan, kevin.ryan@wgint.com

Brad Mason, brad.mason@wgint.com

Brent Evans, brent.evans@wgint.com

Vishal Vora, vishal.vora@wgint.com

Idaho Cleanup Project, CH2M WG Idaho, LLC:

Arlin Olson, Arlin.Olson@icp.doe.gov

Scott Roesener, W.Roesener@icp.doe.gov

John Tadlock, John.Tadlock@icp.doe.gov

Eldredge Engineering:

Brad Eldredge, brad@eldeng.com

U.S. Department of Energy:

Joel Case, CASEJT@ID.DOE.GOV

Bill Owca, OWCAWA@ID.DOE.GOV 
WSRC-STI-2007-00319, REV. 0

\subsection{REFERENCES}

ASTM C1285. Annual Book of ASTM Standards, Vol. 12.01: Standard Test Methods for Determining Chemical Durability of Nuclear, Hazardous, and Mixed Waste Glasses and Multiphase Glass Ceramics: The Product Consistency Test (PCT), (2002).

Burket, P.R., Marra, J.C., Pareizs, J.M. and Jantzen, C.M., "Evaluation of Fluidized Bed Steam Reforming (FBSR) Technology for Sodium Bearing Wastes From Idaho and Hanford Using the Bench-Top Steam Reformer (BSR)," U.S. DOE Report WSRC-TR2004-00560 (Jan 2005).

Ebert, W. L. and S. F. Wolf, “An Interlaboratory Study of a Standard Glass for Acceptance Testing of Low-activity Waste Glass,” J. of Nuc. Materials, 282, pp. 112-124, 2000.

Federal Register, Vol. 70, No. 148, August 3, 2005 / Notices, pp 44598 - 44600.

Jantzen, C. M. et al., “Characterization of the Defense Waste Processing Facility (DWPF) Environmental Assessment (EA) Glass Standard Reference Material," US DOE Report WSRC-TR-92-346, Rev. 1, WSRC, Aiken, SC, 1993.

Jantzen, C. M. et al., “Process/Product Models for the Defense Waste Processing Facility (DWPF): Part II. Appendix G.,” US DOE Report WSRC-TR-93-0672, p. 464, 1995.

Jantzen, C.M., Pareizs, J.M., Lorier, T.H. and Marra, J.C. "Durability Testing of Fluidized Bed Steam Reforming (FBSR) Products,” Ceramic Transactions V. 176, 121-137 (2006a).

Jantzen, C.M., Lorier, T.H., Marra, J.C., and Pareizs, J.M., “Durability Testing of Fluidized Bed Steam Reforming (FBSR) Waste Forms” WM06 Paper \#6373 (February 2006b).

Jantzen, C. M., "Fluidized Bed Steam Reformer (FBSR) Product: Monolith Formation and Characterization,” U.S. DOE Report WSRC-STI-2006-00033, Rev. 0, (June 2006c).

Land Disposal Restrictions. Code of Federal Regulations, Part 268, Title 40, 2004.

Lorier, T.H., Pareizs, J.M. and Jantzen, C.M., "Single-Pass Flow Through (SPFT) Testing of Fluidized Bed Steam Reforming (FBSR) Waste Forms," U.S. DOE Report WSRC-TR2005-00124 (October 2005).

Mason, J. B. et al., THOR Treatment Technologies, LLC and B. Landman, CH2M WG Idaho, LLC, "Steam Reforming Application for Treatment of DOE Sodium Bearing Tank Wastes at Idaho National Laboratory for Idaho Cleanup Project," Waste Management '06, Tucson, AZ, 2006. 
Office of Solid Waste, US EPA. TCLP Method 1311, Test Methods for Evaluating Solid Waste; Physical/Chemical Methods, Third Edition; EPA 530/SW-846; National Technical Information Service: Washington, DC, 1986.

Pareizs, J. M., C. M. Jantzen and T. H. Lorier, “Durability Testing of Fluidized Bed Steam Reformer (FBSR) Waste Forms for High Sodium Wastes at Hanford and Idaho (U),” U.S. DOE Report WSRC-TR-2005-00102 (May 2005).

Pilot Plant Report for Treating SBW Simulants: Mineralizing Flowsheet, Report Number 28266-RT-002, Rev. 0, THOR Treatment Technologies ${ }^{\text {sm }}$, July 2007.

Soelberg, N. R. et al., "Phase 2 THOR Steam Reforming Tests for Sodium-Bearing Waste Treatment," INEEL/EXT-04-01493, Idaho National Engineering and Environmental Lab, Bechtel BWXT Idaho, LLC, January 2004a.

Soelberg, N. R. et al., "Phase 2 TWR Steam Reforming Tests for Sodium-Bearing Waste Treatment," INEEL/EXT-04-01494, Idaho National Engineering and Environmental Lab, Bechtel BWXT Idaho, LLC, January 2004b.

WAPS-Waste Acceptance Product Specifications for Vitrified High Level Waste Forms, U. S. Department of Energy, Office of Environmental Restoration and Waste Management, USDOE Document EM-WAPS, Washington, DC (February, 2003).

WTP Contract, Section C, Statement of Work, Contract No. DE-AC27-01RV14136, Modification No. M076, 2007 
WSRC-STI-2007-00319, REV. 0

APPENDIX A - XRD SPECTRA OF DMR AND HTF SAMPLES

\begin{tabular}{|l|}
\hline DMR 4504 \\
\hline DMR 4531 \\
\hline DMR 4637 \\
\hline DMR 4726 \\
\hline \\
\hline HTF 4508 \\
\hline HTF 4546 \\
\hline HTF 4649 \\
\hline HTF 4728 \\
\hline
\end{tabular}


WSRC-STI-2007-00319, REV. 0

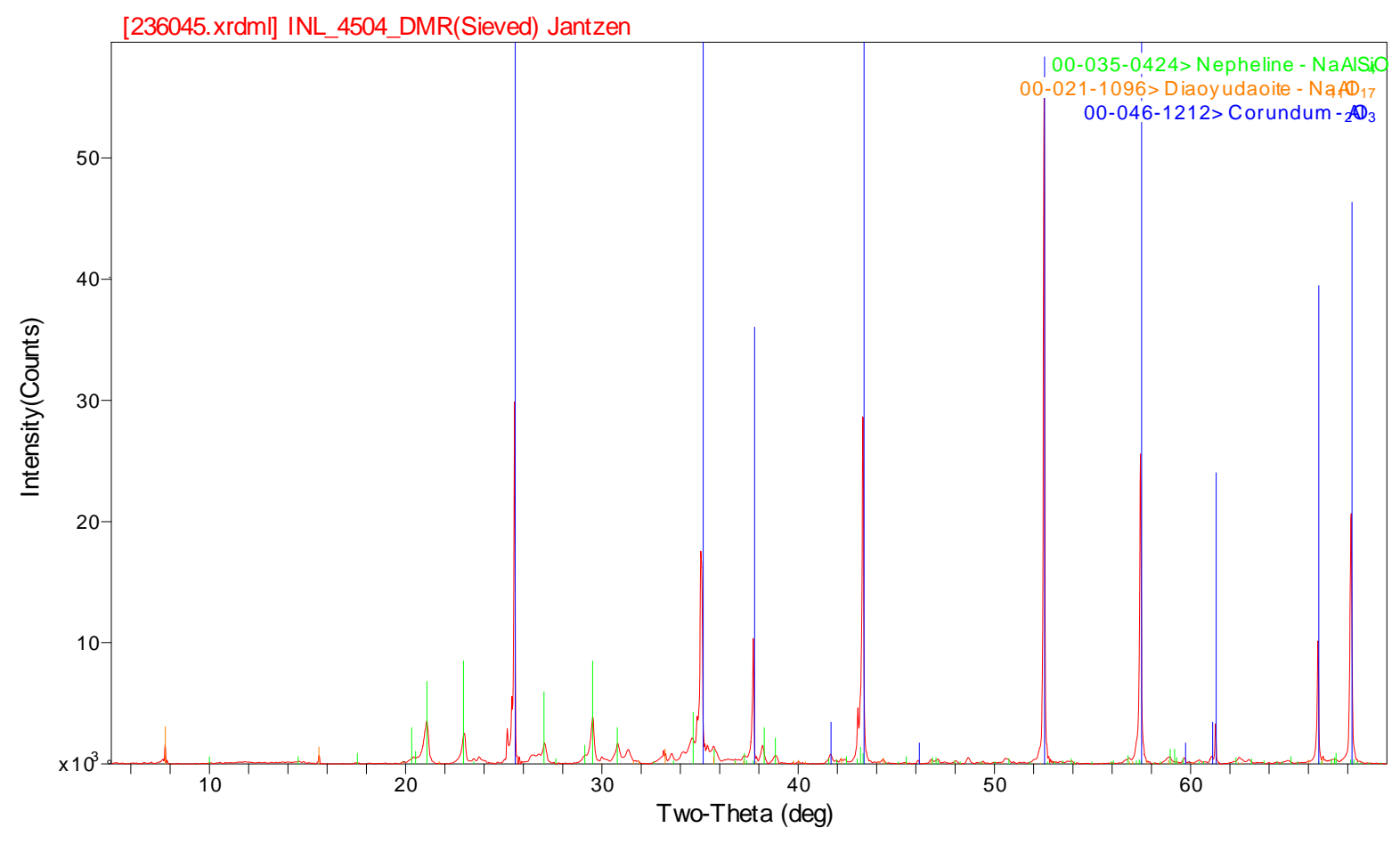

Figure A- 1. INL 4504 DMR (Sieved) 
WSRC-STI-2007-00319, REV. 0

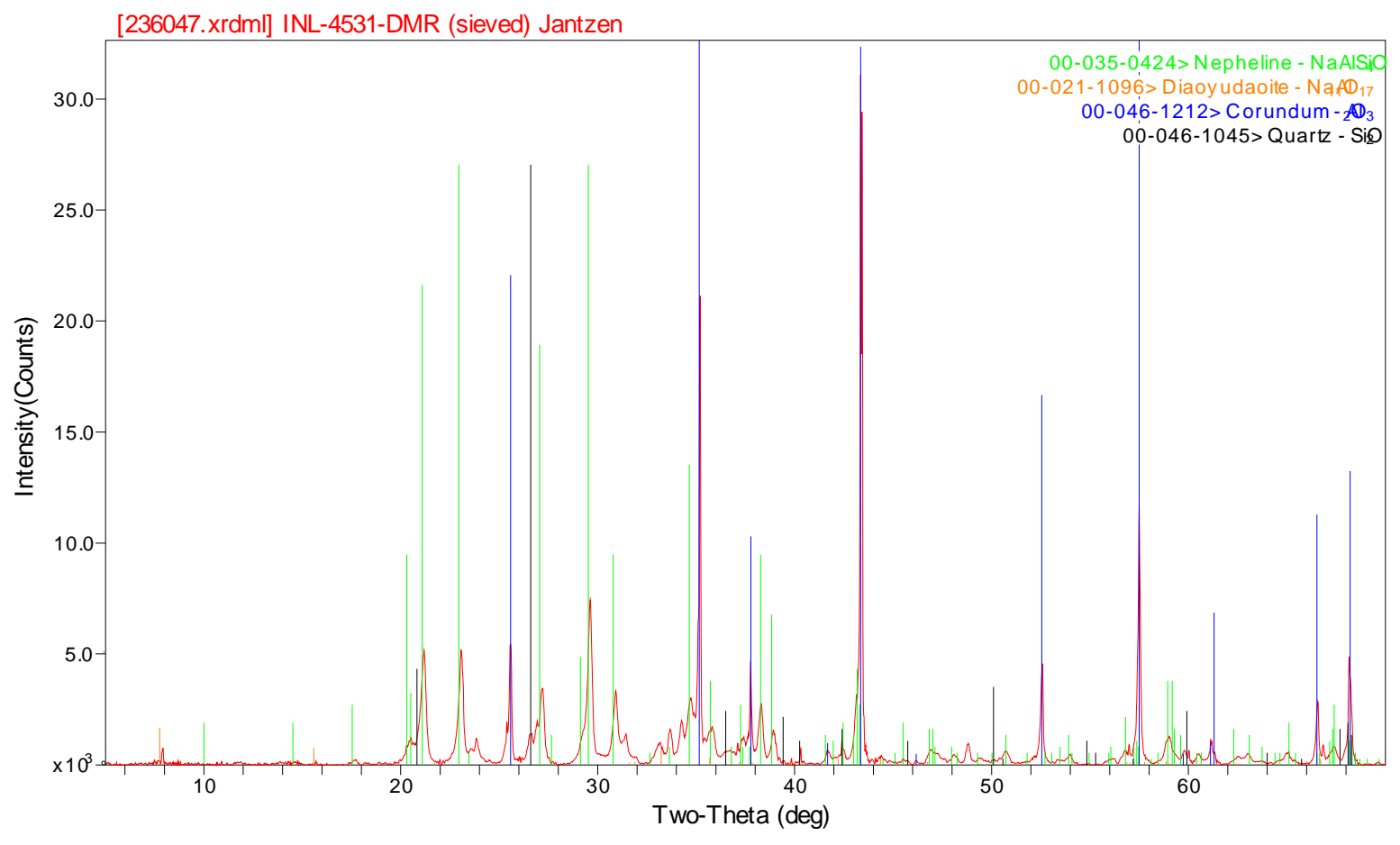

Figure A- 2. INL 4531 DMR (Sieved) 
WSRC-STI-2007-00319, REV. 0

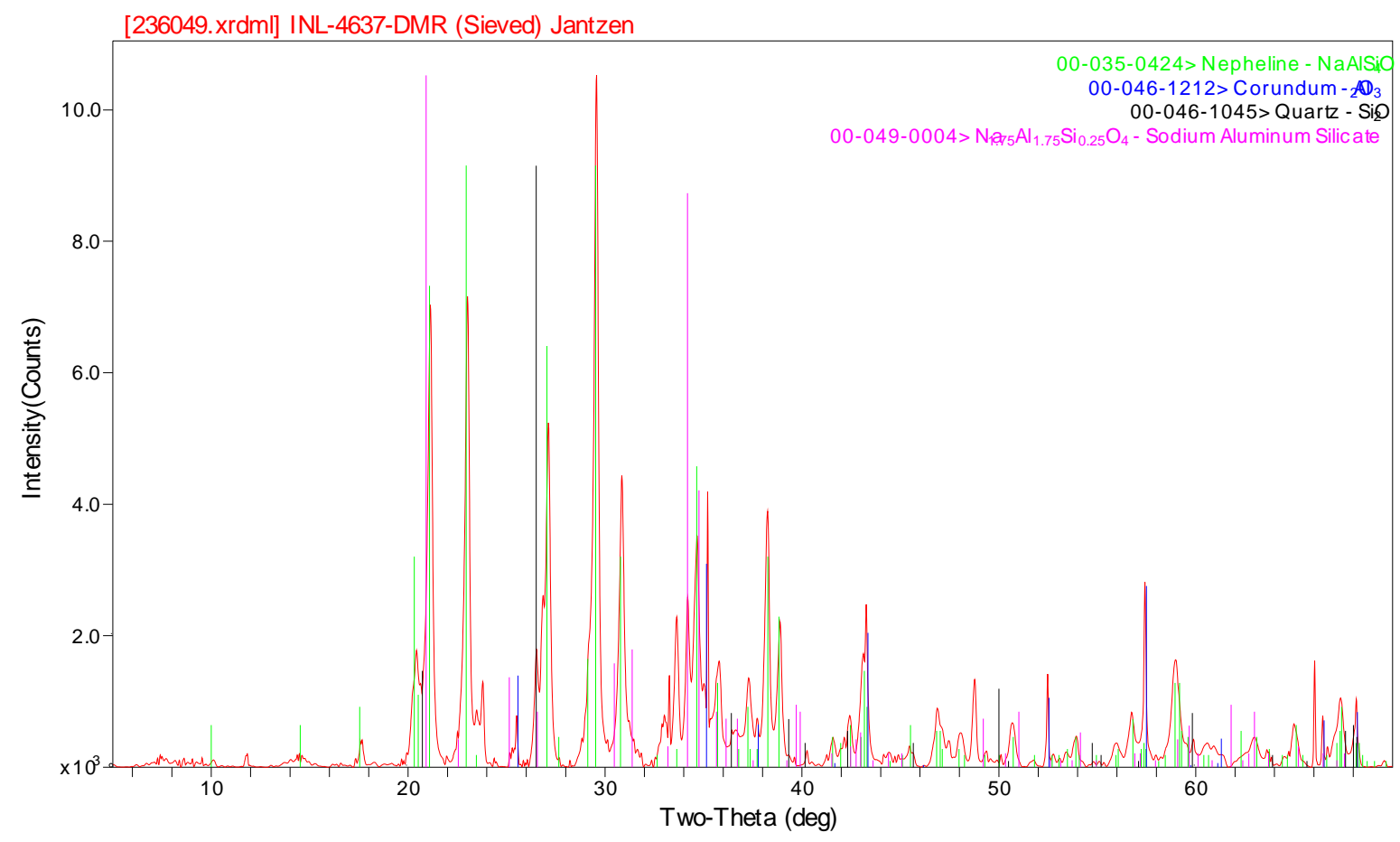

Figure A- 3. INL-4637-DMR (Sieved) 
WSRC-STI-2007-00319, REV. 0

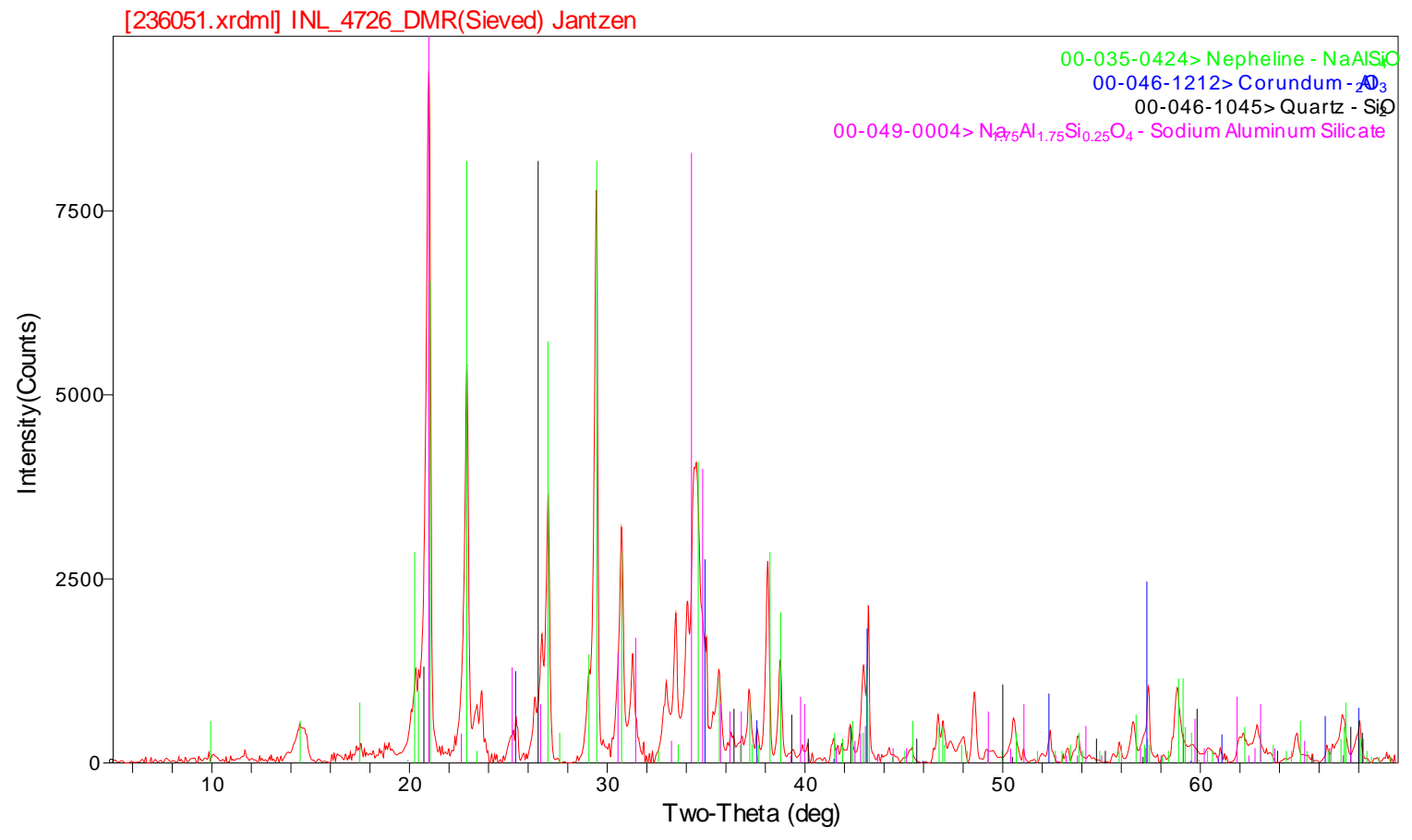

Figure A- 4. INL-4726-DMR (Sieved) 
WSRC-STI-2007-00319, REV. 0

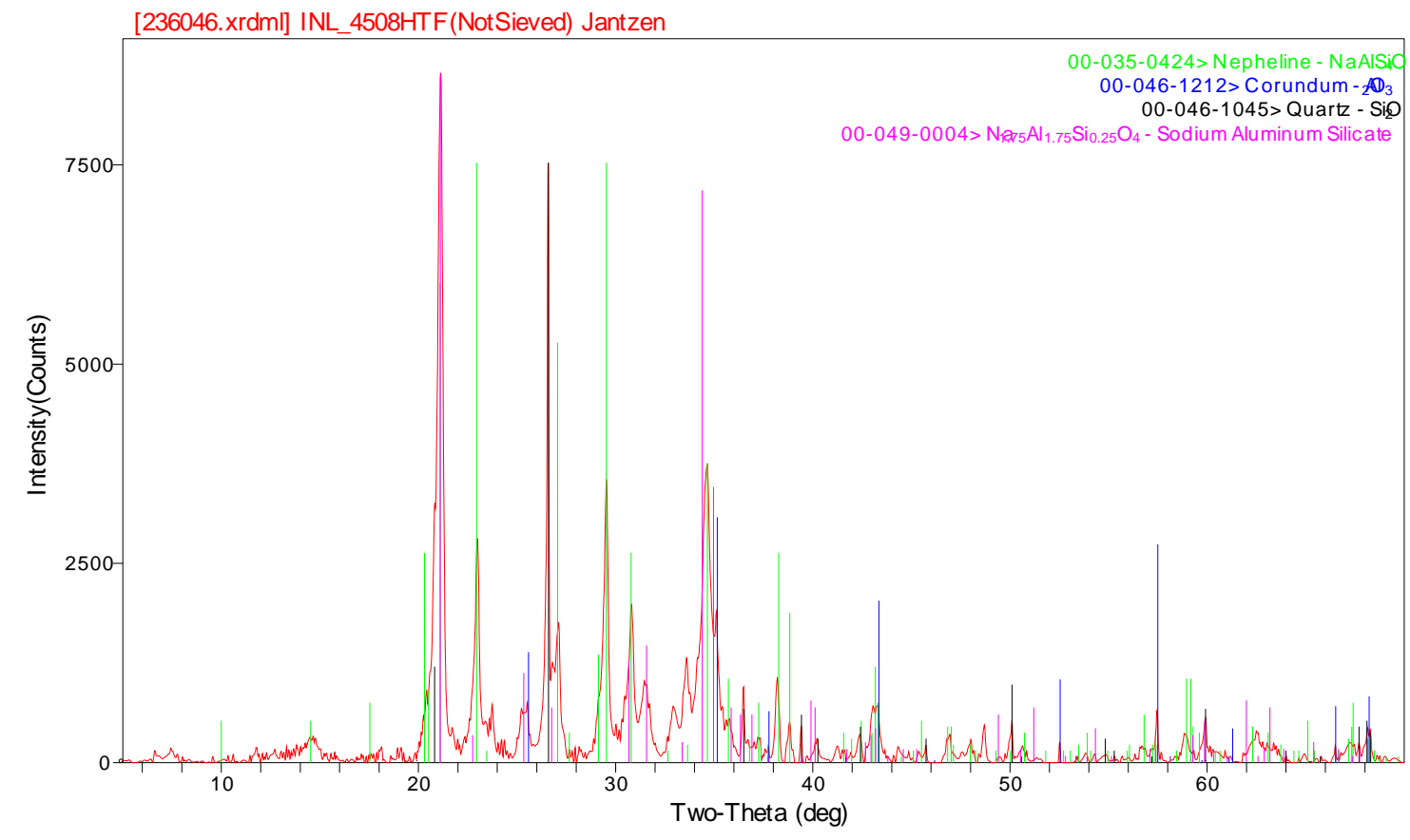

Figure A- 5. INL-4508-HTF (Not Sieved) 
WSRC-STI-2007-00319, REV. 0

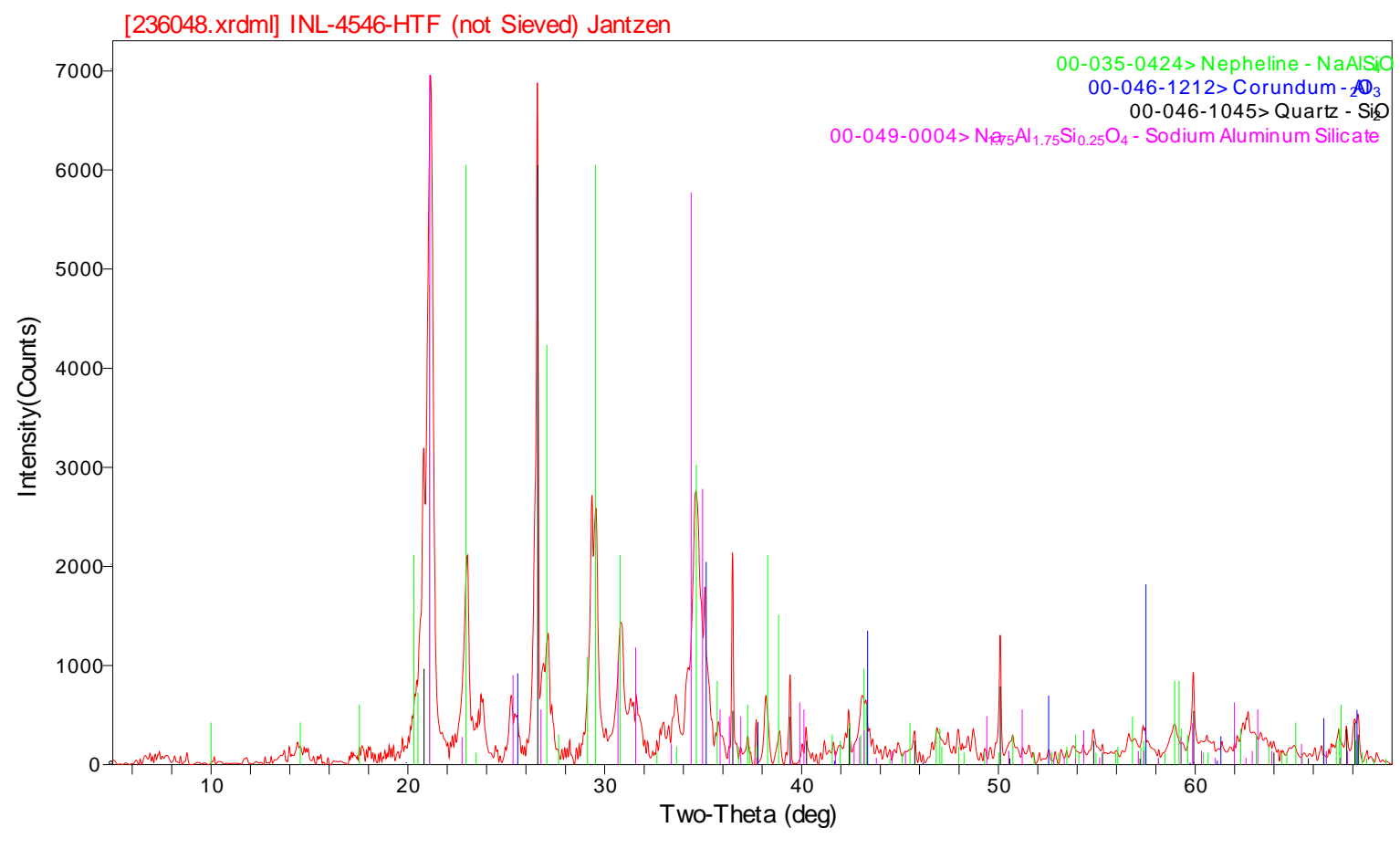

Figure A- 6. INL-4546-HTF (Not Sieved) 
WSRC-STI-2007-00319, REV. 0

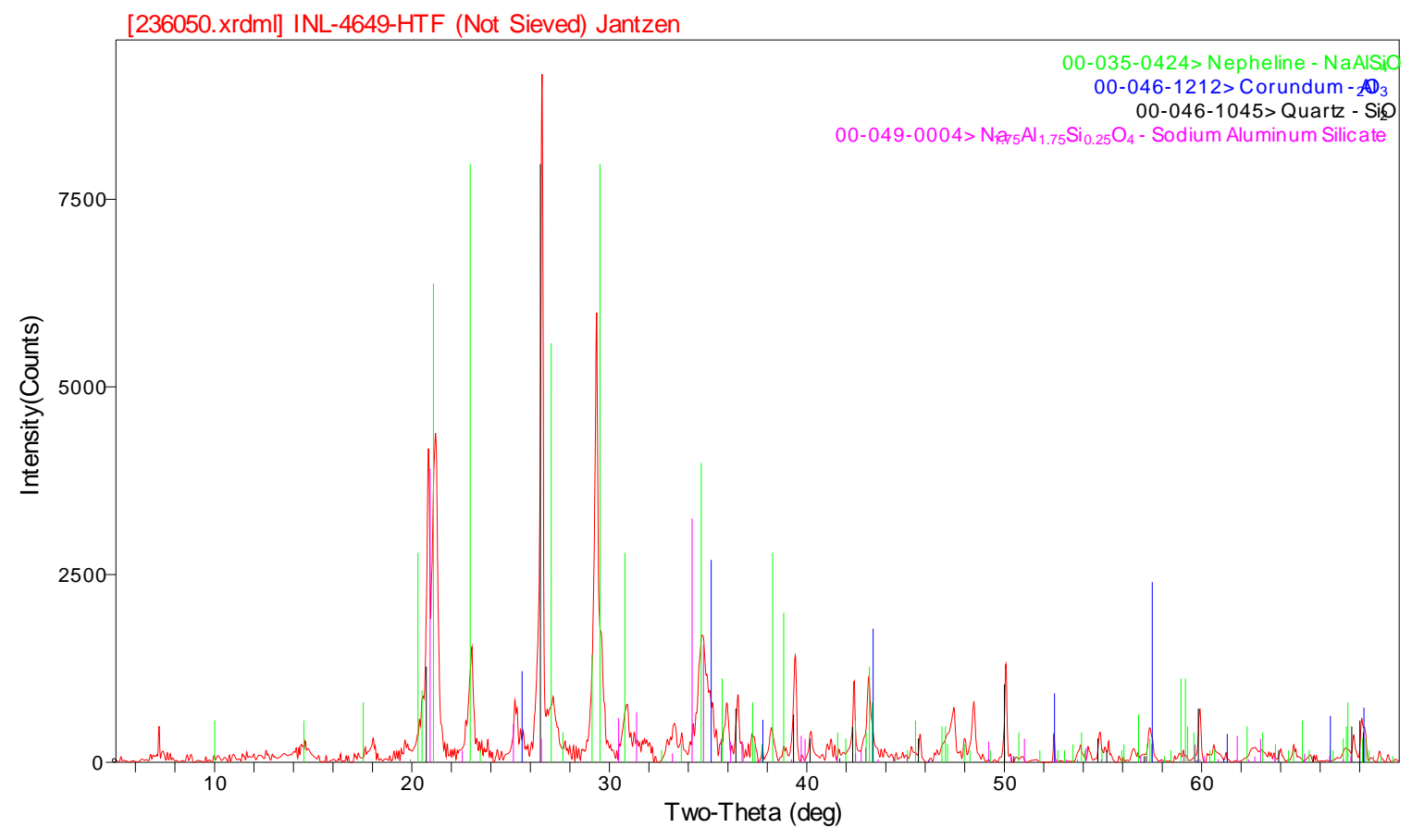

Figure A- 7. INL-4649-HTF (Not Sieved) 
WSRC-STI-2007-00319, REV. 0

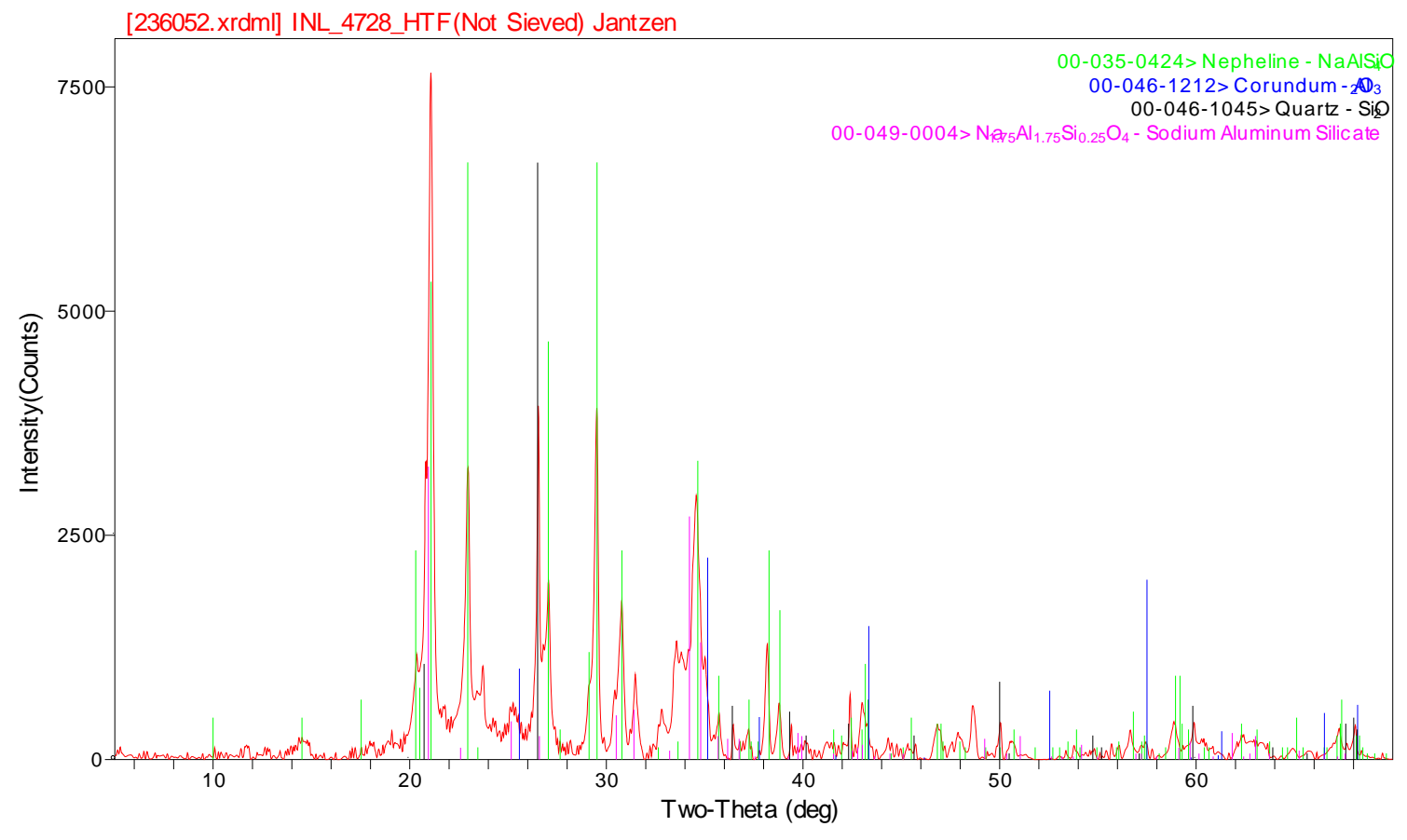

Figure A- 8. INL-4728-HTF (Not Sieved) 


\section{APPENDIX B - ACCURA REPORT}

Analytical Report for: Washington Savannah River Co.

Project Name: 07079

Project ID: 07079

Project Manager: Larry Dewitt (WSRC)

Project Location: South Carolina

Lab. Work Order \#: 11733

April 12, 2007

ACCURA Analytical Laboratory, Inc.

6017 Financial Drive, Norcross, GA 30071

The entire report from ACCURA Analytical Laboratory, Inc. follows. 


\title{
Analytical Report for:
}

\section{Washington Savannah River Co.}

\author{
Project Name : 07079 \\ Project ID : 07079 \\ Project Manager: Larry Dewitt \\ Project Location : South Carolina \\ Lab. Work Order \# : 11733

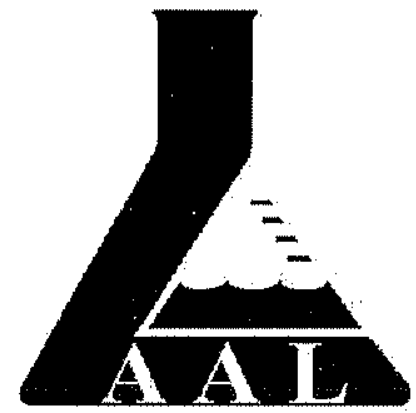

April 12, 2007

ACCURA Analytical Laboratory, Inc.

6017 Financial Drive Norcross, GA 30071

Phone: 770-449-8800 Fax: 770-449-5477 


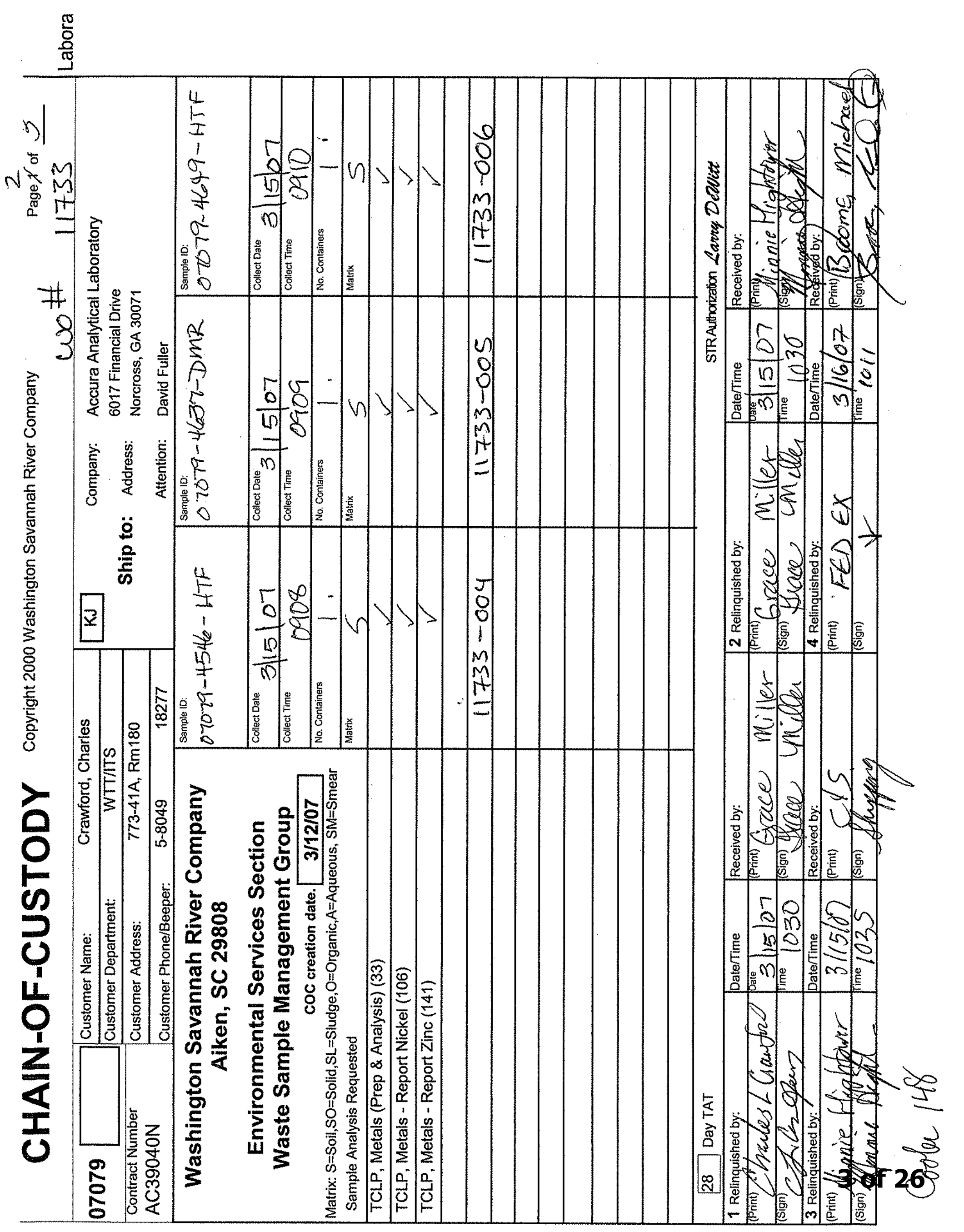


$\frac{\mathbb{8}}{8}$

$\begin{array}{ccc}m & m \\ m & M \\ 5 & = \\ 0 & \\ \pm \\ 0 \\ 3 \\ 3\end{array}$

율 尽

离 要

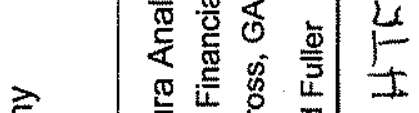

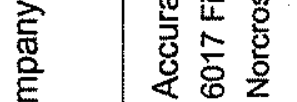

응

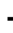

实

$\sum_{0}^{\infty}$

齐

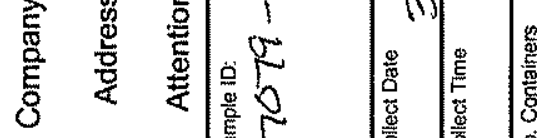

$\ddot{0}$

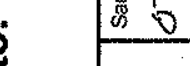

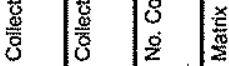

एक

$\underline{x}$<smiles>CC1(C)C2CCCC1C2</smiles>

$\sqrt{\frac{1}{2}} \frac{1}{2}$

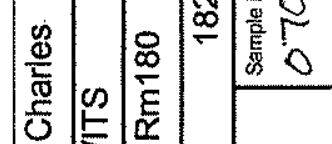

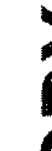

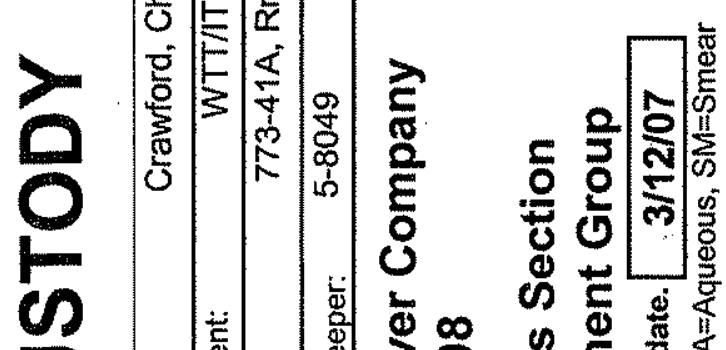

.

离部总

要

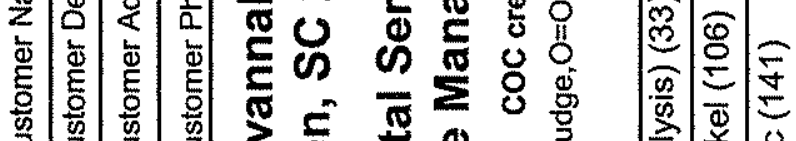

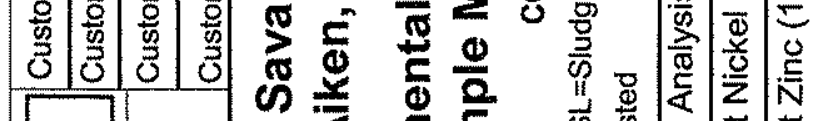

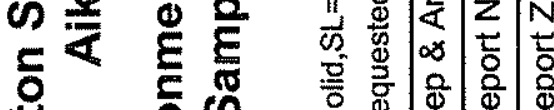

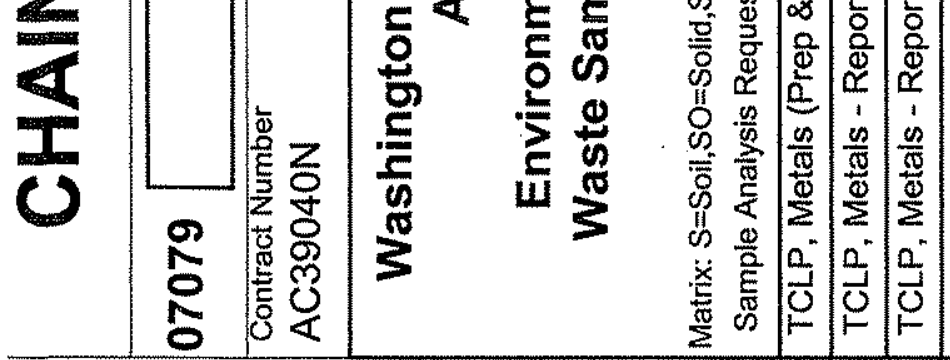

$\infty$

0

m

M

$=$

N

1

$m$

it

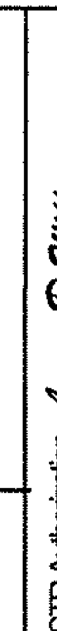

a

पे
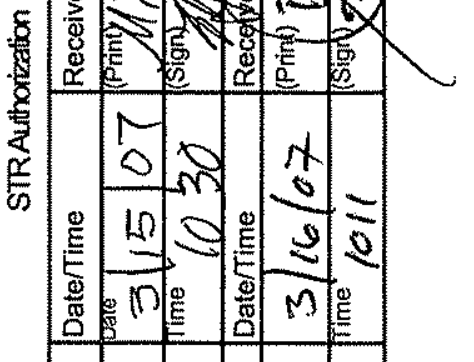

至

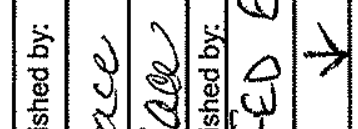

惫

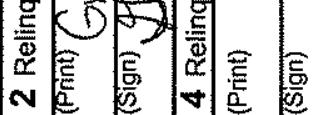
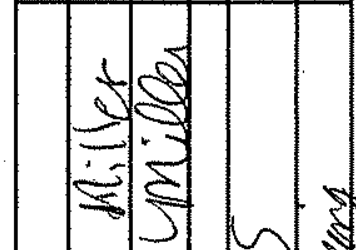

ลे

20

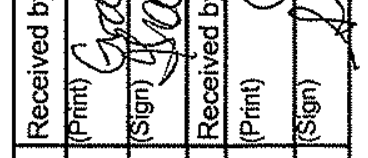

定

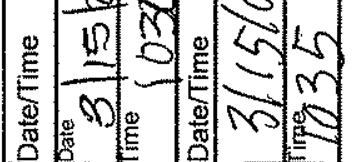

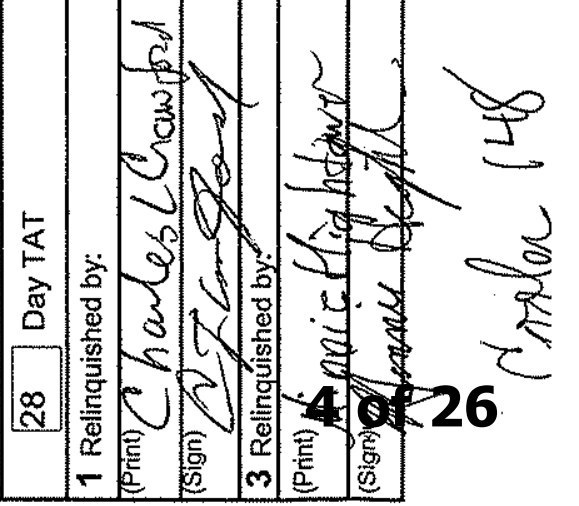


SAMPLE RECEIPT CHECKLIST VERSION 6

Client Project Name:
Client Code: 2321

AAL Project Mgr:

ACCURA Work Order\#:
DCF

1733

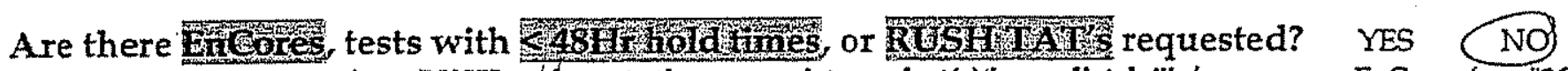

If XES, you must communicate RUSH andyses to the appropriate analyst(s) immediately!!! / or preserve EnCores (see \#16 bolow)!!! Preliminary Examination: Initials:_ $3 \mathrm{~m}$ Date received: $3 / 16 / 07$ Date cooler was opened: $3 / 16 / 02$

1. Did cooler/package come with a shipping slip (airbill, Etc.)?

If YES, enter carrier name and airbill number here: Fed Ex - $7916 \quad 5185 \quad 5227$

Describe type of packing in cooler: Babble wra / Ice

*****If cooler was hand delivered, CIRCLE HERE skip to item $\# 5^{* * * * *}$

2. Were custody seals on outside of cooler?

If YES, how many:_____seal dated:________ seal name:

3. Were custody seals unbroken and intact at the date and time of arrival?

YES

NO

3. Were custody seals unbroken and intact at the date and the of arival?

4. Were custody papers sealed in a plastic bag to prevent damage to chain of custody?

YES

(NTA)

NO

5. If required, was enough ice used? (Internal cooler temperature, $4 c$ )

(1BS

N/A

NO

6. Did you sign custody papers in the appropriate place?

YES

NO

7. Was project identifiable from custody papers?

बतs

If YES, enter project name at the top.

Complete project file with green sheet, proper file tag, and shipping documentation. Line up samples following chain. Complete Container

Receipt Verification form (include extra containers for dissolved metals filtrates). Complete login in XENCO and generate AAL ID Labels.

8. Did all containers arrive unbroken and were labels in good condition?

YES NO

9. Were custody papers filled out properly and did all labels agree with custody papers?

YES

NO

10. Were correct containers and sufficient amount of sample sent for the test indicated?

NO

11. All samples collected within three days of date received for these analyses

(Reactive Cn \& S, Solids in H2O, Sulfide, Sulfite, !ALL! Extractable Organic Waters)?

If NO, coordinate with the project manager to ensure that no samples go out of hold!!!!

12. No residual chlorine found in waters for these analyses:

(Cyaride, PAH, SVOC, Pesticides, PCB's, Herbicides)?

Checked by:

YES (Initials)

13. Were samples properly chemically preserved, if required, upon receipt?

YES

YES

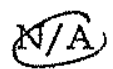

NO

(For example: $\mathrm{pH}$ checked for waters for all Metals, Wet Chemistry, Pesticides, PCB's, Herbicides, and

VOC/BTEX samples submitted with HCL for waters and in either. Encore samplers or NaHSO labeled vials for soils) Preservation checked by: (Initials)

14. Were air bubbles ( $>1 / 4$ inch) absent in VOC/BTEX samples?

YES

बIA

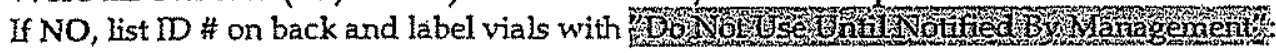

15. If there are samples for dissolved metals, were they field filtered?

YES

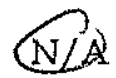

If NO, list date and time samples were filtered and preserved in lab:

16. Were Encore samplers included?

If YES, date and time preserved with $\mathrm{NaHSO}_{4}$ :

By whom:

17. Does this submittal contain soil $\mathrm{NaHSO}_{4}$ vials for BTEX/GRO/VOC'S?

If YES, vials weighed by and entered into vial database by:

18. Initials of laboratory personnel responsible for labeling laboratory I.D. numbers on containers:

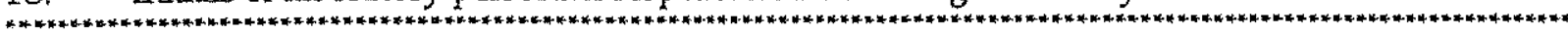

Keep samples and chain out. Before moving samples to their appropriate location, another person must review the entire project ensuring that information on the AAL WD Barcode label matches the container label, and that all information is consistent with the chain.

Final check and samples logged to locations by:

(Initials)

19. Was it necessary to call the assigned project manager in order to proceed with login? YES If YES, give details on the back of this form.

20. Who was called?

Project Mgr. Review:

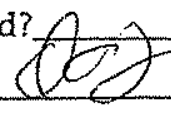

(Initials) $3 / 19 / 07$

Date/Time:

(Date) 
ACCURA ANALYTICAL LABORATORY, INC. SAMPLE RECEIPT VARIANCE FORM

\section{Item \# Discrepancies Noted:}

Item\# Actions Taken: 
Date: $3 / 16 / 07$ Time: 1600hr

Survey Type: Sample Inspection and Receipt

Client: 2321

Client Job/wo \#07079 wo\#11733

Comments: Scanned far RAD Properties.

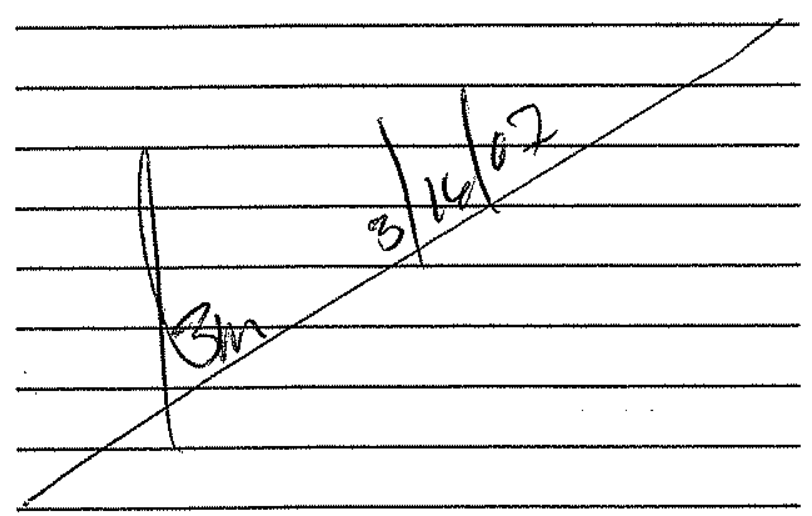

Surveyor: $\quad M B$

\section{Instrument Data}

Mfg. Eberline

Model SAC-4

Serial \# 751

Cal Due 08/07/05

Efficiency 33.4

MDA $20 \mathrm{dpm}$

Mfg. N.E. Tech

Model Electra

Serial \# 1429

Cal Due 08/07/05

Efficiency a $20.4 \quad \beta \quad 30.5$

MDA a $57 \quad \beta \quad 338$
Mfg. Eberline

Model BC-4

Serial \# BC-838

Cal Due 02/07/06

Efficiency 25.6

MDA $346 \mathrm{dpm}$

Mfg. Bicron

Model Micro Rem

Serial \# C3036

Cal Due 08/07/05

Efficiency N/A

MDA $\quad 17 \mu R E M / h r$

SURVEY RESULTS

\begin{tabular}{|c|c|c|c|c|c|c|c|}
\hline & & ALPHA & ALPHA & BETA & BETA & Exposure & Other \\
\hline SWIPE & LOCATION & Swipe & Frisk & Swipe & Frisk & Micro Rem & - \\
\hline$\#$ & & $\mathrm{dpm} / 100 \mathrm{~cm}^{2}$ & $\mathrm{dpm} / 100 \mathrm{~cm}^{2}$ & $\mathrm{dpm} / 100 \mathrm{~cm}^{2}$ & $\mathrm{dpm} / 100 \mathrm{~cm}^{2}$ & $\mu \mathrm{REM} / \mathrm{hr}$ & $=$ \\
\hline 1 & Cooler Exterior & $=$ & $<57$ & - & 2338 & 417 unesinthos & - \\
\hline 2 & Cooler Interior & 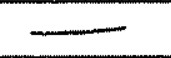 & $<57$ & - & $\angle 338$ & 47 wRen/h & - \\
\hline 3 & Sample Bag \#1 & - & $<57$ & - & $\angle 338$ & Lizulen $1 \mathrm{~h}$ & 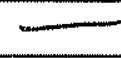 \\
\hline 4 & Sample Bag \#2 & - & $<57$ & - & $<338$ & - & - \\
\hline 5 & Sample Bubble Wrap \# & $\longrightarrow$ & $<57$ & - & $\angle 338$ & - & - \\
\hline 6 & Sample \# 5 & - & $<57$ & - & 4338 & - & - \\
\hline 7 & Sample Bubble Wrap \# & - & - & 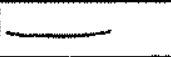 & - & 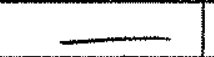 & - \\
\hline 8 & Sample \# 6 & $=$ & $<57$ & 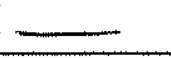 & $<338$ & - & - \\
\hline 9 & Sample Bubble Wrap \# & $\ldots$ & - & $-\quad$ & - & 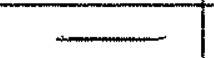 & $=$ \\
\hline 10 & Sample \# 7 & - & $\angle 57$ & - & $\angle 338$ & - & - \\
\hline 11 & Sample Bubble Wrap \# & 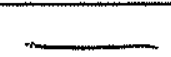 & - & - & - & - & - \\
\hline 12 & Sample \# 8 & 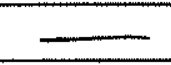 & $\angle 57$ & $=$ & $\angle 336$ & $\square$ & - \\
\hline 13 & Sample Bubble Wrap \# & $\ldots$ & - & 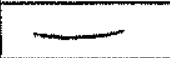 & 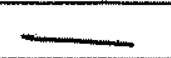 & 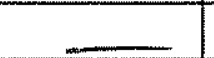 & - \\
\hline 14 & Sample \# & 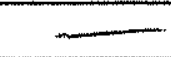 & 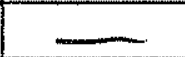 & - & $\longrightarrow$ & - & - \\
\hline 15 & Baxk Gracund & 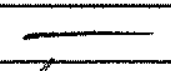 & $<57$ & $\bar{L}$ & $\angle 338$ & $\angle 17$ ureals & 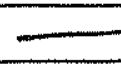 \\
\hline
\end{tabular}

Reviewed by RSO:

Date:

Time: 1720 


\section{CASE NARRATIVE REPORT}

for

Washington Savannah River Site

Subcontract No. AC39040N

WSRC Job \#: 07079

Date: April 12, 2007

AAL WO \#: 11733

Laboratory Identification: Accura Analytical Laboratory, Inc.

\section{Summary:}

Sample Receipt:

Eight solid samples from the Washington Savannah River Site arrived at Accura Analytical Laboratory, Inc. on March 16, 2007 for analysis. The sample listed on the chain arrived to the laboratory via FedEx. A twenty-eight day turnaround was requested on the chain of custody.

The sample was stored properly according to SW-846 procedures and Laboratory Standard Operating Procedures (SOP).

The laboratory received the following samples:

\begin{tabular}{lll} 
Client Sample ID & Laboratory ID & Matrix \\
\hline $07079-4504-\mathrm{DMR}$ & $11733-001$ & Solid \\
$07079-4508-\mathrm{HTF}$ & $11733-002$ & Solid \\
$07079-4531-\mathrm{DMR}$ & $11733-003$ & Solid \\
$07079-4546-\mathrm{HTF}$ & $11733-004$ & Solid \\
$07079-4637-\mathrm{DMR}$ & $11733-005$ & Solid \\
$07079-4649-\mathrm{HTF}$ & $11733-006$ & Solid \\
$07079-4726-\mathrm{DMR}$ & $11733-007$ & Solid \\
$07079-4728-\mathrm{HTF}$ & $11733-008$ & Solid
\end{tabular}

\section{Case Narrative}

Sample analyses were conducted using methodology as outlined in Accura Analytical Laboratory's Standard Operating Procedures. Any technical or administrative problems during analysis, data review, and reduction are written by analytical fraction in the enclosed narratives.

Data Package:

The enclosed data package contains the following sections: Case Narrative, Certificate of 
Analysis, Surrogate information, Quality Control Results, Sample Receipt Checklist, Rad Sample Receipt Checklist (as appropriate), Chain of Custody, and Nonconformance Reports.

The Certificate of Analysis contains the following headings:

Sample ID:

Lab ID:

Matrix:

Date Collected:

Date Received:

Priority:

Collector:
Sample Identification

This is the laboratory identification number

Sample matrix

Date of sample collection

Date of sample receipt by the laboratory

NA

Party responsible for sample collection

The detail on the Certificate includes the following:
Parameter:
Analyte or characteristic tested for in the sample
Qualifier:
Qualifier used for data interpretation
Result:
DL:
Final result for each parameter
RL:
Method Detection Limit (adjusted)
Units:
Reporting Limit (adjusted)
DF:
Analyst:
Date:
Time:
Batch:
Units of final result
Method:
Dilution Factor
Initials of analyst who performed test
Date of analysis
Time of analysis
Analytical batch in which the sample was analyzed
Analytical method used for the analysis of the sample.
Identified on the report numerically with a corresponding table.
Surrogate Recovery:
Provided for organic analysis only. Surrogate compound identified.
Test:
Analytical test associated with surrogate compound.
Percent \%:
Acceptable Limits:
Surrogate percent recovery
Limits established for surrogate recoveries based upon the method requirements.

The QC Summary Report contains the following headings:

Sample ID:

Batch:

Spike Amt:

Sample Result:

Result:
Analyte or characteristic tested for in the QC sample.

Analytical batch in which the QC sample was analyzed

Nominal concentration of the spiking compound

Amount of compound found in the sample associated with the QC sample.

Amount of compound found in the QC sample 
RPD:

Relative percent difference between LCS/LCS dup, MS/MSD, and/or Sample/Sample duplicate

Recovery:

Recovery for the control samples

Reference Range:

Acceptance limits for control samples

Types of QC samples that may be found on the QC Summary Report and/or Certificate of Analysis are:

Blank:

LCS:

LCS dup:

MD:

MS:

MSD:

PS:

PSD:

SDILT:
Results of the blank analysis for the sample batch

Lab control sample

Lab control sample duplicate

Duplicate analysis of sample

Matrix spike

Matrix spike duplicate

Post-Digestion Spike

Post-Digestion Spike Duplicate

Serial Dilution

The following are definitions of reporting limits used at Accura Analytical Laboratory:

DL Detection Limit: The minimum level of an analyte that can be determined (identified not quantified) with $99 \%$ confidence. The values are normally achieved by preparing and analyzing seven aliquots of laboratory water spiked 1 to 5 times the estimated MDL, taking the standard deviation and multiplying it against the one-tailed t-statistic at $99 \%$.

The detection limit is the minimum concentration of a substance that can be identified, measured, and reported with $99 \%$ confidence that the analyte concentration is above zero. It answers the question "Is it present".

QL Quantitation Limit: The lowest concentration that can be reliably achieved within specified limits of precision and accuracy during routine laboratory operating conditions. The QL is generally 5 to 10 times the MDL. However, it may be nominally chosen within these guidelines to simplify data reporting. For many analytes the QL analyte concentration is selected as the slowest non-zero standard in the calibration curve. Sample QL's are highly matrixdependent. Sample specific preparation and dilution factors are applied to these limits when they are reported.

The QL is always $>\mathrm{DL}$

RL Reporting Limit: Same as QL except where driven by contract or client specifications. If the sample specific preparation and dilution factors cause the $\mathrm{QL}$ to be elevated above the RL, then the QL is used as the RL. 


\section{ACCURA ANALYTICAL LABORATORY, INC \\ A Multi-Service Corporation.}

6017 Financial Drive, Norcross, Georgia 30071, 770-449-8800 (ph), 770-449-5477 (fax)

The quantitation limit is the lowest level at which a chemical may be accurately and reproducibly quantitated. It answers the question "How much is present".

Interpretation of RESULT column on the Certificate of Analysis:

If the final concentration in the sample was found to be above the $\mathrm{RL}$, then the value reported is reported without a flag;

If the final concentration in the sample was found to the below the RL but above the DL, then the value reported is flagged with a "J";

If the final concentration in the sample was found to be below the DL, the value reported is flagged with a "U".

If the final concentration in the sample was found in the corresponding method blank, the value reported is flagged with a " $\mathrm{B}$ ".

If the final concentration for an analyte in the sample was found to be exceeding the upper level of the calibration range, the value reported is flagged with a " $E$ ".

If the final concentration for an analyte in the sample is reported from the dilution run, the value reported is flagged with a "D".

A combination of "JD" and "JB" flags are also used with the above definitions.

\section{Quality Control Flags}

Accura Analytical Laboratory maintains acceptance criteria for QC samples through use of statistical process control (SPC). The SPC limits are used to qualify data usability. The flagging criterion identified in WSRC AN98 Format does not necessarily coincide with the laboratory SPC criteria. There may be instances where the Electronic Data Deliverable (EDD) has flagged data based on the AN98 criteria and the lab has not identified the data to be outside of established control limits.

Those instances where the QC has not met laboratory SPC established criteria will be noted in the section case narratives that are included in this package.

This data package, to the best of my knowledge, is in compliance with technical and administrative requirements.

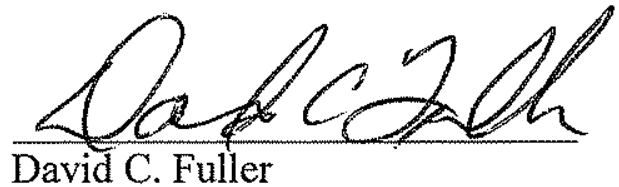

Project Manager - WSRC 


\section{Case Narrative for WSRC \\ Work Order \# 11733 \\ Metals Analysis by ICP \\ Mercury Analysis by CVAA}

Sample Analysis

The following sample was prepared and analyzed for "TCLP Metals", according to the methods referenced in the "Method / Analysis Information" section of this narrative:

\begin{tabular}{ll} 
Client Sample ID & Laboratory ID \\
\hline 07079-4504-DMR & $11733-001$ \\
07079-4508-HTF & $11733-002$ \\
$07079-4531-D M R$ & $11733-003$ \\
$07079-4546-H T F$ & $11733-004$ \\
$07079-4637-D M R$ & $11733-005$ \\
$07079-4649-$ HTF & $11733-006$ \\
$07079-4726-D M R$ & $11733-007$ \\
$07079-4728-H T F$ & $11733-008$
\end{tabular}

TCLP-Metals

\begin{tabular}{ll}
\hline 302349 BLK (Blank) & Method Blank (MB) \\
302349 BKS (Blank Spike) & Laboratory Control Sample (LCS) \\
302349 BSD (Blank Spike Duplicate) & Laboratory Control Sample Duplicate(LCSD) \\
07079-4649-HTF MD & Matrix Sample Duplicate - 11733-006 MD \\
07079-4649-HTF MS & Matrix Spike - 11733-006 MS \\
07079-4649-HTF MSD & Matrix Spike Duplicate - 11733-006 MSD \\
& \\
TCLP-Mercury & \\
\hline 302366 BLK (Blank) & Method Blank (MB) \\
302366 BKS (Blank Spike) & Laboratory Control Sample (LCS) \\
302366 BSD (Blank Spike Duplicate) & Laboratory Control Sample Duplicate(LCSD) \\
07079-4728-HTF MD & Matrix Sample Duplicate - 11733-008 MD \\
07079-4728-HTF MS & Matrix Spike - 11733-008 MS \\
07079-4728-HTF MSD & Matrix Spike Duplicate - 11733-008 MSD
\end{tabular}

Method/Analysis Information:

TCLP Metals

Analysis Batch: $\quad 35559$

Prep Batch \#: $\quad 302349$

Procedure: $\quad$ TCLP-ICP-6010

Analytical Method: SW846 1311/6010

Prep Method: $\quad$ SW846 3050B 
TCLP Mercury

Analysis Batch: $\quad 35601$

Prep Batch \#: $\quad 302366$

Procedure: TCLP-CVAA-7470A

Analytical Method: SW846 1311/7470A

Prep Method: $\quad$ SW846 1311/7470A

\section{System Configuration}

Metals

The analysis was performed on an inductively coupled plasma mass spectrometer. The instrument is equipped with a Meinhardt nebulizer, cyclonic spray chamber, and uses lithium, scandium, yttritium, indium and bismuth as internal standards. Operating conditions for the ICP/MS are set at a power level of 1000 watts. The instrument has a peristaltic pump flow rate of $24 \mathrm{RPM}(2.0 \mathrm{~mL} / \mathrm{min}$ sample uptake rate), argon gas flow of $15 \mathrm{~L} / \mathrm{min}$ for the torch and $0.5 \mathrm{~L} / \mathrm{min}$ for the plasma (torch tip), and a pressure setting of 20 PSI for the nebulizer.

Mercury

Mercury analysis was performed on a Flow Injection Mercury System (FIMS-400) automated mercury analyzer. The instrument consists of a cold vapor atomic absorption spectrometer set to detect mercury at a wavelength of $254 \mathrm{~nm}$. Sample introduction through the flow injection system is performed via a peristaltic pump at $9-\mathrm{mL} / \mathrm{min}$ and nitrogen carrier gas rate of $5 \mathrm{~L} / \mathrm{min}$.

\section{Sample Preparation}

The samples were prepared in accordance with the referenced SW-846 procedures.

\section{Calibration Information}

\section{Initial Calibration}

Instrument calibrations were conducted using method and instrument manufacturer's specification. All initial calibration requirements have been met for this analysis.

\section{CRDL Standard}

All CRDL standard recoveries met the advisory limits.

\section{ICSA/ICSAB Requirements}

All interference check samples (ICSA and ICSAB) associated with this work order met the established acceptance criteria.

\section{Continuing Calibration Blanks (CCB) Requirements}

All continuing calibration blanks (CCB) bracketing sample analyses associated with this work order met the established acceptance criteria. 


\section{Continuing Calibration Verification (CCV) Requirements}

All continuing calibration verification (CCV) standards bracketing sample analyses associated with this work order met the recovery acceptance criteria.

\section{Quality Control (QC) Information}

\section{Method Blank Acceptance}

The method blanks analyzed with this job did not contain analytes of interest at concentrations greater than the reporting limit (RL).

\section{LCS/LCSD Recovery Statement}

All LCS and LCSD spike recoveries for this job were within the required acceptance limits.

\section{QC Sample Designation}

Sample 07079-4649-HTF from WSRC Job 07079 was designated as the quality control sample for the TCLP Metals ICP batch and sample 07079-4728-HTF was designated QC sample for the TCLP Mercury CVAA batch.

The ICP and the CVAA batch included a Laboratory Control Sample (LCS), Laboratory Control Sample Duplicate(LCSD), Matrix Spike (MS) and Matrix Spike Duplicate (MSD).

\section{MS/MSD Recovery Statement}

The percent recoveries (\%R) obtained from the matrix spike (MS) and matrix spike duplicate (MSD) analyses were evaluated when the sample concentration was less than four times (4X) the spike concentration added. All applicable elements in the MS and MSD analyses met the established recovery limits.

\section{MS/MSD RPD Statement}

The relative percent difference (RPD) between the MS and MSD recoveries were within the acceptance limits for all the analytes for this work order.

\section{Serial Dilution \% Difference Statement}

The serial dilution is used to access interference caused by matrix suppression or enhancement. Raw element concentrations that are at least 50X the instrument detection limit (IDL) for ICP analyses are applicable for serial dilution assessment.

All applicable elements met the established criteria for serial dilution evaluations, percent differences values $<10$.

\section{Technical Information}

\section{Holding Time Specification}

The samples associated with this work order met the specified holding time requirements. 


\section{Sample Dilution}

Dilutions are performed to minimize matrix interference resulting from elevated mineral elements concentrations present in samples and/or to bring over range target analyte concentrations into the linear calibration range of this instruments. No sample dilution was required in this work order:

\section{LQCF $(N C R)$ Documentation}

Laboratory Quality Communication Forms (LQCFs) are generated to document procedural anomalies that may deviate from referenced SOPs or Contractual documents. No LQCF was necessary for this work order.

\section{Additional Comments}

The additional comments field is used to address special issues associated with each analysis, clarify method/contractual issues pertaining to the analysis and to list any report documents generated as a result of sample analysis or review. No additional comments were required for this work order.

\section{Review / Validation}

The laboratory requires all analytical data to be verified by a qualified data validator.

The following data validator verified the data presented in this job.

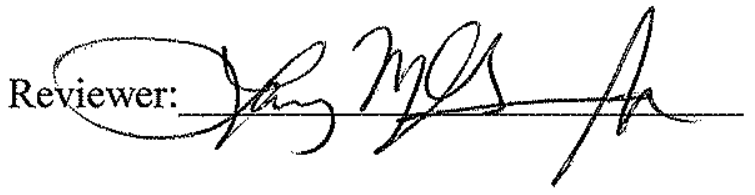

Date: $\quad$ April 13,2007 
Certificate of Analytical Results 11733

Washington Savannah River Co., Aiken, SC

07079

Sample Id: 07079-4504-DMR

Lab Sample Id: 11733-001

Sample Depth:

Matrix: SOLID

Date Collected: Mar-15-07 09:05
$\%$ Moisture:

Date Received: Mar-16-07 10:11
Analytical Method: TCLP Mercury by SW1311/7470A

Date Analyzed: Apr $03-07$ 16:39

Analyst: MSN01

Seq Number: 35601
Prep Method: SW7470A_DIG

Date Prep: Apr-02-07 15:00 Tech: MSN01

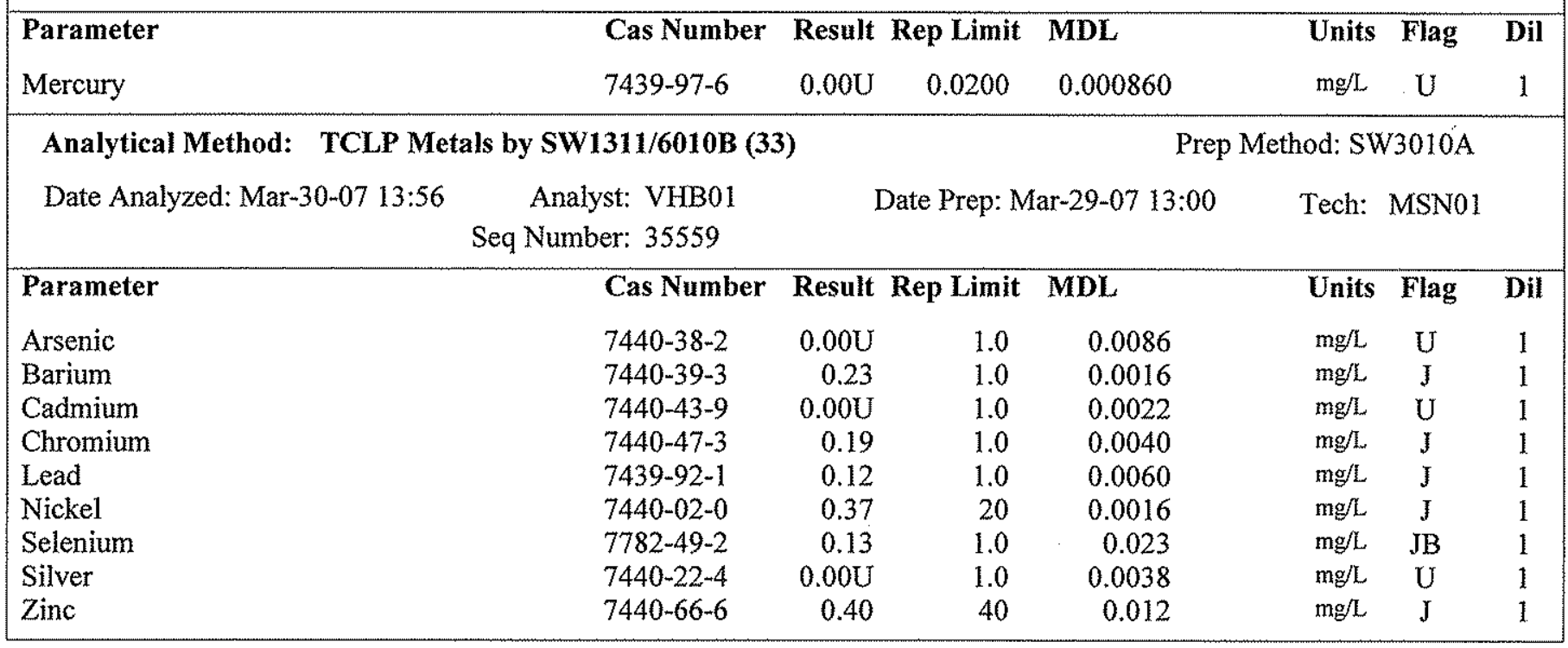


Certificate of Analytical Results 11733

Washington Savannah River Co., Aiken, SC

07079

Sample Id: 07079-4508-HTF

Lab Sample Id: 11733-002

Sample Depth:
Matrix: SOLID

Date Collected: Mar-15-07 09:06
\% Moisture:

Date Received: Mar-16-07 10:11

Analytical Method: TCLP Mercury by SW1311/7470A

Prep Method: SW7470A DIG

Date Analyzed: Apr-03-07 16:43

Analyst: MSN01

Date Prep: Apr-02-07 15:00

Tech: MSNO1

Seq Number: 35601

$\begin{array}{lccccccc}\text { Parameter } & \text { Cas Number } & \text { Result Rep Limit } & \text { MDL } & & \text { Units } & \text { Flag } & \text { Dil } \\ \text { Mercury } & 7439-97-6 & 0.00 \mathrm{U} & 0.0200 & 0.000860 & \mathrm{nig} / \mathrm{L} & \mathrm{U} & 1\end{array}$

Analytical Method: TCLP Metals by SW1311/6010B (33)

Prep Method: SW3010A

Date Analyzed: Mar-30-07 14:02 Analyst: VHB01

Date Prep: Mar-29-07 13:00 Tech: MSN01

Seq Number: 35559

\begin{tabular}{|c|c|c|c|c|c|c|c|}
\hline Parameter & Cas Number & Result & Rep Limit & IDL & Units & Flag & Dil \\
\hline Arsenic & $7440-38-2$ & $0.00 \mathrm{U}$ & 1.0 & 0.0086 & $\mathrm{mg} / \mathrm{L}$ & $\mathrm{U}$ & 1 \\
\hline Barium & $7440-39-3$ & 0.29 & 1.0 & 0.0016 & $\mathrm{mg} / \mathrm{L}$ & J & 1 \\
\hline Cadmium & $7440-43-9$ & $0.00 \mathrm{U}$ & 1.0 & 0.0022 & $\mathrm{mg} / \mathrm{L}$ & $U$ & 1 \\
\hline Chromium & $7440-47-3$ & 0.11 & 1.0 & 0.0040 & $\mathrm{mg} / \mathrm{L}$ & J & 1 \\
\hline Lead & $7439-92-1$ & 0.020 & 1.0 & 0.0060 & $\mathrm{mg} / \mathrm{L}$ & J & 1 \\
\hline Nickel & $7440-02-0$ & 0.0044 & 20 & 0.0016 & $\mathrm{mg} / \mathrm{L}$ & J & 1 \\
\hline Selenium & $7782-49-2$ & 0.13 & 1.0 & 0.023 & $\mathrm{mg} / \mathrm{L}$ & JB & 1 \\
\hline Silver & $7440-22-4$ & $0.00 \mathrm{U}$ & 1.0 & 0.0038 & $\mathrm{mg} / \mathrm{L}$ & U & 1 \\
\hline Zinc & $7440-66-6$ & 0.018 & 40 & 0.012 & $\mathrm{mg} / \mathrm{L}$ & $\mathrm{JB}$ & 1 \\
\hline
\end{tabular}


Certificate of Analytical Results 11733

Washington Savannah River Co, Aiken, SC

07079

Sample Id: 07079-4531-DMR

Lab Sample Id: 11733-003

Sample Depth:
Matrix: SOLID

Date Collected: Mar-15-07 09:07
$\%$ Moisture:

Date Received: Mar-16-07 10:11

Analytical Method: TCLP Mercury by SW1311/7470A

Prep Method: SW7470A DIG

Date Analyzed: Apr-03-07 16:47

Analyst: MSN01

Date Prep: Apr-02-07 15:00

Tech: MSN01

Seq Number: 35601

\begin{tabular}{|lccccccc}
\hline Parameter & Cas Number & Result Rep Limit & MDL & & Units & Flag & Dil \\
Mercury & $7439-97-6$ & $0.00 \mathrm{U}$ & 0.0200 & 0.000860 & $\mathrm{mg} / \mathrm{L}$ & $\mathrm{U}$ & 1
\end{tabular}

Analytical Method: TCLP Metals by SW1311/6010B (33)

Prep Method: SW3010A

Date Analyzed: Mar-30-07 15:13 Analyst: VHB01

Seq Number: 35559

\begin{tabular}{|lrrrrccc}
\hline Parameter & Cas Number & Result Rep Limit & MDL & & Units & Flag & Dil \\
Arsenic & $7440-38-2$ & $0.00 \mathrm{U}$ & 1.0 & 0.0086 & $\mathrm{mg} / \mathrm{L}$ & $\mathrm{U}$ & 1 \\
Barium & $7440-39-3$ & 0.20 & 1.0 & 0.0016 & $\mathrm{mg} / \mathrm{L}$ & $\mathrm{J}$ & 1 \\
Cadmium & $7440-43-9$ & $0.00 \mathrm{U}$ & 1.0 & 0.0022 & $\mathrm{mg} / \mathrm{L}$ & $\mathrm{U}$ & 1 \\
Chromium & $7440-47-3$ & 0.15 & 1.0 & 0.0040 & $\mathrm{mg} / \mathrm{L}$ & $\mathrm{J}$ & 1 \\
Lead & $7439-92-1$ & 0.074 & 1.0 & 0.0060 & $\mathrm{mg} / \mathrm{L}$ & $\mathrm{J}$ & 1 \\
Nickel & $7440-02-0$ & 0.34 & 20 & 0.0016 & $\mathrm{mg} / \mathrm{L}$ & $\mathrm{J}$ & 1 \\
Selenium & $7782-49-2$ & 0.13 & 1.0 & 0.023 & $\mathrm{mg} / \mathrm{L}$ & $\mathrm{JB}$ & 1 \\
Silver & $7440-22-4$ & $0.00 \mathrm{U}$ & 1.0 & 0.0038 & $\mathrm{mg} / \mathrm{L}$ & $\mathrm{U}$ & 1 \\
Zinc & $7440-66-6$ & 0.57 & 40 & 0.012 & $\mathrm{mg} / \mathrm{L}$ & $\mathrm{J}$ & 1 \\
\hline
\end{tabular}

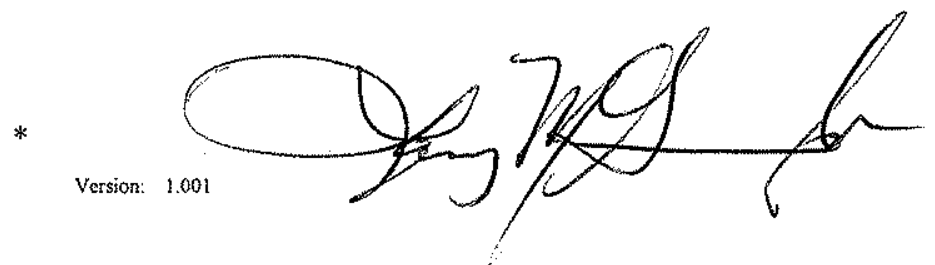




\section{Certificate of Analytical Results 11733}

Washington Savannah River Co., Aiken, SC

07079

Sample Id: 07079-4546-HTF

Lab Sample Id: 11733-004

Sample Depth:

\section{Matrix: SOLID}

Date Collected: Mar-15-07 09:08
$\%$ Moisture:

Date Received: Mar-16-07 10:11

Analytical Method: TCLP Mercury by SW1311/7470A

Prep Method: SW7470A_DIG

Date Analyzed: Apr-03-07 16:51

Analyst: MSN01

Date Prep: Apr-02-07 15:00

Tech: MSN01

Seq Number: 35601

\begin{tabular}{|lccccccc|}
\hline Parameter & Cas Number & Result Rep Limit & MDL & Units & Flag & Dil \\
Mercury & $7439-97-6$ & $0.00 \mathrm{U}$ & 0.0200 & 0.000860 & $\mathrm{mg} / \mathrm{L}$ & $\mathrm{U}$ & 1 \\
\hline
\end{tabular}

Analytical Method: TCLP Metals by SW1311/6010B (33)

Prep Method: SW3010A

Date Analyzed: Mar-30-07 15:18 Analyst: VHB01 Seq Number: 35559

\begin{tabular}{|lcrrrrcc|}
\multicolumn{7}{c}{ Seq Number: 35559} \\
\hline Parameter & Cas Number & Result Rep Limit & MDL & Units & Flag & Dil \\
Arsenic & $7440-38-2$ & $0.00 \mathrm{U}$ & 1.0 & 0.0086 & $\mathrm{mg} / \mathrm{L}$ & $\mathrm{U}$ & 1 \\
Barium & $7440-39-3$ & 0.19 & 1.0 & 0.0016 & $\mathrm{~m} / \mathrm{L}$ & $\mathrm{J}$ & 1 \\
Cadmium & $7440-43-9$ & $0.00 \mathrm{U}$ & 1.0 & 0.0022 & $\mathrm{mg} / \mathrm{L}$ & $\mathrm{U}$ & 1 \\
Chromium & $7440-47-3$ & 0.016 & 1.0 & 0.0040 & $\mathrm{mg} / \mathrm{L}$ & $\mathrm{J}$ & 1 \\
Lead & $7439-92-1$ & 0.011 & 1.0 & 0.0060 & $\mathrm{~m} / \mathrm{L}$ & $\mathrm{J}$ & 1 \\
Nickel & $7440-02-0$ & 0.010 & 20 & 0.0016 & $\mathrm{~m} / \mathrm{L}$ & $\mathrm{J}$ & 1 \\
Selenium & $7782-49-2$ & 0.12 & 1.0 & 0.023 & $\mathrm{~m} / \mathrm{L}$ & $\mathrm{JB}$ & 1 \\
Silver & $7440-22-4$ & $0.00 \mathrm{U}$ & 1.0 & 0.0038 & $\mathrm{mg} / \mathrm{L}$ & $\mathrm{U}$ & 1 \\
Zinc & $7440-66-6$ & 0.017 & 40 & 0.012 & $\mathrm{mg} / \mathrm{L}$ & $\mathrm{JB}$ & 1 \\
\hline
\end{tabular}

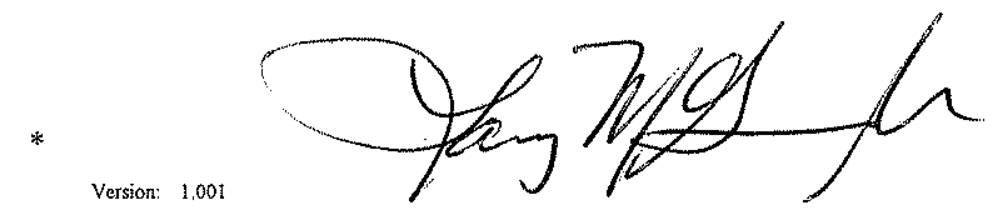

Date Prep: Mar-29-07 13:00 Tech: MSN01 
Certificate of Analytical Results 11733

Washington Savannah River Co., Aiken, SC

07079

Sample Id: 07079-4637-DMR

Lab Sample Id: 11733-005

Sample Depth:
$\%$ Moisture:

Date Received: Mar-16-07 10:11
Analytical Method: TCLP Mercury by SW1311/7470A

Date Analyzed: Apr-03-07 16:55

Analyst: MSN01

Seq Number: 35601

Matrix: SOLID

Date Collected: Mar-15-07 09:09

Prep Method: SW7470A_DIG

Date Prep: Apr-02-07 15:00

Tech: MSN01

Cas Number Result Rep Limit MDL

Units Flag DiI

Mercury

7439-97-6

$0.00 \mathrm{U}$

0.0200

0.000860

$\mathrm{mg} / \mathrm{L} \quad \mathrm{U}$

Prep Method: SW3010A

Analytical Method: TCLP Metals by SW1311/6010B (33)

Date Analyzed: Mar-30-07 15:23 Analyst: VHB01

Seq Number: 35559

\begin{tabular}{|c|c|c|c|c|c|c|c|}
\hline Date Analyzed: Mar-30-07 15:23 & $\begin{array}{l}\text { Analyst: VHB01 } \\
\text { Seq Number: } 35559\end{array}$ & & Date Prep: $N$ & ar-29-07 13:00 & Tech: & MSNO & \\
\hline Parameter & Cas Number & Result & Rep Limit & MDL & Units & Flag & Dil \\
\hline Arsenic & $7440-38-2$ & 0.011 & 1.0 & 0.0086 & $\mathrm{mg} / \mathrm{L}$ & J & 1 \\
\hline Barium & $7440-39-3$ & 0.29 & 1.0 & 0.0016 & $\mathrm{mg} / \mathrm{L}$ & J & 1 \\
\hline Cadmium & $7440-43-9$ & $0.00 \mathrm{U}$ & 1.0 & 0.0022 & $\mathrm{mg} / \mathrm{L}$ & U & 1 \\
\hline Chromium & $7440-47-3$ & 0.44 & 1.0 & 0.0040 & $\mathrm{mg} / \mathrm{L}$ & $J$ & 1 \\
\hline Lead & $7439-92-1$ & 0.20 & 1.0 & 0.0060 & $\mathrm{mg} / \mathrm{L}$ & $\mathrm{J}$ & 1 \\
\hline Nickel & $7440-02-0$ & 0.37 & 20 & 0.0016 & $\mathrm{mg} / \mathrm{L}$ & J & 1 \\
\hline Selenium & $7782-49-2$ & 0.12 & 1.0 & 0.023 & $\mathrm{mg} / \mathrm{L}$ & $\mathrm{JB}$ & 1 \\
\hline Silver & $7440-22-4$ & $0.00 \mathrm{U}$ & 1.0 & 0.0038 & $\mathrm{mg} / \mathrm{L}$ & U & 1 \\
\hline Zinc & $7440-66-6$ & 0.76 & 40 & 0.012 & $\mathrm{mg} / \mathrm{L}$ & $\mathrm{J}$ & 1 \\
\hline
\end{tabular}

Tech: MSN01 


\section{Certificate of Analytical Results 11733}

Washington Savannah River Co., Aiken, SC

07079

Sample Id: 07079-4649-HTF

Lab Sample Id: 11733-006

Sample Depth:

\section{Matrix: SOLID}

Date Collected: Mar-15-07 09:10
$\%$ Moisture:

Date Received: Mar-16-07 10:11

Analytical Method: TCLP Mercury by SW1311/7470A

Prep Method: SW7470A_DIG

Date Analyzed: Apr-03-07 17:06

Analyst: MSN01

Date Prep: Apr-02-07 15:00

Tech: MSN01

Seq Number: 35601

\begin{tabular}{llcccccc}
\hline Parameter & Cas Number & Result Rep Limit & MDL & Units & Flag & Dil \\
Mercury & $7439-97-6$ & $0.00 \mathrm{U}$ & 0.0200 & 0.000860 & $\mathrm{mg} / \mathrm{L}$ & $\mathrm{U}$ & 1
\end{tabular}

Analytical Method: TCLP Metals by SW1311/6010B (33)

Prep Method: SW3010A

Date Analyzed: Mar-30-07 13:31 Analyst: VHB01

Date Prep: Mar-29-07 13:00 Tech: MSN01 Seq Number: 35559

\begin{tabular}{llrrrccc|}
\hline Parameter & Cas Number & Result Rep Limit & MDL & Units & Flag & Dil \\
Arsenic & $7440-38-2$ & $0.00 \mathrm{U}$ & 1.0 & 0.0086 & $\mathrm{mg} / \mathrm{L}$ & $\mathrm{U}$ & 1 \\
Barium & $7440-39-3$ & 0.34 & 1.0 & 0.0016 & $\mathrm{mg} / \mathrm{L}$ & $\mathrm{J}$ & 1 \\
Cadmium & $7440-43-9$ & $0.00 \mathrm{U}$ & 1.0 & 0.0022 & $\mathrm{mg} / \mathrm{L}$ & $\mathrm{U}$ & 1 \\
Chromium & $7440-47-3$ & 0.012 & 1.0 & 0.0040 & $\mathrm{~m} / \mathrm{L}$ & $\mathrm{J}$ & 1 \\
Lead & $7439-92-1$ & 0.013 & 1.0 & 0.0060 & $\mathrm{~m} / \mathrm{L}$ & $\mathrm{J}$ & 1 \\
Nickel & $7440-02-0$ & 0.0050 & 20 & 0.0016 & $\mathrm{mg} / \mathrm{J}$ & $\mathrm{J}$ & 1 \\
Selenium & $7782-49-2$ & 0.11 & 1.0 & 0.023 & $\mathrm{mg} / \mathrm{L}$ & $\mathrm{JB}$ & 1 \\
Silver & $7440-22-4$ & $0.00 \mathrm{U}$ & 1.0 & 0.0038 & $\mathrm{mg} / \mathrm{L}$ & $\mathrm{U}$ & 1 \\
Zinc & $7440-66-6$ & 0.019 & 40 & 0.012 & $\mathrm{mg} / \mathrm{J}$ & $\mathrm{JB}$ & 1 \\
\hline
\end{tabular}




\section{Certificate of Analytical Results 11733}

\section{Washington Savannah River Co., Aiken, SC}

07079

Sample Id: 07079-4726-DMR

Lab Sample Id: 11733-007

Sample Depth:

\section{Matrix: SOLID}

Date Collected: Mar-15-07 09:11
$\%$ Moisture:

Date Received: Mar-16-07 10:11

Analytical Method: TCLP Mercury by SW1311/7470A

Prep Method: SW7470A_DIG

Date Analyzed: Apr-03-07 17:10

Analyst: MSN01

Date Prep: Apr-02-07 15:00

Tech: MSN01

Seq Number: 35601

\begin{tabular}{lllllccc}
\hline Parameter & Cas Number & Result & Rep Limit & MDL & Units & Flag & Dil \\
Mercury & $7439-97-6$ & $0.00 \mathrm{U}$ & 0.0200 & 0.000860 & $\mathrm{mg} / \mathrm{L}$ & $\mathrm{U}$ & 1
\end{tabular}

Analytical Method: TCLP Metals by SW1311/6010B (33)

Prep Method: SW3010A

Date Analyzed: Mar-30-07 15:28 Analyst: VHB01 Seq Number: 35559

\begin{tabular}{|lcrrrccc|}
\multicolumn{7}{c}{ Seq Number: 35559} \\
\hline Parameter & Cas Number & Result Rep Limit & MDL & Units & Flag & Dil \\
Arsenic & $7440-38-2$ & 0.014 & 1.0 & 0.0086 & $\mathrm{mg} / \mathrm{L}$ & $\mathrm{J}$ & 1 \\
Barium & $7440-39-3$ & 0.27 & 1.0 & 0.0016 & $\mathrm{mg} / \mathrm{L}$ & $\mathrm{J}$ & 1 \\
Cadmium & $7440-43-9$ & $0.00 \mathrm{U}$ & 1.0 & 0.0022 & $\mathrm{mg} / \mathrm{L}$ & $\mathrm{U}$ & 1 \\
Chromium & $7440-47-3$ & 0.055 & 1.0 & 0.0040 & $\mathrm{mg} / \mathrm{L}$ & $\mathrm{J}$ & 1 \\
Lead & $7439-92-1$ & 0.024 & 1.0 & 0.0060 & $\mathrm{mg} / \mathrm{L}$ & $\mathrm{J}$ & 1 \\
Nickel & $7440-02-0$ & 0.046 & 20 & 0.0016 & $\mathrm{mg} / \mathrm{L}$ & $\mathrm{J}$ & 1 \\
Selenium & $7782-49-2$ & 0.10 & 1.0 & 0.023 & $\mathrm{mg} / \mathrm{L}$ & $\mathrm{JB}$ & 1 \\
Silver & $7440-22-4$ & $0.00 \mathrm{U}$ & 1.0 & 0.0038 & $\mathrm{mg} / \mathrm{L}$ & $\mathrm{U}$ & 1 \\
Zinc & $7440-66-6$ & 0.10 & 40 & 0.012 & $\mathrm{mg} / \mathrm{L}$ & $\mathrm{JB}$ & 1 \\
\hline
\end{tabular}

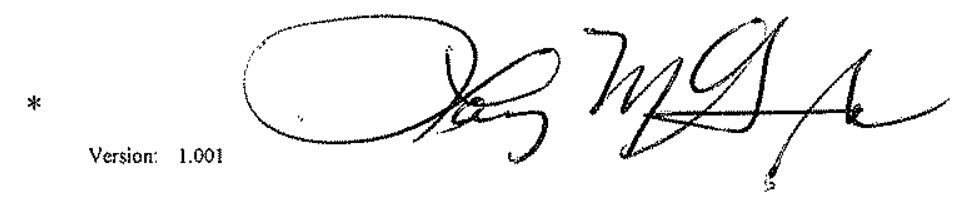




\section{Certificate of Analytical Results 11733}

Washington Savannah River Co., Aiken, SC

07079

Sample Id: 07079-4728-HTF

Lab Sample Id: 11733-008

Sample Depth:

\section{Matrix: SOLID}

Date Collected: Mar-15-07 09:12
$\%$ Moisture:

Date Received: Mar-16-07 10:11

Analytical Method: TCLP Mercury by SW1311/7470A

Prep Method: SW7470A_DIG

Date Analyzed: Apr-03-07 16:24

Analyst: MSN01

Date Prep: Apr-02-07 15:00

Tech: MSN01

Seq Number: 35601

\begin{tabular}{|lccccccc|}
\hline Parameter & Cas Number & Result & Rep Limit & MDL & Units & Flag & Dil \\
Mercury & $7439-97-6$ & $0.00 \mathrm{U}$ & 0.0200 & 0.000860 & $\mathrm{mg} / \mathrm{L}$ & $\mathrm{U}$ & 1
\end{tabular}

Analytical Method: TCLP Metals by SW1311/6010B (33)

Prep Method: SW3010A

Date Analyzed: Mar-30-07 15:33 Analyst: VHB01

Seq Number: 35559

\begin{tabular}{llrrrccc|}
\hline Parameter & Cas Number & Result & Rep Limit & MDL & Units & Flag & Dil \\
Arsenic & $7440-38-2$ & $0.00 \mathrm{U}$ & 1.0 & 0.0086 & $\mathrm{mg} / \mathrm{L}$ & $\mathrm{U}$ & 1 \\
Barium & $7440-39-3$ & 0.26 & 1.0 & 0.0016 & $\mathrm{mg} / \mathrm{L}$ & $\mathrm{J}$ & 1 \\
Cadmium & $7440-43-9$ & $0.00 \mathrm{U}$ & 1.0 & 0.0022 & $\mathrm{mg} / \mathrm{L}$ & $\mathrm{U}$ & 1 \\
Chromium & $7440-47-3$ & $0.00 \mathrm{U}$ & 1.0 & 0.0040 & $\mathrm{mg} / \mathrm{L}$ & $\mathrm{U}$ & 1 \\
Lead & $7439-92-1$ & 0.016 & 1.0 & 0.0060 & $\mathrm{mg} / \mathrm{L}$ & $\mathrm{J}$ & 1 \\
Nickel & $7440-02-0$ & $0.00 \mathrm{U}$ & 20 & 0.0016 & $\mathrm{mg} / \mathrm{L}$ & $\mathrm{U}$ & 1 \\
Selenium & $7782-49-2$ & 0.14 & 1.0 & 0.023 & $\mathrm{mg} / \mathrm{L}$ & $\mathrm{JB}$ & 1 \\
Silver & $7440-22-4$ & $0.00 \mathrm{U}$ & 1.0 & 0.0038 & $\mathrm{mg} / \mathrm{L}$ & $\mathrm{U}$ & 1 \\
Zinc & $7440-66-6$ & $0.00 \mathrm{U}$ & 40 & 0.012 & $\mathrm{mg} / \mathrm{L}$ & $\mathrm{U}$ & 1 \\
\hline
\end{tabular}




\section{Sample Duplicate Recovery}

\section{Project Name: 07079}

Work Order \#: 11733

Report Date: 04/12/07 12:44

Lab Batch \#: 35601

QC-Sample ID: $11733-008 \mathrm{MD}$

Reporting Units: $\mathrm{mg} / \mathrm{L}$

Project ID: 07079

\begin{tabular}{|c|c|c|c|c|c|}
\hline TCLP Mercury by SW1311/7470A & $\begin{array}{c}\text { Parent Sample } \\
\text { Result } \\
{[\mathrm{A}]}\end{array}$ & $\begin{array}{c}\text { Sample } \\
\text { Duplicate } \\
\text { Result } \\
{[\mathrm{B}]}\end{array}$ & RPD & $\begin{array}{c}\text { Control } \\
\text { Limits } \\
\% \text { RPD }\end{array}$ & Flag \\
\hline Analyte & 0.000 & 0.000 & $\mathrm{NC}$ & 20 & \\
\hline
\end{tabular}

Lab Batch \#: 35559

QC-Sample ID: 11733-006 MD

Reporting Units: $\mathrm{mg} / \mathrm{L}$

Batch \#: $1 \quad$ Matrix: Sd

\begin{tabular}{|c|c|c|c|c|c|}
\hline $\begin{array}{c}\text { TCLP Metals by SW1311/6010B } \\
\text { Analyte }\end{array}$ & $\begin{array}{c}\text { Parent Sample } \\
\text { Result } \\
\text { [A] }\end{array}$ & $\begin{array}{l}\text { Sample } \\
\text { Duplicate } \\
\text { Result } \\
\text { [B] }\end{array}$ & RPD & $\begin{array}{l}\text { Control } \\
\text { Limits } \\
\% \text { RPD }\end{array}$ & Flag \\
\hline Arsenic & 0.000 & 0.000 & $\mathrm{NC}$ & 20 & \\
\hline Barium & 0.34 & 0.34 & 1 & 20 & \\
\hline Cadmium & 0.000 & 0.000 & $\mathrm{NC}$ & 20 & \\
\hline Chromium & 0.012 & 0.013 & 8 & 20 & \\
\hline Lead & 0.013 & 0.015 & 14 & 20 & \\
\hline Nickel & 0.0050 & 0.0042 & 17 & 20 & \\
\hline Selenium & 0.11 & 0.11 & 4 & 20 & \\
\hline Silver & 0.000 & 0,000 & $\mathrm{NC}$ & 20 & \\
\hline Zinc & 0.019 & 0.018 & 7 & 20 & \\
\hline
\end{tabular}




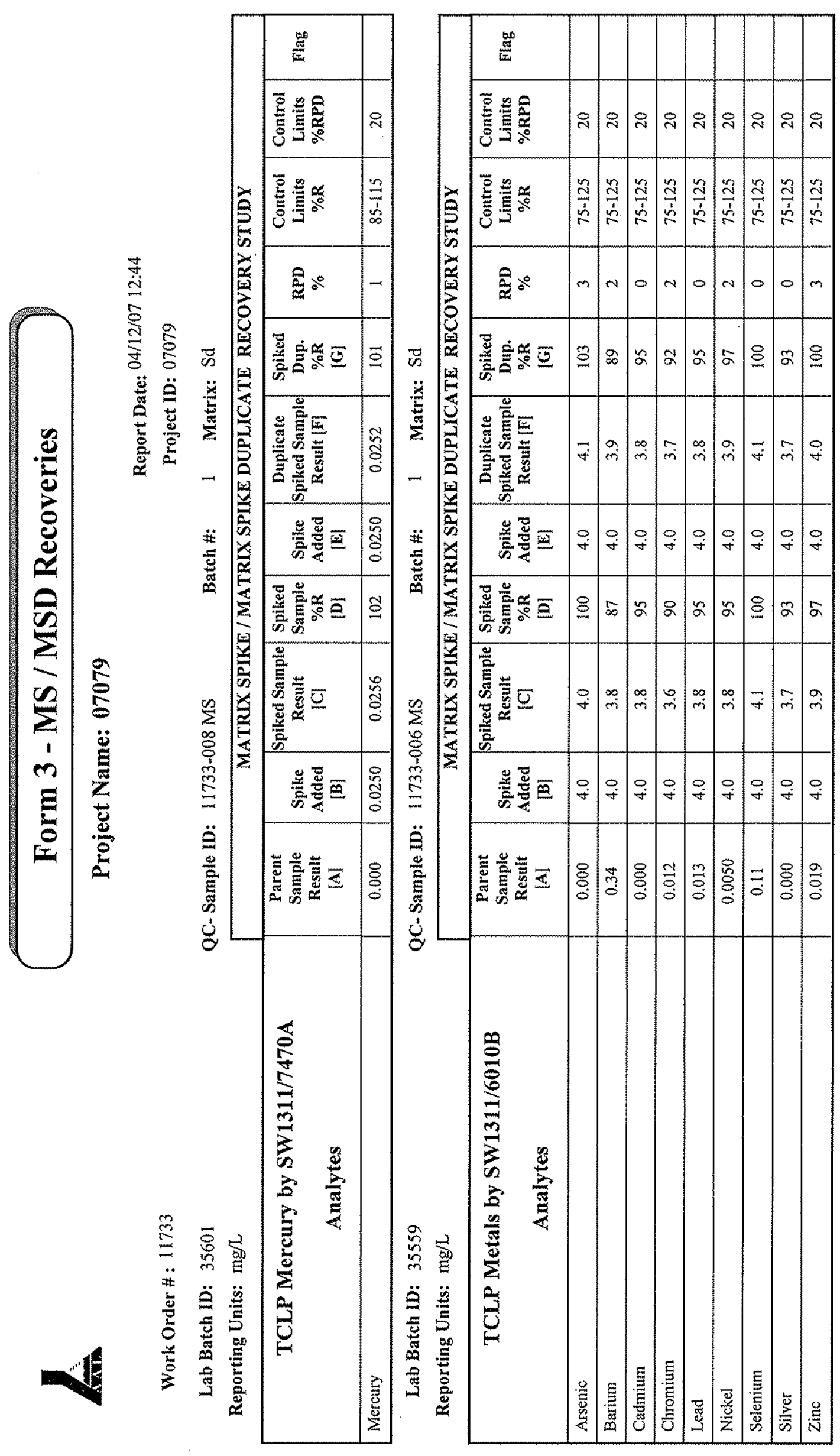

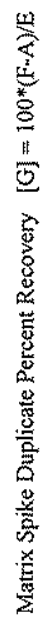

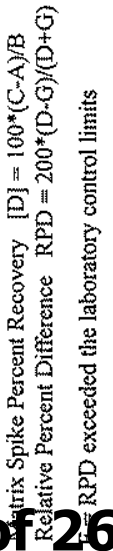



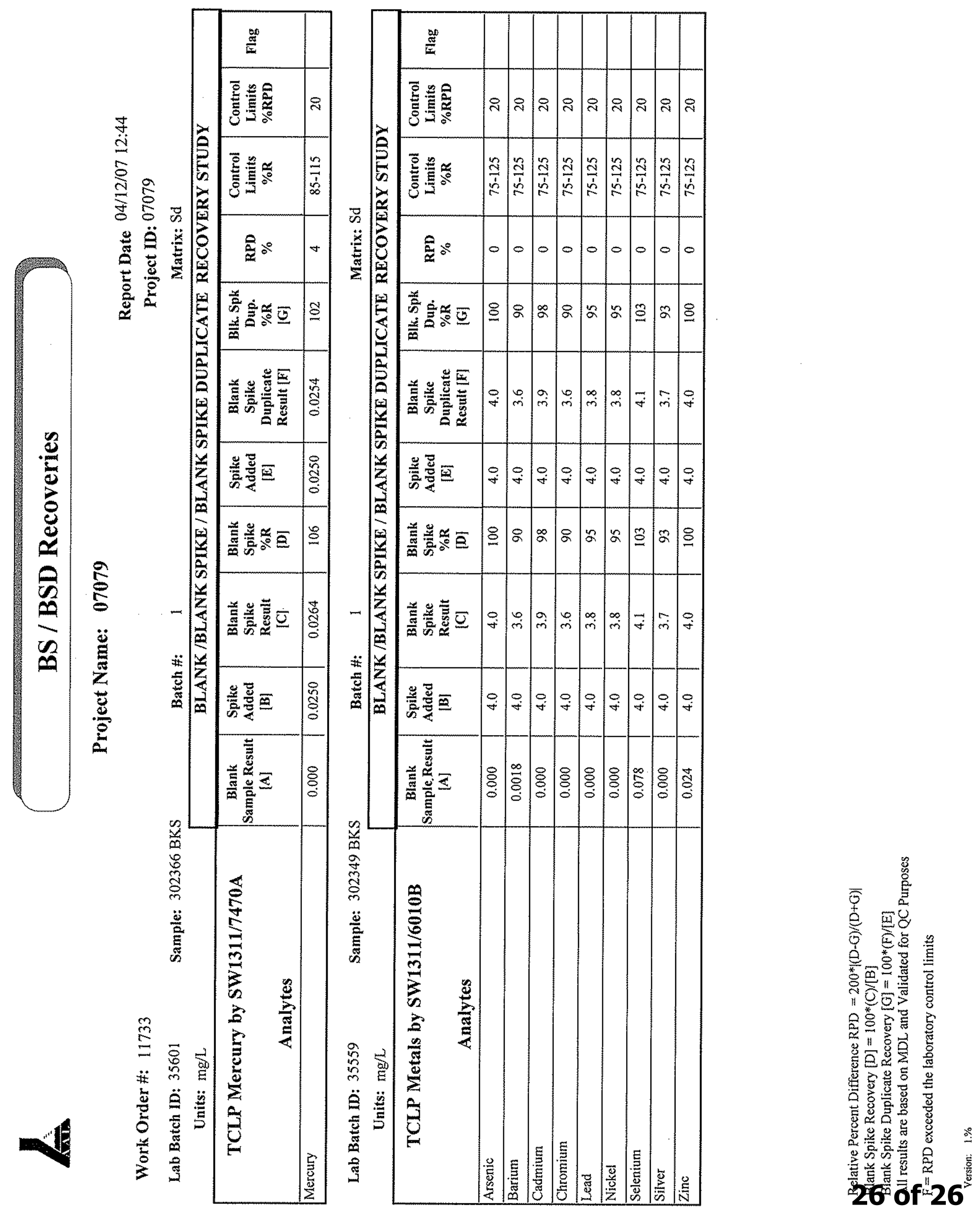